\title{
SPOONS: NETFLIX OUTAGE DETECTION USING MICROTEXT CLASSIFICATION
}

\author{
A Thesis \\ Presented to \\ the Faculty of California Polytechnic State University \\ San Luis Obispo
}

\author{
In Partial Fulfillment \\ of the Requirements for the Degree \\ Master of Science in Computer Science
}

by

Eriq Augustine

March 2013 
(C) 2013

Eriq Augustine

ALL RIGHTS RESERVED 
COMMITTEE MEMBERSHIP

TITLE:

SPOONS: Netflix Outage Detection Using

Microtext Classification

AUTHOR: $\quad$ Eriq Augustine

DATE SUBMITTED: $\quad$ March 2013

COMMITTEE CHAIR: Professor Alex Dekhtyar, Ph.D.

Department of Computer Science

COMmitTeE Member: Professor Clint Staley, Ph.D.

Department of Computer Science

COMMiTTEe MEMBER: Professor Franz Kurfess, Ph.D.

Department of Computer Science

COMMITTEE MEMBER: Assistant Professor Foaad Khosmood, Ph.D. Department of Computer Science 


\begin{abstract}
SPOONS: Netflix Outage Detection Using Microtext Classification
\end{abstract}

\title{
Eriq Augustine
}

Every week there are over a billion new posts to Twitter services and many of those messages contain feedback to companies about their services. One company that recognizes this unused source of information is Netflix. That is why Netflix initiated the development of a system that lets them respond to the millions of Twitter and Netflix users that are acting as sensors and reporting all types of user visible outages. This system enhances the feedback loop between Netflix and its customers by increasing the amount of customer feedback that Netflix receives

and reducing the time it takes for Netflix to receive the reports and respond to them.

The goal of the SPOONS (Swift Perceptions of Online Negative Situations) system is to use Twitter posts to determine when Netflix users are reporting a problem with any of the Netflix services. This work covers the architecture of the SPOONS system and framework as well as outage detection using tweet classification. 


\section{Acknowledgements}

Thanks to:

- Alex, ABRA, Netflix especially Kevin McEntee, and all the funions.

- The Cal Poly Computer Science Department for being the supportive and helpful.

- All of the department office staff: Cindy, Christy, and Susanne.

- My family for raising me, especially my parents (they are pretty cool).

- My roommates for providing support and unity.

- Farscape for encouraging team bonding and providing a common enemy.

- VIM for being the best text editor. 


\section{Contents}

List of Tables $\quad$ xi

List of Figures $\quad$ xv

1 Introduction 1

1 Problem: Swift Perception Of Online Negative Situations 2

$\begin{array}{lll}2 & \text { Solution Overview } & 6\end{array}$

$\begin{array}{lll}3 & \text { Ethics of Twitter Observation } & 8\end{array}$

3.1 Twitter Terms of Service . . . . . . . . . . . . . . . . . . . . . . . 8

$\begin{array}{lll}4 \text { SPOONS Requirements } & 10\end{array}$

5 Contributions and Organization 12

6 Background \& Related Work 14

6.1 Twitter Traffic Analysis . . . . . . . . . . . . . . . . . . . . 14

6.1 .1 Twitter Classification . . . . . . . . . . . . . . . . . 15

6.1 .2 Twitter Anomaly Detection . . . . . . . . . . . . . 15

6.2 Classifiers $\ldots \ldots \ldots \ldots \ldots \ldots$

$6.2 .1 \quad$ Naive Baves . . . . . . . . . . . . . . . . . . 17

$6.2 .2 \quad$ Bayes Net . . . . . . . . . . . . . . . . . . . . . . 18

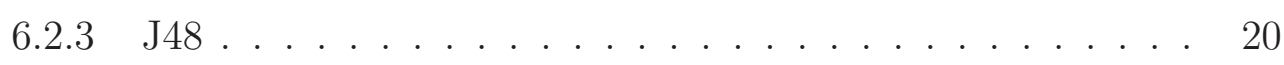

$6.2 .4 \quad$ K-Nearest Neighbors . . . . . . . . . . . . . . . . . . 21

6.2 .5 Support Vector Machines . . . . . . . . . . . . . . . . . 22

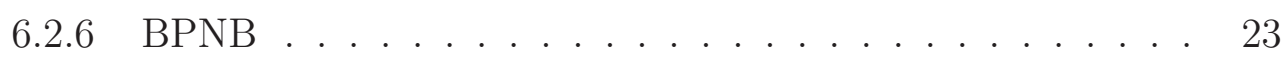


$6.2 .7 \quad$ WEKA . . . . . . . . . . . . . . . . . . . 24

6.3 Singletons . . . . . . . . . . . . . . . . . . . . . . 24

6.4 Accuracy Measures . . . . . . . . . . . . . . . . . . . 25

6.4 .1 Recall . . . . . . . . . . . . . . . . . . . 26

6.4 .2 Precision . . . . . . . . . . . . . . . . . . . . . . . 26

$6.4 .3 \quad$ F Score . . . . . . . . . . . . . . . . . . . 27

6.4 .4 Coverage . . . . . . . . . . . . . . . . . . . . . . . 27

$\begin{array}{lll}7 & \text { Twitter API } & 29\end{array}$

$7.1 \quad$ Rate Limiting . . . . . . . . . . . . . . . . . . . . . . . . . . 29

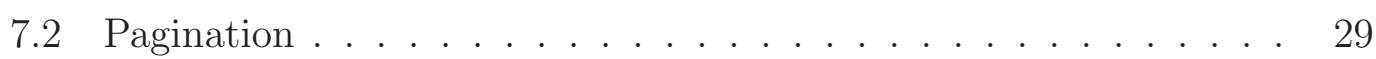

7.3 Query Anatomy . . . . . . . . . . . . . . . . . . . . 30

7.4 Result Anatomy . . . . . . . . . . . . . . . . . . . . . . . . 31

2 SPOONS Architecture 33

8 Architecture Breakdown 34

9 Framework Architecture 36

9.1 High Level Solution . . . . . . . . . . . . . . . . . . . . . . 36

9.1 .1 Framework Overview . . . . . . . . . . . . . . . . . 37

9.2 Gatherers . . . . . . . . . . . . . . . . . . . . . . 38

9.2 .1 Twitter Holes . . . . . . . . . . . . . . . . . . . . . . . . 38

9.3 Processors . . . . . . . . . . . . . . . . . . . . . . . . . . . . . . 39

$9.4 \quad$ Analysis Pipelines . . . . . . . . . . . . . . . . . . . . . . 40

9.5 Tasks . . . . . . . . . . . . . . . . . . . 41

9.6 Modelers . . . . . . . . . . . . . . . . . . . . . . . . . . . . . . . . . . 41

9.6 .1 Predictors . . . . . . . . . . . . . . . . . . 42

9.6 .2 Counters . . . . . . . . . . . . . . . . . 42

9.7 Monitors . . . . . . . . . . . . . . . . . . . . . . . . 42

9.7 .1 Auto-Tuning . . . . . . . . . . . . . . . . . 42

9.7 .2 Resistance . . . . . . . . . . . . . . . . . . . . . . 43

9.7 .3 Smoothers . . . . . . . . . . . . . . . . . . . . 45 


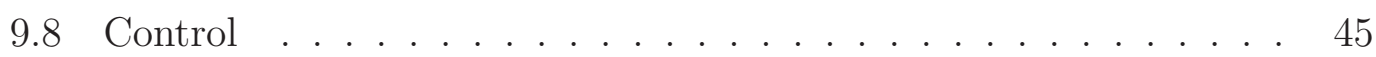

9.8 .1 Master Control . . . . . . . . . . . . . . . . 46

$9.8 .2 \quad$ Worker Control . . . . . . . . . . . . . . . . . . 47

$9.8 .3 \quad$ Single Control . . . . . . . . . . . . . . . . . . . . . 47

10 Distributed Computation Model 48

10.1 Distribution Requirements . . . . . . . . . . . . . . . . . . . . 49

10.2 Distribution Assumptions . . . . . . . . . . . . . . 51

10.2 .1 Same Data . . . . . . . . . . . . . . . . . . . . 51

10.2.2 Uniquely Referenced Tasks . . . . . . . . . . . . . . . 52

10.3 Distributable Tasks . . . . . . . . . . . . . . . . . . . 52

10.3 .1 Master Contro . . . . . . . . . . . . . . . . . . 52

10.3 .2 Worker Contro . . . . . . . . . . . . . . . . . . 53

10.3 .3 Single Control . . . . . . . . . . . . . . . . . . . . 53

10.4 Shared Properties . . . . . . . . . . . . . . . . . . . . 53

11 Database 55

11.1 Tables and Schemas . . . . . . . . . . . . . . . 56

11.1 .1 Data Flow . . . . . . . . . . . . . . . . 57

11.1 .2 Tweets Table . . . . . . . . . . . . . . . . . . 57

11.2 UI Stored Procedures . . . . . . . . . . . . . . . . . . . . . 58

11.2 .1 Expected Schemas . . . . . . . . . . . . . 60

3 Analysis 162

12 Classifiers 63

12.1 Why Classification? . . . . . . . . . . . . . . . . 63

12.2 Classification Roadmap . . . . . . . . . . . . . . . . . 66

12.3 Tweet Classes . . . . . . . . . . . . . . . . . . . . 66

12.3 .1 Tweet Groups . . . . . . . . . . . . . . . . . . . . . 67

12.4 WEKA Classifiers . . . . . . . . . . . . . . . . . . . . 69

12.5 Non-WEKA Classifiers . . . . . . . . . . . . . . 70

12.6 Text Pre-Processing . . . . . . . . . . . . . . . . . . . . . . . . 70 
12.6 .1 Text Filtering . . . . . . . . . . . . . . . . . . 71

12.7 Training Set . . . . . . . . . . . . . . . . . . . . 75

12.8 Evaluation . . . . . . . . . . . . . . . . . . . . . . . 75

12.8.1 Confusion Matrices . . . . . . . . . . . . . 77

12.8 .2 Results . . . . . . . . . . . . . . . . . . . . . 78

12.8 .3 Compressed Results . . . . . . . . . . . . . . . . . 79

13 Outage Detection 83

13.1 Ground Truth . . . . . . . . . . . . . . . . . . . . . . . 83

13.2 Success Metrics $\ldots \ldots \ldots$. . . . . . . . . . . . . . 83

13.2 .1 Adjusted Score . . . . . . . . . . . . . . . . . . . . 84

13.3 Monitors . . . . . . . . . . . . . . . . . . . . . . 85

13.3 .1 Baseline Monitor . . . . . . . . . . . . . . . . . 85

13.3.2 Windowed Standard Deviation Monitor . . . . . . . . . 86

13.3.3 Weekly Offset Windowed Standard Deviation Monitor . . 88

13.3.4 Mean Squared Error Monitor ．. . . . . . . . . . . . . 89

13.3 .5 Ratio Monitor . . . . . . . . . . . . . . . . . . . 90

13.3 .6 Class Correlation Monitor . . . . . . . . . . . . . . . . 91

13.4 Evaluation . . . . . . . . . . . . . . . . . . . . . 93

13.5 Results . . . . . . . . . . . . . . . . . . . . . 94

4 Conclusions 98

\begin{tabular}{ll}
\hline 14 Conclusions & 99
\end{tabular}

14.1 The SPOONS System . . . . . . . . . . . . . . . . . . . . . 99

14.2 Tweet Classification . . . . . . . . . . . . . . . . 100

14.3 Outage Detection . . . . . . . . . . . . . . . . . 100

14.4 Fulfilled Requirements . . . . . . . . . . . . . . . . 100

15 Current Limitations of SPOONS 102

16 Current and Future Work 104

16.1 WEKA Classifier Reimplementation . . . . . . . . . . . . . . . . . 104

16.2 Advanced Sentiment Analysis . . . . . . . . . . . . . . . . . 104 
16.3 SPOONS Scaling . . . . . . . . . . . . . . . . . 105

\begin{tabular}{ll}
\hline Bibliography & 106
\end{tabular}

A SPOONS Database Schema Highlights 111

A.1 DATA tweets . . . . . . . . . . . . . . . . . . . . . . . . 111

\begin{tabular}{ll}
\hline B Full Classifier Evaluation Results & 116
\end{tabular}

\begin{tabular}{ll}
\hline C Full Outage Detection Evaluation Results & 125
\end{tabular}

D Full Training Set 152 


\section{List of Tables}

9.1 Fighting Resistance Parameters . . . . . . . . . . . . . . . 43

9.2 Continuous Resistance Parameters . . . . . . . . . . . . . . . . . 44

9.3 Window Resistance Parameters . . . . . . . . . . . . . . . . . 44

9.4 Moving Mean Smoother Parameters . . . . . . . . . . . . . . . . . 45

11.1 Database Tweet Attributes - The database attributes used to describe tweets. . . . . . . . . . . . . . . . 59

11.2 Stored Procedure UI Expected Schema - The different types of schemas that the UI looks for in RESULT tables. . . . . . . . . . 60

12.1 Tweet Class Examples - Examples of the types of tweets that go with each class. . . . . . . . . . . . . . . . . . . . . . 68

12.2 Netflix-related Twitter Traffic - Overview of the Netflix-related Twitter post training set used to train classifiers in SPOONS. . . 76

12.3 Classifier Evaluation Combinations - The cross product of the classifier and filtering will be used to evaluate the classifiers. . . . 76

12.4 Example Classification Confusion Matrix - An example confusion matrix for a classifier. The undect ded class was removed because there were no tweets in that class. . . . . . . . . . . . . 78

12.5 Uncompressed Misclassification Example - The SMO results with no filtering. Every misclassification that would map to the other group is bold. . . . . . . . . . . . . . . . . 79

12.6 Misclassified Snafu - A sample compressed classification confusion matrix showing misclassified snafu tweets. . . . . . . . . . . 80

12.7 Uncompressed Classification Results Summary . . . . . . . . . . . 81

12.8 Compressed Classification Results Summary . . . . . . . . . . . . 82 
13.1 Baseline Monitor Parameters . . . . . . . . . . . . . . . . . . . 85

13.2 Baseline Monitor Input Arguments ～. . . . . . . . . . . . 85

13.3 Windowed Standard Deviation Monitor Parameters . . . . . . . . 86

13.4 Windowed Standard Deviation Monitor Input Arguments . . . . . 86

13.5 Windowed Standard Deviation Monitor Example - An example of how the Windowed Standard Deviation Monitor determines when to alert. . . . . . . . . . . . . . . . . . . 87

13.6 Weekly Offset Windowed Standard Deviation Monitor Parameters 88

13.7 Weekly Offset Windowed Standard Deviation Monitor Input Arguments . . . . . . . . . . . . . . . . 88

13.8 Mean Squared Error Monitor Parameters . . . . . . . . . . . . . 89

13.9 Mean Squared Error Monitor Input Arguments . . . . . . . . . . 89

13.10MSE Monitor Example - An example of how the MSE Monitor determines when to alert. . . . . . . . . . . . . . 90

13.11Ratio Monitor Parameters . . . . . . . . . . . . . . . . 90

13.12Ratio Monitor Input Arguments . . . . . . . . . . . . . . . . 91

13.13Class Correlation Monitor Parameters . . . . . . . . . . . . . . . . 91

13.14Class Correlation Monitor Input Arguments . . . . . . . . . . . 92

13.15Outage Detection Evaluation Combinations - The cross product of the classifier and Monitor will be used to evaluate the outage detection methods. . . . . . . . . . . . . . . . . . . . . 93

13.16Example Outage Detection Results - An example of the output for an outage detection method. . . . . . . . . . . . . . . . 94

13.17Outage Detection Results Summary . . . . . . . . . . . . . . . 97

B.1 Uncompressed, None Filter Classification Confusion Matrices . . . 117

B.0 Uncompressed, None Filter Classification Confusion Matrices Cont. 118

B.-1 Uncompressed, None Filter Classification Confusion Matrices Cont. 119

B.0 Uncompressed, Full Filter Classification Confusion Matrices . . . 120

B.-1 Uncompressed, Full Filter Classification Confusion Matrices Cont. 121

B.-2 Uncompressed, Full Filter Classification Confusion Matrices Cont. 122

B.-1 Compressed, None Filter Classification Confusion Matrices . . . . 123

B.0 Compressed. Full Filter Classification Confusion Matrices . . . . . 124 
C.1 WeeklyWindowStdDev SMO (Full Filtering) Results . . . . . . . 125

C.2 WeeklyWindowStdDev BinaryNaiveBayes (Full Filtering) Results 126

C.3 WeeklyWindowStdDev J48 (Full Filtering) Results . . . . . . . . 126

C.4 WeeklyWindowStdDev BPNB (No Filtering) Results . . . . . . 127

C.5 WeeklyWindowStdDev BinarySMO (No Filtering) Results . . . . 127

C.6 WeeklyWindowStdDev SMO (No Filtering) Results . . . . . . . . 128

C.7 WeeklyWindowStdDev BinaryNaiveBaves (No Filtering) Results . 128

C.8 WeeklyWindowStdDev NaiveBaves (No Filtering) Results . . . . . 129

C.9 WeeklyWindowStdDev BinarySMO (Full Filtering) Results . . . . 129

C.10 WeeklyWindowStdDev BPNB (Full Filtering) Results . . . . . . . 130

C.11 Baseline BinarySMO (Full Filtering) Results . . . . . . . . . . . 130

C.12 Ratio J48 (Full Filtering) Results . . . . . . . . . . . . . . . . . 130

C.13 MSE BinaryNaiveBayes (No Filtering) Results . . . . . . . . . . 131

C.14 MSE NaiveBayes (No Filtering) Results . . . . . . . . . . . . . . 131

C.15 Baseline J48 (Full Filtering) Results . . . . . . . . . . . . . . 132

C.16 Baseline SMO (No Filtering) Results . . . . . . . . . . . . . . 132

C.17 Baseline BinaryNaiveBaves (Full Filtering) Results . . . . . . . 132

C.18 Baseline NaiveBaves (No Filtering) Results . . . . . . . . . . . . 133

C.19 Baseline BinaryNaiveBaves (No Filtering) Results . . . . . . . . . 133

C.20 MSE BinarySMO (No Filtering) Results . . . . . . . . . . . . . 133

C.21 MSE BinarySMO (Full Filtering) Results . . . . . . . . . . . . . 134

C.22 Baseline BinarySMO (No Filtering) Results . . . . . . . . . 134

C.23 MSE SMO (No Filtering) Results . . . . . . . . . . . . . . 135

C.24 Baseline BPNB (Full Filtering) Results . . . . . . . . . . . . . . 135

C.25 Ratio BPNB (Full Filtering) Results . . . . . . . . . . . . 136

C.26 Baseline SMO (Full Filtering) Results . . . . . . . . . . . . . 136

C.27 Ratio SMO (Full Filtering) Results . . . . . . . . . . . . 136

C.28 Baseline BPNB (No Filtering) Results . . . . . . . . . . . 137

C.29 Ratio SMO (No Filtering) Results . . . . . . . . . . . . . . . 137

C.30 Ratio BinaryNaiveBaves (No Filtering) Results . . . . . . . . 137

C.31 Ratio NaiveBaves (No Filtering) Results . . . . . . . . . . 138 
C.32 Ratio BinarySMO (Full Filtering) Results . . . . . . . . . . . 138

C.33 Correlation SMO (Full Filtering) Results . . . . . . . . . . . 138

C.34 Ratio BinarySMO (No Filtering) Results . . . . . . . . . . . . . 139

C.35 Ratio BPNB (No Filtering) Results . . . . . . . . . . . . . . . 139

C.36 Correlation BPNB (Full Filtering) Results . . . . . . . . . . . 139

C.37 Ratio BinaryNaiveBayes (Full Filtering) Results . . . . . . . . . . 140

C.38 Correlation BPNB (No Filtering) Results . . . . . . . . . . . . . 140

C.39 Correlation BinarvNaiveBaves (Full Filtering) Results . . . . . . . 141

C.40 Correlation J48 (Full Filtering) Results . . . . . . . . . . . . . 141

C.41 Correlation SMO (No Filtering) Results . . . . . . . . . . . . 142

C.42 MSE J48 (Full Filtering) Results . . . . . . . . . . . . . . . 142

C.43 MSE BPNB (No Filtering) Results . . . . . . . . . . . . . . . 143

C.44 Correlation BinaryNaiveBayes (No Filtering) Results . . . . . . . 143

C.45 Correlation BinarySMO (Full Filtering) Results . . . . . . . . . . 144

C.46 Correlation BinarySMO (No Filtering) Results . . . . . . . . . . . 144

C.47 Correlation NaiveBaves (No Filtering) Results . . . . . . . . . . 145

C.48 MSE SMO (Full Filtering) Results . . . . . . . . . . . . . 145

C.49 MSE BinarvNaiveBaves (Full Filtering) Results . . . . . . . . . 146

C.50 MSE BPNB (Full Filtering) Results . . . . . . . . . . . . . . . 146

C.51 WindowStdDev BinarvSMO (No Filtering) Results . . . . . . . . 147

C.52 WindowStdDev NaiveBaves (No Filtering) Results . . . . . . . . . 147

C.53 WindowStdDev BinaryNaiveBayes (No Filtering) Results . . . . . 148

C.54 WindowStdDev BinarySMO (Full Filtering) Results . . . . . . . . 148

C.55 WindowStdDev SMO (No Filtering) Results . . . . . . . . . . 149

C.56 WindowStdDev J48 (Full Filtering) Results . . . . . . . . . . 149

C.57 WindowStdDev BPNB (No Filtering) Results . . . . . . . . . 150

C.58 WindowStdDev BPNB (Full Filtering) Results . . . . . . . . . . . 150

C.59 WindowStdDev BinaryNaiveBaves (Full Filtering) Results . . . . 151

C.60 WindowStdDev SMO (Full Filtering) Results. . . . . . . . . . . 151 


\section{List of Figures}

1.1 Outage Tweets Example - Tweets posted on March 9. 2011 during a disruption of Netflix streaming to the Nintendo Wii console.

2.1 Svstem Concept Diagram - This svstem concept diagram shows the general flow of processing done in the SPOONS system. . . .

6.1 Simple Bayes Net - A simple Bayesian Network modeling the chance of going on a picnic giyen the season and weather. The season affects the weather and both the season and weather affect the chance of going on a picnic. . . . . . . . . . . . . . . . .

6.2 Baves Net With Probabilities - The simple Bavesian Network augmented with the probability functions for each node. . . . . . 19

6.3 Simple Decision Tree - A simple decision tree trying to answer the question of whether or not to go on a picnic. . . . . . . . . . 20

6.4 K-Nearest Neighbors - A simple example of KNN. If $k=3$, then the query point (the star) will be classified as a triangle. However, if $k=5$ then the query point will be classified as a square. . . . .

6.5 Support Vector Machine - A simple example of a support vector machine. The SVM chose a partition that maximizes the margin between the squares and triangles. . . . . . . . . . . . . . . .

6.6 Base Relative Singleton - An example base relative singleton inheritance hierarchy. Note that instantiating any child removes the ability to instantiate any other part of the hierarchy. . . . . . . . 25

6.7 Child Relative Singleton - An example child relative singleton inheritance hierarchy. Note that any child can be instantiated, but only once. . . . . . . . . . . . . . . . . . 25

6.8 Coverage Example - A long alert producing an unjustly high precision and recall. . . . . . . . . . . . . . . . 28 
7.1 Twitter API Query - The structure of a typical query to the Twitter API. . . . . . . . . . . . . . . . 30

7.2 Twitter Search API Result - A JSON result from the Twitter Search API . . . . . . . . . . . . . . . . . 32

8.1 SPOONS Framework Architecture - The flow of control and data through the SPOONS framework system. . . . . . . . . . . . 35

8.2 SPOONS UI - The web UI for SPOONS. . . . . . . . . . . 35

9.1 Twitter Holes - One server in a hole is covered by two other gathering servers. . . . . . . . . . . . . . . . 39

10.1 SPOONS Server Architecture - The server architecture of the SPOONS system. . . . . . . . . . . . . . . . . 49

10.2 SPOONS Distributable Task Flow - The control flow for distributable tasks. . . . . . . . . . . . . . . . 50

11.1 Database Data Flow - The flow of data through the different types of tables in SPOONS. . . . . . . . . . . . 58

12.1 Normal Traffic - A week's worth of Netflix-related Twitter traffic. Notice the daily periodicity. . . . . . . . . . . . . . . . 64

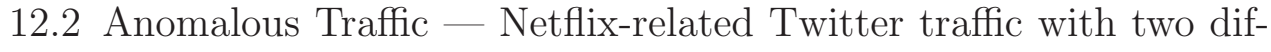
ferent anomalies. . . . . . . . . . . . . . . . . 64

12.3 Linkless Anomalous Traffic - The same traffic shown in Figure 12.2. with an additional line showing Netflix-related Twitter traffic that does not contain a URL. . . . . . . . . . . . . . . . 65

12.4 Classified Traffic — The same traffic shown in Figure 12.2, with two addition lines: the volume of tweets lassified as "Bad" and the volume of tweets classified as "Media". . . . . . . . . . . . . 65

12.5 SPOONS Groups - The different volumes for different tweet classes during an outage (left) and media event (right). . . . . . . . .

12.6 Title Trie Walk - A sample trie of Frankenstein movie titles. The solid lines show what nodes the search for "Frankenstein Meets The Wolf Man" would traverse. . . . . . . . . . . . . . . 73 


\section{Part 1}

\section{Introduction}




\section{Chapter 1}

\section{Problem: Swift Perception Of Online Negative Situations}

Twitter is an immensely popular micro-blogging service. According to the Twitter, as of March $14^{\text {th }}$ 2011, approximately one billion micro-posts, tweets, were being posted per week [28]. Because of the low time and effort cost of tweeting, only a few seconds from a smart phone, Twitter users post tweets about almost every aspect of their daily lives. Because of this large stream of information, Twitter makes an excellent source of information for data miners interested in real-time events. Already, researchers have been using Twitter to attempt to track and model disease outbreaks [5], earthquakes [16], and the stock market [11].

Netflix is the one of the largest online Internet subscription service for streaming movies and television shows. Netflix has over 25 million subscribers watching media streamed to over 450 different platforms. Even a short disruption of their streaming service can affect millions of users. Therefore, quickly detecting service 
outages is essential to keep customers happy. However, service outage detection is no trivial matter in Netflix's environment. In addition to constantly streaming thousands of different videos to hundreds of different platforms, Netflix also has to deal with problems caused by most of their infrastructure being hosted in the cloud with Amazon Web Services (AWS).

Netflix saw the power in Twitter as a potential data source for detecting service outages that is orthogonal to their current, more traditional outage detection methods. Currently, Netflix utilizes four different methods for detecting outages:

Internal Monitoring Systems. Like any sizable service providing company, Netflix utilizes many different internal monitoring systems to detect service outages. However, there are some classes of problems that are difficult to solve with internal monitoring. These problems include corrupt video files or a problem on a third-party delivery platform such as Roku or AppleTV. These problems are obvious to the end user, but very difficult to detect internally. In addition, the internal monitoring systems share the same infrastructure as the service providing system. Therefore, a problem in the infrastructure can cause both systems to go down at the same time.

External Monitoring Systems. Netflix contracts with external services that can periodically probe its systems to try and detect problems. However, this model too has problems. There are many problems that cannot be seen from an external probe. Also, if this system probes too often then it is taking compute time away from the servers that are trying to deliver content to end users. 
Customer Service. Calls to customer service are a very straight-forward way to detect outages. Unfortunately, this method is very slow and inconsistent. It takes a lot of frustration to get a user to lookup a phone number and complain.

Manual Twitter Observation. Manual observation shows that there is usually a response on Twitter when Netflix suffers a service outage. Figure 1.1]shows some tweets that occurred during a disruption of Netflix's service to the Nintendo Wii. However without any infrastructure, Twitter observation is slow and inconsistent. It is also very time consuming to have someone constantly watching Twitter for signs of an outage.

Given all these deficiencies Netflix wanted a monitoring system that is separate from their infrastructure, fast, and does not require any human intervention [17]. 


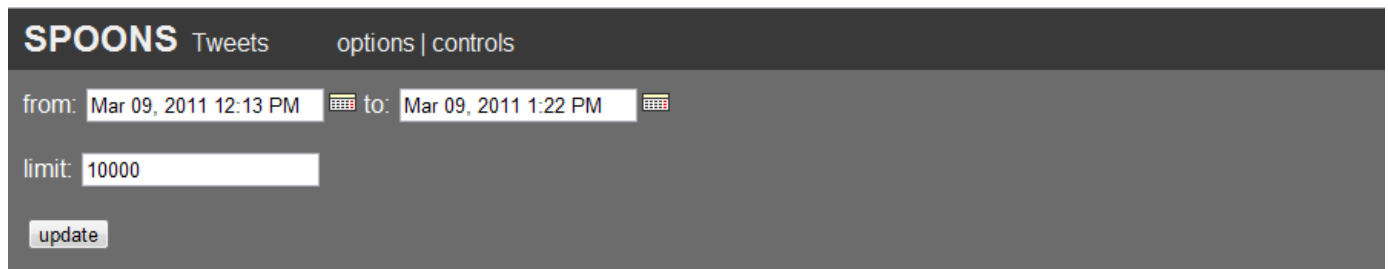

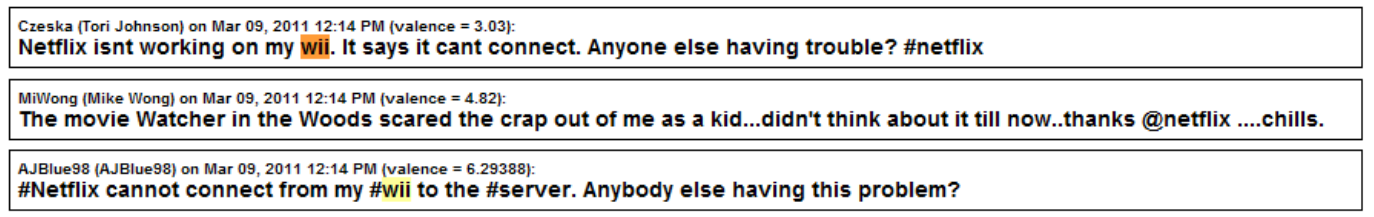

Daily_Pinch (Lisa Frame) on Mar 09, 2011 01:19 PM (valence = 6.29377):
@Netflixhelps My netflix streaming is down. I've rebooted by wii, turned off entire system, my internet is working fine on wii.

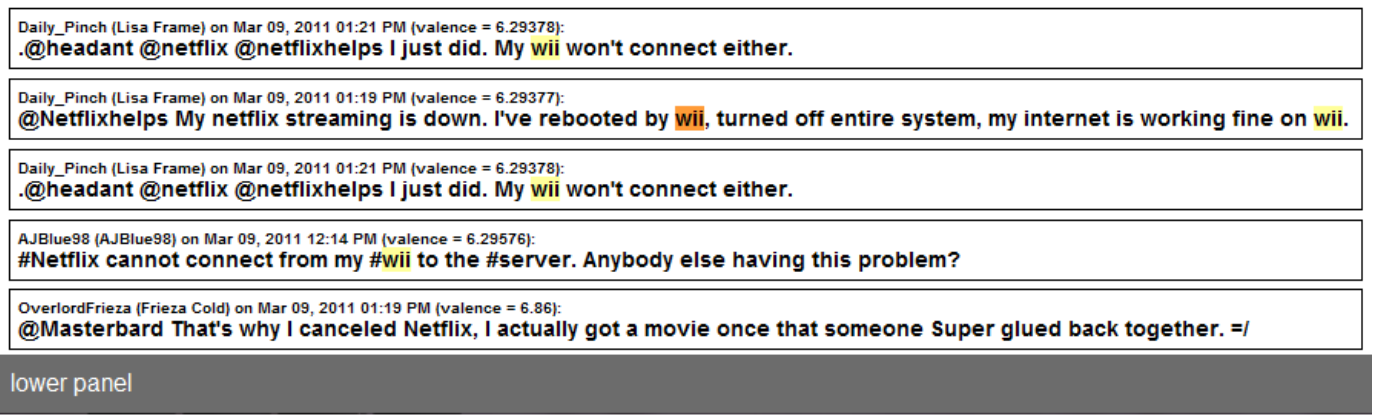

Figure 1.1: Outage Tweets Example — Tweets posted on March 9, 2011 during a disruption of Netflix streaming to the Nintendo Wii console. 


\section{Chapter 2}

\section{Solution Overview}

SPOONS (Swift Perception Of Online Negative Situations) is a system that is designed to use tweets to detect outages in Netflix content delivery systems. At present, the system supports a wide variety of detection methods that use some combination of time series analysis, classification, natural language processing, sentiment analysis, and filtering.

Figure 2.1 shows how the SPOONS system can be divided into three main parts: input; analysis pipelines; and output. The inputs are tweets gathered from Twitter. Then the analysis pipelines use a combination of sentiment estimation, classification, and traffic volume analysis to detect when an outage is occurring. The outputs of the system are: email alerts to Netflix engineers, and a web UI that displays information about the outage. 


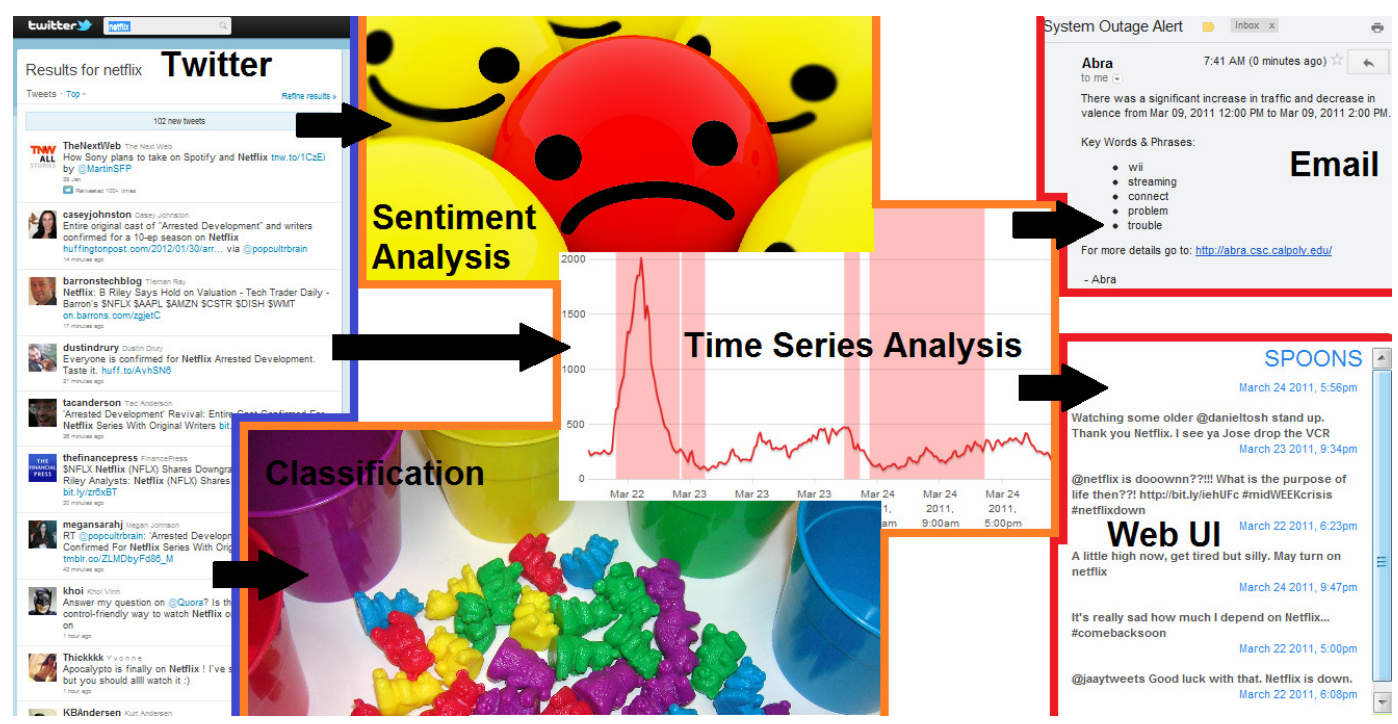

Figure 2.1: System Concept Diagram - This system concept diagram shows the general flow of processing done in the SPOONS system. 


\section{Chapter 3}

\section{Ethics of Twitter Observation}

The work in this project uses content that users post on Twitter without their knowledge. This monitoring system isn't being announced to the public because widespread knowledge of it would increase the likelihood of a malicious attack. This practice may lead to concerns about the level of privacy or ownership being provided to Twitter users regarding the content they post through the Twitter services. The goal of this section is to address these concerns by providing more information about the Twitter services and how the SPOONS system and this work uses the tweets.

\subsection{Twitter Terms of Service}

According to Twitter Terms of Service [29] agreement that everyone accepts automatically by accessing or using Twitter services:

"You retain your rights to any Content you submit, post or display on or through the Services. By submitting, posting or displaying Content on or through 
the Services, you grant us a worldwide, non-exclusive, royalty-free license (with the right to sublicense) to use, copy, reproduce, process, adapt, modify, publish, transmit, display and distribute such Content in any and all media or distribution methods (now known or later developed)."

"This license is you authorizing us to make your Tweets available to the rest of the world and to let others do the same."

"You agree that this license includes the right for Twitter to make such Content available to other companies, organizations or individuals who partner with Twitter for the syndication, broadcast, distribution or publication of such Content on other media and services, subject to our terms and conditions for such Content use."

"We encourage and permit broad reuse of Content. The Twitter API exists to enable this."

"Such additional uses by Twitter, or other companies, organizations or individuals who partner with Twitter, may be made with no compensation paid to you with respect to the Content that you submit, post, transmit or otherwise make available through the Services."

In short, Twitter takes ownership of user tweets as soon as they are posted on Twitter. Using the Twitter API allows SPOONS to obtain the tweets with the consent of Twitter. Therefore, the collection and analysis of Twitter data by SPOONS is well within the Twitter Terms of Service. 


\section{Chapter 4}

\section{SPOONS Requirements}

Netflix has provided the following set of key requirements to be met by the SPOONS system:

Structural Independence. The outage detection system shall be structurally independent of both the software and the hardware infrastructure used by Netflix. It shall rely only on information that is publicly available and free for use. This ensures that the outage detection system stays up even when any or all Netflix servers are experiencing downtime.

Use of Amazon Web Services. Netflix is one of the largest customers of Amazon.com's cloud computing service, Amazon Web Services (AWS). AWS allows users to create new cloud machines (instances) in many regions throughout the world. The outage detection system shall be deployed on one or more AWS servers that are operationally independent of other AWS servers used by Netflix. Using a cloud solution allows the outage detection and alert system to be deployable on a global scale. 
Real-Time. Netflix's streaming services run in real-time and any downtime has an immediate impact on customers. To minimize that impact, the outage detection system shall notify Netflix of detected outages as soon as possible.

Precise Outage Detection. The number of non-outage situations that raise an alert shall be minimized. While a small number of false positives detected in real-time may be acceptable, the outage detection system shall detect outages and generate alerts with as high precision as possible.

Comprehensive Outage Detection. Not all Netflix service outages generate a signal on Twitter. Those that don't may be allowed to go unnoticed by the outage detection system (as the system has no basis for detecting them), but any outage that causes a signal on Twitter shall be detected.

User-Friendly Online UI. The outage detection and alert system shall have an easy-to-use, informative, online UI which shall provide Netflix employees with real-time information and historic data about the state of Netflix according to Twitter. The information provided shall include:

- times of outages;

- times of other anomalous events;

- current and recent Netflix-related Twitter traffic trends;

- and samples of Netflix-related tweets. 


\section{Chapter 5}

\section{Contributions and Organization}

SPOONS is a continual team effort and has been touched and improved by many different people. The idea originated at Netflix and was passed to the ABRA team at Cal Poly. The ABRA team has published a paper on SPOONS 2. In addition, Cailin Cushing defended a thesis on a part of SPOONS devoted to outage detection through sentiment analysis [6].

The main contributions of this work are as follows:

- Design and implementation of the SPOONS system.

- Design and implementation of the SPOONS framework.

- Design and implementation of the SPOONS server architecture.

- Design and implementation of the SPOONS distributed computation model.

- Design of the SPOONS database structure and all table schemas.

- Design, implementation, and evaluation of all SPOONS classification based outage detection methods. 
The rest of the paper is organized as follows. Chapter [ 6 covers background and related work. Part 2 discusses the architecture of SPOONS. Part 3 discuss the work done by the SPOONS system to detect outages. With Chapter 12 focusing on the details of the classifiers used in SPOONS, and Chapter 13 extending the problem of classification to full outage detection. Part 4 wraps up the paper. 


\section{Chapter 6}

\section{Background \& Related Work}

\subsection{Twitter Traffic Analysis}

Twitter proves to be a great resource for data mining because of the large number of real-time, posts from millions of users. However, tweets can be very difficult to work with because they suffer from three large drawbacks:

Length. Tweets can only be 140 characters long. This limit severely restricts the possible information content of a tweet. Compared to more traditional media sources, e.g. news articles, the text of tweets contain almost no information. Although this makes it very difficult to do naive text classification on tweets, Twitter users have found ways to increase their information density. Links to news stories, slang, and Twitter symbols (see Section 12.6.1) help Twitter users express more with fewer characters.

Informal Language. Informal language, e.g. slang, jargon, and abbreviations, is common place on Twitter. The use of informal language can be partially 
attributed to the strict character limit. Informal language can be difficult to deal with because it less likely to appear in well established Natural Language Processing corpora. Not having a corpus forces researchers to either only use unsupervised methods, or build their own corpus.

Typos. The nature of Twitter is very informal for most users whose tweets are only read by their friends. This informal environment and the large amount of tweets coming from hand-held devices without a traditional keyboard leads to many tweets containing typos. Typos make text analysis difficult because they obfuscate words and increases the number of unique words.

\subsubsection{Twitter Classification}

There has been much work in using classifiers on both tweets and Twitter users. Most of the classification efforts has gone into trying to determine the sentiment, the general feeling, of a tweet [12] 18] 27] [31. Raz et al. tackle the task of classifying humorous tweets as a specific type of humor such as irony, observational, or wordplay [26]. The traditional text classification task of topic modeling has also been attempted various times [10] 33. Instead of trying to classify tweets, Pennacchiotti et al. try to classify user associations from their tweets [21].

\subsubsection{Twitter Anomaly Detection}

Levchenko et al.[15] created a system that uses tweets to detect outages in several widely used Web services such as Amazon, Gmail, Google, PayPal, Netflix, Youtube, Facebook, Wikipedia, and Flickr. They describe Twitter users as acting 
as millions of sensors who have a large breadth and flexibility of in the definition of failure. The detection mechanism employed in this work is fairly straightforward. A collection of tweets that either contain the phrase " $\mathrm{X}$ is down" or a "\#Xfail" hashtag, where "X" is the name of a service (e.g., "\#netflixfail") is gathered. The traffic is compared against expected traffic to determine if there is an outage.

Levchenko et al. were only able to validate a subset of their detected events because a full validation would require a list of all outages during 2009 for every service that they were monitoring. So while the events they were able to verify indicate that the system can detect outages, the full effectiveness of their method is still largely unknown.

\subsection{Classifiers}

SPOONS uses a variety of different classifiers for text classification. This section gives an overview of each different type of classifier used.

\section{Formal Definition}

The classification problem that the classifiers are trying to solve can be defined as follows:

Given a set of documents $D$

$$
D=\{d \mid d \in D\}
$$

where each document $d$ is a vector of $n$ features

$$
d=\left(\mathrm{f}_{1}, \mathrm{f}_{2}, \ldots, \mathrm{f}_{n}\right)
$$


and a set of classes $C$ where

$$
C=\{c \mid c \in C\}
$$

We want to associate each document with a class based on the patterns observed in a training set $T$ of already classified documents.

$$
T=\{(d, c) \mid c \in C\}
$$

\subsubsection{Naive Bayes}

Naive Bayes classifier works by applying the Bayes' theorem with the assumption that the probability of each feature in a document is independent from the probability of any other feature appearing in the same document. [13] [8]

The Bayes' theorem states that the probability of observing class $c$ given document $d, \operatorname{Pr}(c \mid d)$, can be represented as:

$$
\operatorname{Pr}(c \mid d)=\frac{\operatorname{Pr}(c) \cdot \operatorname{Pr}(d \mid c)}{\operatorname{Pr}(d)}
$$

$\operatorname{Pr}(c)$ is the prior probability of class $c$, that is, the probability of observing $c$ regardless of the document attached to it. When training the classifier, this is just the percentage of times that the class appeared in the training set.

$\operatorname{Pr}(d)$ is the prior probability of document $d$. Like $\operatorname{Pr}(c)$, it is just the probability of observing the collection of features $d$ regardless of the class associated with it. Note that for classification, it is not necessary to compute $\operatorname{Pr}(d)$ because it is constant among all documents and classes. A classifier can just choose the class with the largest $\operatorname{Pr}(c) \cdot \operatorname{Pr}(d \mid c)$ term.

$\operatorname{Pr}(d \mid c)$ is the probability of observing document $d$ given that $d$ is already recognized as belonging to class $c$. Remember that document $d$ is really just a 
vector of $n$ features, $\left(\mathrm{f}_{1}, \mathrm{f}_{2}, \ldots, \mathrm{f}_{n}\right)$. Assuming conditional independence (the naive part in Naive Bayes), $\operatorname{Pr}(d \mid c)$ can be constructed as a product of the probability of observing each feature in $d$ :

$$
\operatorname{Pr}(d \mid c)=\operatorname{Pr}\left(\mathrm{f}_{1} \mid c\right) \cdot \operatorname{Pr}\left(\mathrm{f}_{2} \mid c\right) \cdot \ldots \cdot \operatorname{Pr}\left(\mathrm{f}_{n} \mid c\right)=\prod_{i=1}^{n} \operatorname{Pr}\left(\mathrm{f}_{i} \mid c\right)
$$

Now the last step is to estimate the conditional probabilities of the $n$ features. When dealing with discrete features, then estimating $\operatorname{Pr}\left(\mathrm{f}_{m} \mid c\right)(1 \leq m \leq n)$ can be done by finding the percentage of training documents that contain feature $\mathrm{f}_{m}$ and have class $c$.

\subsubsection{Bayes Net}

A Bayesian Network is a probabilistic, directed acyclic graphs that represents a set of random variables and their conditional probabilities. In a Bayesian Network, the collection of incoming edges represent the conditional probability distribution between two random variables. Each node represents a variable and a probability function that takes as input the state of the node's parents. 20] [19]

Figure 6.1 shows a simple Bayesian Network that models the chance of going on a picnic. Note that whether or not it is Spring affects the chance of it raining; and both the season and weather affect the chance of going on a picnic.

Figure 6.2 shows the probability distributions for the network. The chance of the season being Spring is fully independent, and therefore takes no parameters into its probability function. However, the weather and picnic decision takes one and two input parameters respectively. 


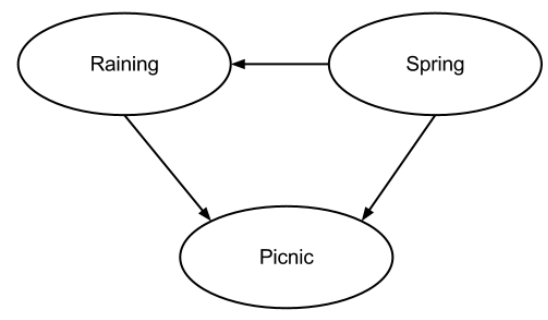

Figure 6.1: Simple Bayes Net — A simple Bayesian Network modeling the chance of going on a picnic given the season and weather. The season affects the weather and both the season and weather affect the chance of going on a picnic.

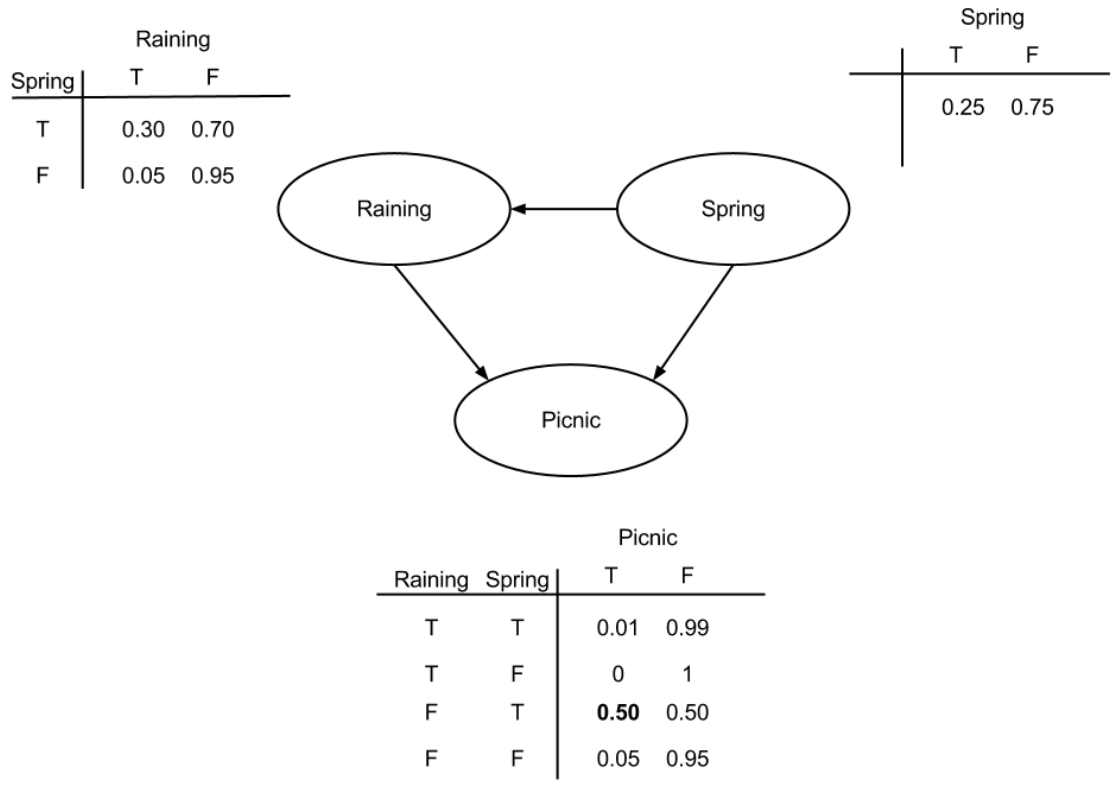

Figure 6.2: Bayes Net With Probabilities — The simple Bayesian Network augmented with the probability functions for each node. 


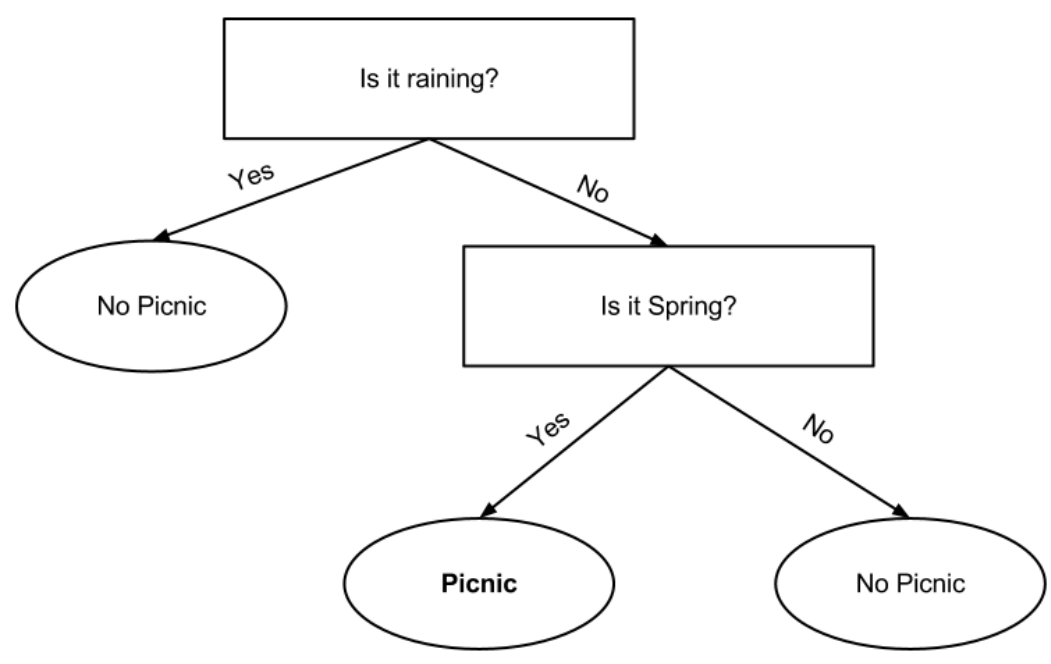

Figure 6.3: Simple Decision Tree - A simple decision tree trying to answer the question of whether or not to go on a picnic.

\section{$6.2 .3 \quad \mathrm{~J} 48$}

$\mathrm{J} 48$ is a specific implementation of the $\mathrm{C} 4.5$ algorithm. C4.5 is an algorithm that is used to generate a decision tree given a training set.

\section{Decision Trees}

A decision tree is a simple data structure used to come to a conclusion based on of a number of observations. At each non-terminal node, a question is asked. The answers to the question are represented by the node's outgoing edges. The tree is traversed in this fashion until a terminal node is reached. The terminal node contains the final conclusion. In a classification context, each non-terminal node is labeled with an attribute, each edge is the value (or range of values) for that attribute, and each terminal node is a class. Each attribute can only appear once in the tree. 
Figure 6.3 shows a decision tree that may be generated for the picnic example discussed in Section 6.2.2. Note that once the decision tree is built, reaching a terminal node is fairly trivial.

\section{C4.5 - Decision Tree Induction Algorithm}

C4.5 recursively builds a decision tree by continually splitting the dataset on a single attribute [25]. The splitting attribute is determined by the normalized information gain (Kullback-Leibler divergence) and becomes a node in the tree and the possible values for the attribute become edges. Each subtree is then recursively built using only the data where the splitting attribute takes the value given by the incoming edge. The algorithm has two stopping conditions. First, when all the data has the same class; in which case a single node tree is constructed that contains the class. Secondly, when there are no more attributes or when the information gain from splitting on each attribute is below a threshold. In this case, a single node tree is constructed which contains the plurality class.

\subsubsection{K-Nearest Neighbors}

$k$-Nearest Neighbors (KNN) is a simple and effective classification technique [7]. While training, the classifier remembers the entire training set. During the classification phase, the classifier finds the $k$ nearest neighbors to the query point. The predicted class is simply the plurality of the $k$ nearest neighbors. Figure 6.4 shows an example of $k$-Nearest Neighbors with a simple search space. 


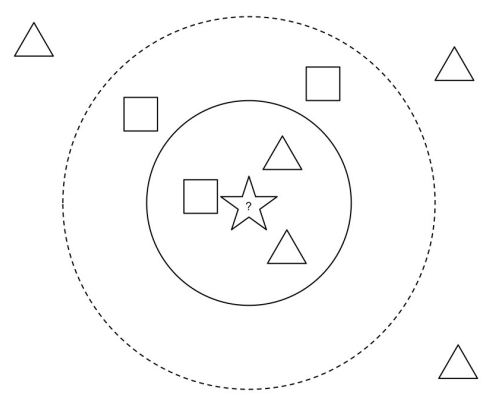

Figure 6.4: K-Nearest Neighbors - A simple example of KNN. If $k=3$, then the query point (the star) will be classified as a triangle. However, if $k=5$ then the query point will be classified as a square.

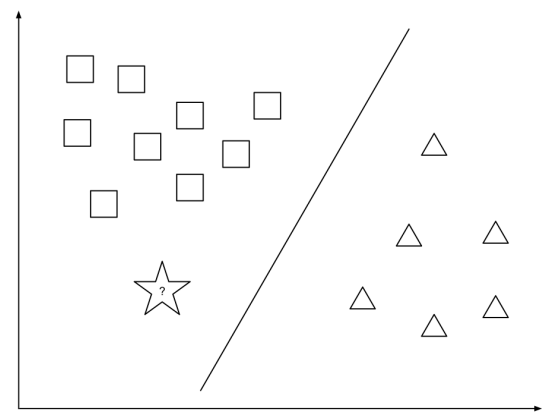

Figure 6.5: Support Vector Machine - A simple example of a support vector machine. The SVM chose a partition that maximizes the margin between the squares and triangles.

\subsubsection{Support Vector Machines}

Support Vector Machines (SVMs) are considered one of the best off-the-shelf classification techniques [4. When training, SVMs use hyperplanes to partition the data into surfaces based on of the different classes of the training examples. When classifying, the SVM finds which surface the query point falls on and give that class to the point. SVMs try and choose the partitioning hyperplane to maximize the margin between the two groups of data. Depending on the implementation, the SVM may choose the optimal partition or just an approximation. 
Figure 6.5 shows a simple example of a linear binary SVM. Note that the partition line is chosen to maximize the distance between the triangles and squares. The query point (the star) falls into the squares' partition and is therefore classified as a square.

\section{Sequential Minimal Optimization}

Sequential Minimal Optimization (SMO) is an efficient algorithm for solving SVMs invented by John Platt in 1998 [22].

\subsubsection{BPNB}

BPNB is a method developed by Chu [3]. It is based on of Naive Bayes, except the relative probability of each feature is accounted for.

BPNB states that the probability of observing class $c$ given document $d$, $\operatorname{Pr}(c \mid d)$, can be represented as:

$$
\operatorname{Pr}(c \mid d)=\operatorname{Pr}(c) \cdot \prod_{i=1}^{n} g\left(\mathrm{f}_{i}, c\right)
$$

Where $g\left(\mathrm{f}_{m}, c\right)$ is the weight of feature $\mathrm{f}_{m}$ in class $c$.

$$
\begin{gathered}
g\left(\mathrm{f}_{m}, c\right)=\beta^{1-\frac{\operatorname{Pr}\left(\mathrm{f}_{m} \mid c\right)}{A v e\left(\mathrm{f}_{m}\right)}}, 0<\beta<1 \\
\operatorname{Ave}\left(\mathrm{f}_{m}\right)=\frac{\sum_{i=1}^{|C|} \operatorname{Pr}\left(\mathrm{f}_{m} \mid c_{i}\right)}{|C|}, c_{i} \in C
\end{gathered}
$$




\subsubsection{WEKA}

SPOONS utilizes several classifiers provided in the WEKA Machine Learning Package. WEKA is an open source package written under the GNU General Public License [9].

\subsection{Singletons}

The SPOONS architecture makes heavy use of singletons to guarantee certain assumptions. A singleton is an object-oriented class that may have at most one instance of itself instantiated at a time. SPOONS uses two types of singletons: singletons that are relative to the base class and singletons that are relative to the child classes.

Base Relative Singletons. Base relative singletons are singletons that only allow one instance of the base class in the inheritance hierarchy to be instantiated at a time. This means that there can only be one instance allowed for the entire inheritance hierarchy. Figure 6.6 shows an inheritance diagram of a hierarchy that uses a base relative singleton. Note that because one of the children has been instantiated, no other class in the hierarchy can be instantiated. 


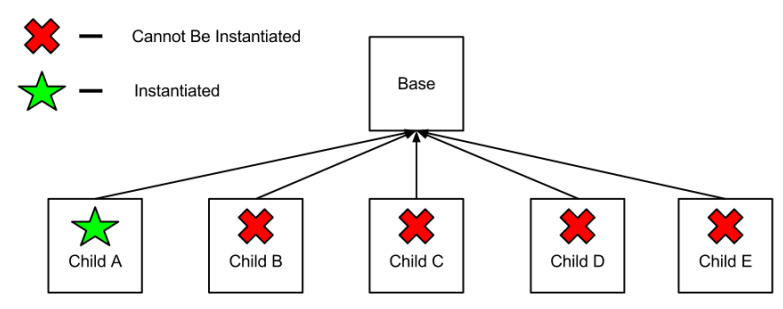

Figure 6.6: Base Relative Singleton - An example base relative singleton inheritance hierarchy. Note that instantiating any child removes the ability to instantiate any other part of the hierarchy.

Child Relative Singletons. Child relative singletons are singletons that allow only one instance of each leaf child in the inheritance hierarchy to be instantiated at a time. This allows the inheritance hierarchy to have as many instances as leaf children. Figure 6.7 shows an inheritance diagram of a hierarchy that uses child relative singletons. Note that all the children can be instantiated once.

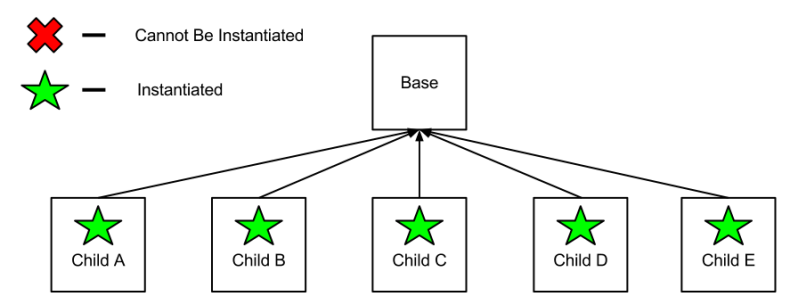

Figure 6.7: Child Relative Singleton - An example child relative singleton inheritance hierarchy. Note that any child can be instantiated, but only once.

\subsection{Accuracy Measures}

The accuracy of classification is primarily determined using three metrics: Recall, Precision, and F Score. 
Consider the situation of trying to classify documents into class $A$ with $A$ and $B$ being the two possible classes. The following definitions are used to calculate the accuracy metrics:

- tp - True Positive. A document with the true class $A$ was correctly classified as $A$.

- fp - False Positive. A document with the true class $B$ was incorrectly classified as $A$.

- fn - False Negative. A document with the true class $A$ was incorrectly classified as $B$.

- tn - True Negative. A document with the true class $B$ was correctly classified as $B$.

\subsubsection{Recall}

The percent of the documents that were correctly classified.

$$
\text { Recall }=\frac{t p}{t p+f n}
$$

\subsubsection{Precision}

The percent of correct classifications of all documents classified as $A$.

$$
\text { Precision }=\frac{t p}{t p+f p}
$$




\subsubsection{F Score}

A harmonic mean between recall and precision. The standard $\mathrm{F}_{1}$ score evenly weighs precision and recall. SPOONS uses the $\mathrm{F}_{0.5}$ score. $\mathrm{F}_{0.5}$ weighs precision more than recall. Precision is being weighed more heavily than recall because every alert that SPOONS generates would require the intervention of a Netflix engineer. Generating too many false positives would just cause SPOONS to be ignored.

$$
\begin{gathered}
F_{\beta}=\left(1+\beta^{2}\right) \cdot \frac{\text { precision } \cdot \text { recall }}{\beta^{2} \cdot \text { precision }+ \text { recall }} \\
F_{0.5}=1.25 \cdot \frac{\text { precision } \cdot \text { recall }}{.25 \cdot \text { precision }+ \text { recall }}
\end{gathered}
$$

\subsubsection{Coverage}

In the context of outage detection, the $\mathrm{F}_{0.5}$ score cannot completely capture the effectiveness of an outage detection method. A flaw in solely relying on the $\mathrm{F}_{0.5}$ score is that an outage detection method can produce an unjustly high F score by generating long alerts. Taken to the extreme, an outage detection method can generate just one alert and have it span the entire evaluation period. This one alert will capture every service outage, and therefore have a recall of 1. Also, the single alert it generates will intersect with a real outage, which will produce a precision of 1 . No matter the type of F score used, a precision and recall of 1 will result in the highest possible $\mathrm{F}$ score of 1 . Figure 6.8 shows a graphical representation of this problem.

To counteract this, an outage detection's coverage is also taken into account. Coverage is the percentage of frames in the evaluation period that are during alerts. 


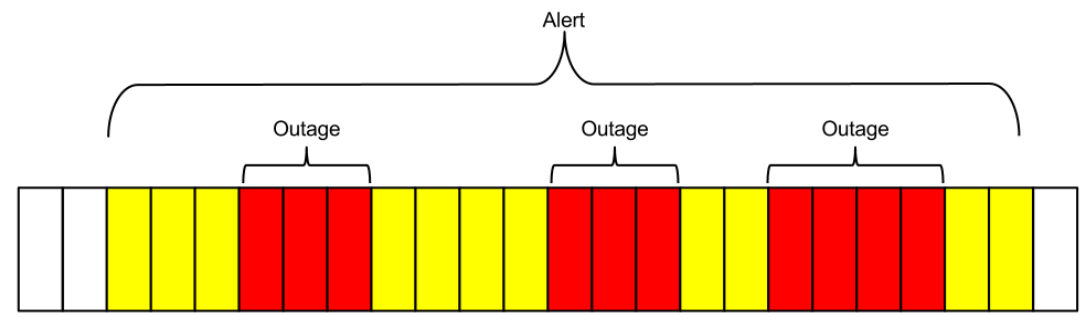

Recall $=3 / 3 \quad$ Precision $=1 / 1 \quad$ Coverage $=21 / 24$

Figure 6.8: Coverage Example - A long alert producing an unjustly high precision and recall. 


\section{Chapter 7}

\section{Twitter API}

All of the data that SPOONS uses is obtained in real time using the Twitter Search REST API [30].

\subsection{Rate Limiting}

Twitter imposes a limit on the number of queries to the Search API. Twitter does not publish the official limit. However, our experiments suggest that SPOONS can query the API for all new Tweets once every two minutes without suffering from rate limiting.

\subsection{Pagination}

Twitter paginates the results from its search API. The maximum results you can get per page is 100 , and each query can return at most 15 pages. Therefore when there are more than 1500 tweets generated per minute, SPOONS must do 
multiple search queries.

\subsection{Query Anatomy}

The typical structure of a Twitter API query is shown in Figure 7.1 .

$$
\begin{aligned}
& \text { http://search.twitter.com/search.json?q= }=\langle\text { query }\rangle \& \mathbf{r p p}=100 \& \\
& \text { result_type=recent\&since_id }=\langle\text { tweet id }\rangle \& \mathbf{m a x} \_\mathbf{i d}=\langle\text { tweet id }\rangle
\end{aligned}
$$

Figure 7.1: Twitter API Query - The structure of a typical query to the Twitter API.

The parameters are:

json: Twitter can supply the result data in either ATOM or JSON format. Testing with both have shown that the ATOM results are less consistent and provide less data. Because of the more accurate information returned from the JSON API, we are able to write more efficient queries. Using the ATOM API, we could query Twitter only once every five minutes; as opposed to every two minutes with the JSON API.

q: The search query. Twitter supports some advanced search features such as conjunction and negation.

rpp: "Results Per Page". Twitter paginates the responses from the Search API. SPOONS always uses the maximum pagination value to decrease the number of requests per hour and lessen the chance of being rate limited.

result_type: Twitter allows users to get results ordered by either relevance or time. Since we want to gather all tweets about our query, we choose to 
get the results ordered by time. In addition, the "since_id" and "max_id" parameters do not work when results are sorted by relevance.

since_id: The id of the oldest tweet that should be returned. This is not a hard limit, but provides a nice starting point.

max_id: The id of the most recent tweet that should be returned. It may seem counter-intuitive to provide a cap on the most recent tweet, when one wants to query for all of the most recent tweets. However when a query's results spans across more than 15 pages, it needs to be broken into a new query restarting at the first page. In this situation, not providing an upper limit includes new tweets outside of the original search scope. This can result in tweets that are forever lost to us.

\subsection{Result Anatomy}

Figure 7.2 shows the result from the query "eriq netflix". Notice that some fields, like the geo field, can be null. Also note that the API incorrectly guessed the language of the tweet as Danish. 


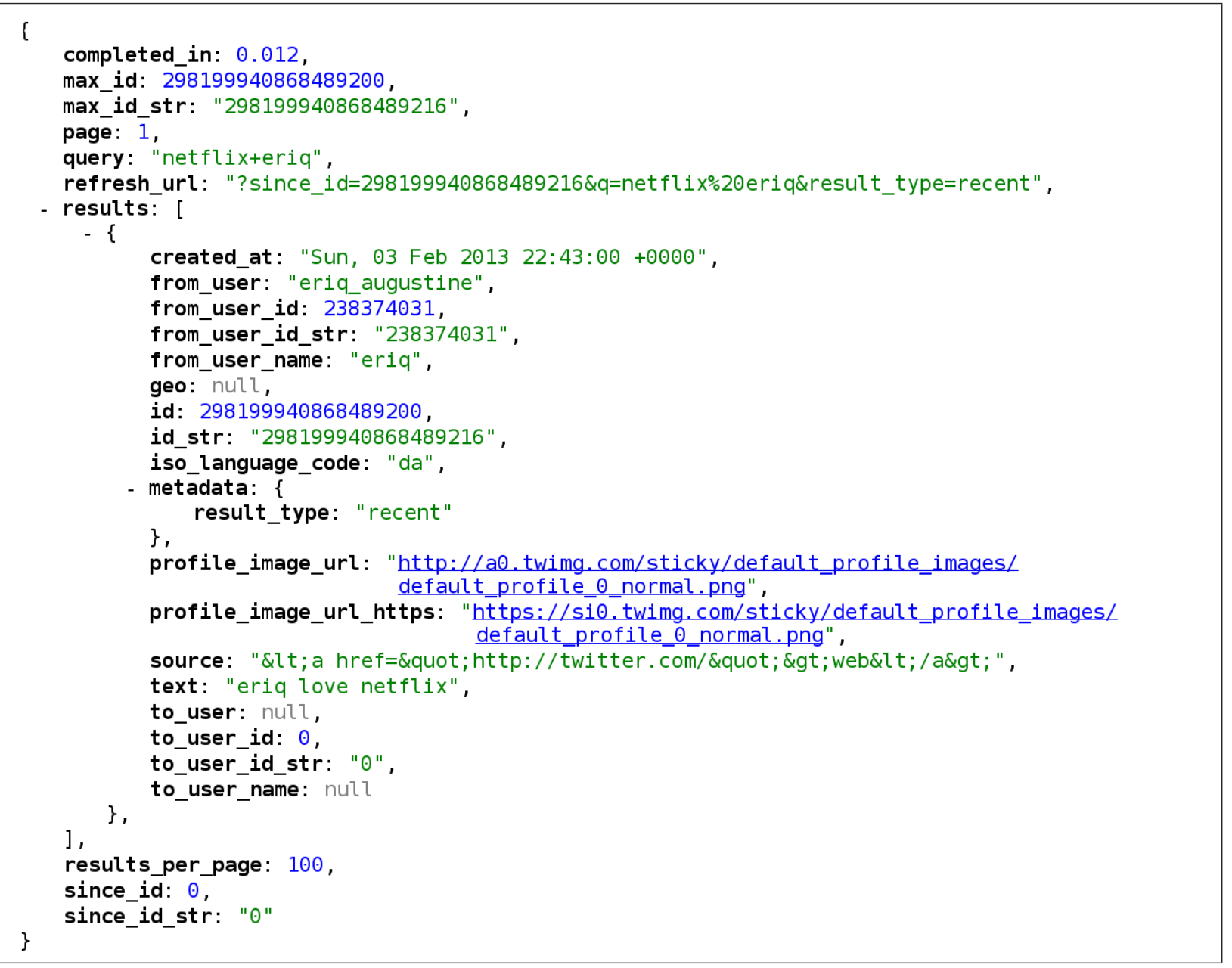

Figure 7.2: Twitter Search API Result - A JSON result from the Twitter Search API 


\section{Part 2}

\section{SPOONS Architecture}




\section{Chapter 8}

\section{Architecture Breakdown}

There are multiple levels of architecture within SPOONS that need to be discussed. Chapter 9 describes the framework architecture (Figure 8.1). The framework architecture describes the relations between the different pieces of the SPOONS framework. Chapter 10 describes both the layout of the different servers involved in the SPOONS system and the Distribution Model which describes how

pieces of work are distributed between the different servers. Finally, Chapter 11 discusses the architecture of the database that backs SPOONS. 


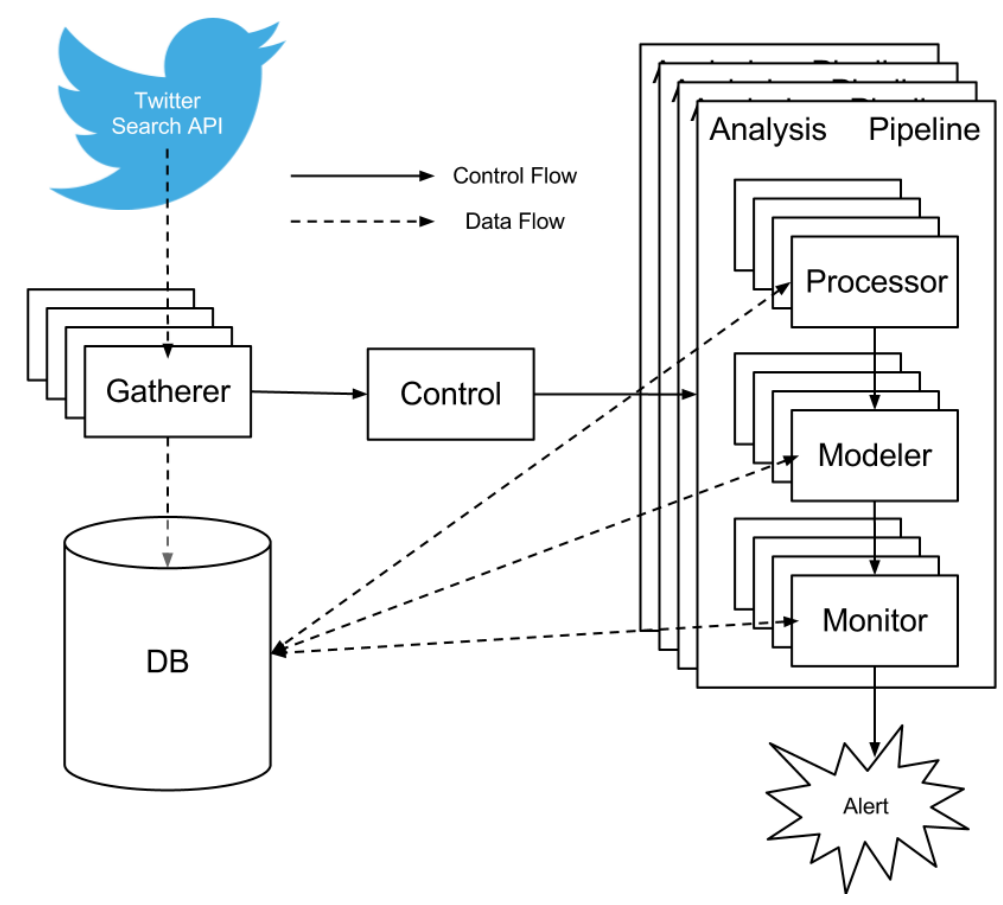

Figure 8.1: SPOONS Framework Architecture - The flow of control and data through the SPOONS framework system.

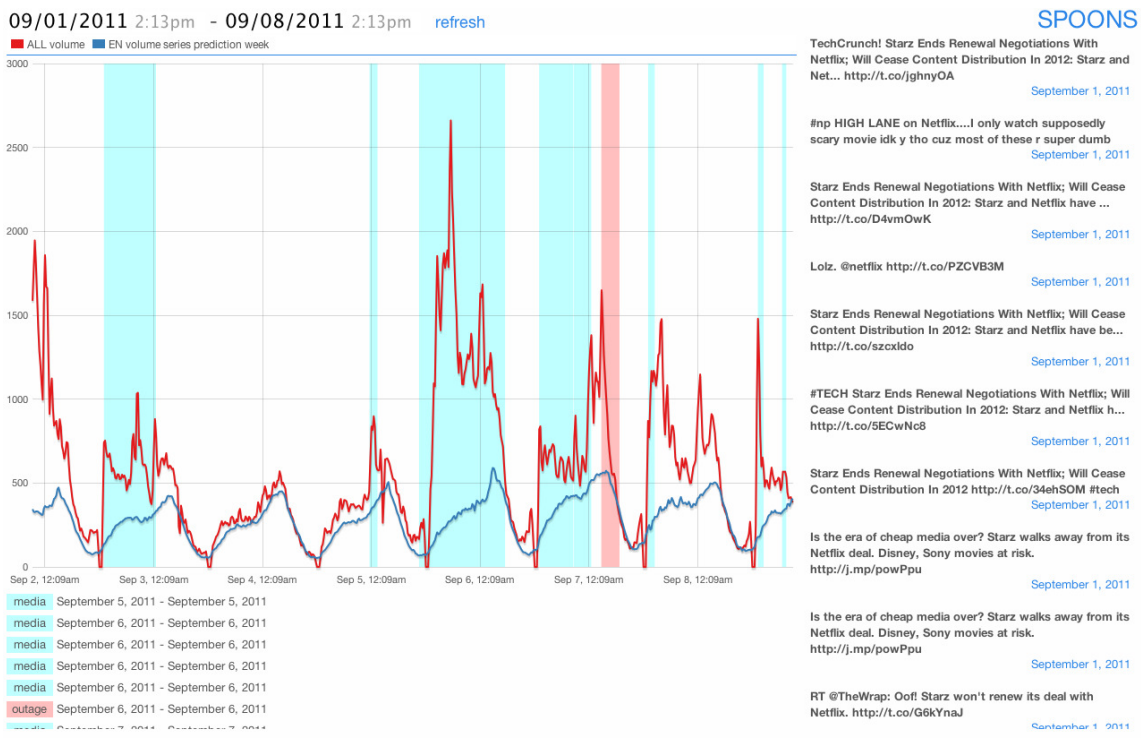

Figure 8.2: SPOONS UI — The web UI for SPOONS. 


\section{Chapter 9}

\section{Framework Architecture}

This chapter describes the architecture of the SPOONS framework. The SPOONS framework includes all pieces of SPOONS that take the data from gathering all the way through to final analysis.

\subsection{High Level Solution}

The general solution taken by SPOONS consists of four main steps:

Collect: Collect tweets from Twitter.

Process: Convert the tweets from plain text to some form of information that can be analyzed.

Model: Use the information generated from the previous step to build a mathematical model of the information. Use past information to predict what the current model of the data should look like.

Compare: Compare the two models generated in the previous step. A significant 
divergence means that there is anomalous traffic.

\subsubsection{Framework Overview}

Figure 8.1 shows the flow of control and data through the SPOONS framework. Data comes into SPOONS in the form of Tweets collected by the Gatherers, and leave SPOONS in the form of alerts generated by the Monitors.

Gatherer. Gatherers are responsible for collecting documents from a specified data source such as the Twitter Search API.

Database. After the tweets are gathered, they are placed in the database. In addition to storing just tweets, the database also stores configuration data, intermediate calculations, and the results of the Analysis Pipelines.

Control. The Control is responsible for controlling the SPOONS server. It maintains data structures with all of the Gatherers and Analysis Pipelines. It is also responsible for communication with other servers in the SPOONS cluster.

Processor. Processors are data transformation utilities that take raw data and puts it in a form that other components can use.

Modeler. Modelers are responsible for building a mathematical model of the data and can be split into two groups: Predictors and Counters. Predictors build a predictive model of the data. Counters build a model of the data that was actually gathered by the system. 
Monitor. Monitors take the models produced by the Predictors and Counters and compares them. The Monitors are responsible for making the final decision about a period of time being anomalous.

\subsection{Gatherers}

The data enters SPOONS at the Gatherers. The Gatherers run periodically (for Twitter, every two minutes). Gatherers are asynchronous and not dependent on any other part of the framework. There may be multiple different Gatherers running on the same machine. Gatherers are abstracted to be able to gather data from any source. Once the Gatherers obtain their data, they place the data in the database and notify the Control that there is new data available to the system.

\subsubsection{Twitter Holes}

It is worth noting that sometimes the Twitter Search API fails to return any data. We have not discovered the cause of this, but Twitter does not report any errors. For unspecified amounts of time the Twitter API reports zero new tweets. We call these dead zones "holes". We have found that a query from a different IP usually does not experience the same hole. To counteract holes, we run Gatherers on multiple servers and resolve duplicate tweets upon insertion into the database. 


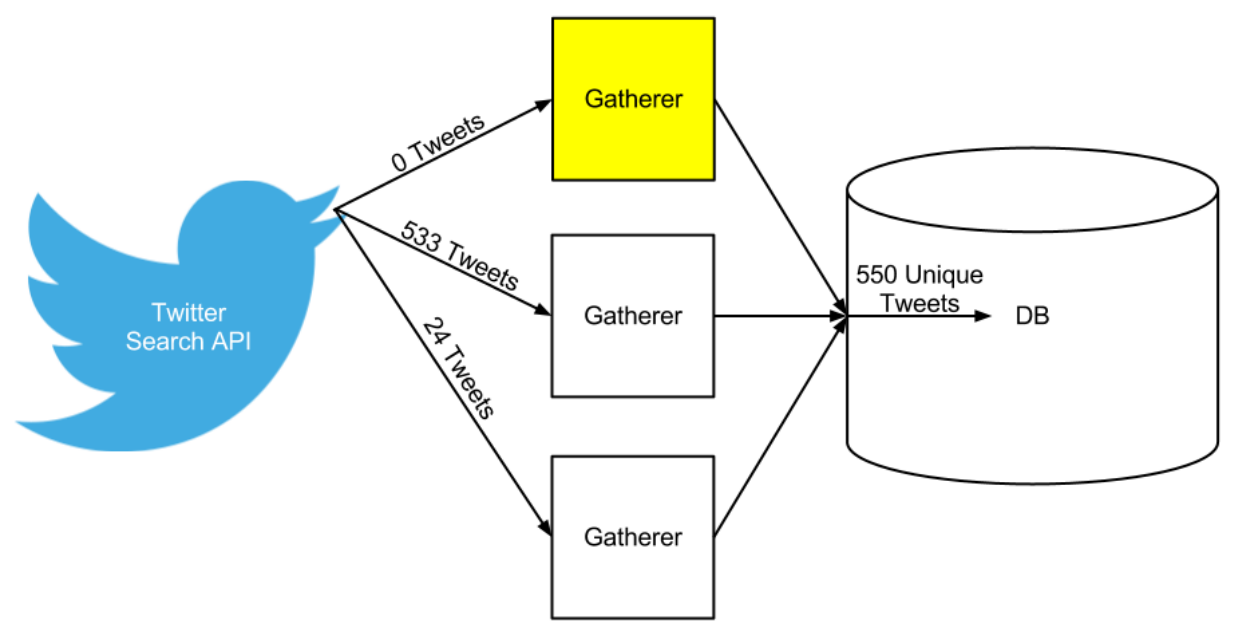

Figure 9.1: Twitter Holes - One server in a hole is covered by two other gathering servers.

\subsection{Processors}

Processors are responsible for processing or transforming data before it goes into the analysis pipelines.

- Classifier Processors: There exists a Processor for every tweet classifier used in SPOONS (see Chapter 12). Because of the high number of classifiers used, these constitute the majority of Processors and form the largest unit of work in SPOONS. These Processors classify every tweet into one of the nine tweet categories discussed in Section 12.3.

- Author Processors: The Author Processors extract the author of tweets and try to establish which authors are credible. These Processors are outside the scope of this work and are discussed in other work [6].

- Valence Processors: The Valence Processors assign a numeric "happiness" score to every tweet. How that score is produced is outside the scope of 
this work (see Section 16.2).

- Document Frequency Processors: The Document Frequency Processors maintain term frequencies and inverse document frequencies for the collection of tweets in SPOONS.

Implementation Notes: Unlike most parts of the analysis pipeline, Processors are a shared resource. That is, multiple analysis pipelines invoke the same Processors. However, it does not make sense to restart the processing once it is started, or to start another instance of the same Processor for the same data. Processors have a finite amount of data to process and may be cumulative. To make sure that no redundant work is done, Processors are singleton. When multiple threads call into a Processor to do work, the Processor blocks all incoming threads until the work is complete. Then, the Processor releases all of the threads that requested the work. This model allows all the analysis pipelines to share the same Processor without any redundancies.

\subsection{Analysis Pipelines}

An Analysis Pipeline (also called Analysis Method) is the analytical center of the SPOONS framework. Pipelines are split into Tasks (see Section 9.5), which are chunked units of work. The exact number and types of Tasks used are different for each pipeline.

The run of an Analysis Pipeline is typically as follows:

1. Pre-process incoming traffic.

2. Model the existing traffic. 
3. Predict what the current traffic should be.

4. Raise an alert if the existing traffic varies significantly from the predicted traffic.

Every Analysis Pipeline gets its own thread, and there is no interdependence between the different pipelines. Currently, SPOONS usually runs more than 20 Analysis Pipelines at a time.

\subsection{Tasks}

Tasks are the core unit of computation in SPOONS. Almost everything that can be "run" is a Task. Every Task gets its own thread, and callers into the Task may request that the Task block the calling thread until the Task is complete.

Implementation Notes: Tasks are singleton with respect to the leaf child class. This ensures that although there are many different Tasks, every Task can be uniquely referenced. This singleton behavior is enforced by checking the fullyqualified class name in the Task base class upon construction. The uniqueness of tasks is very important to SPOONS distribution model discussed in Chapter 10,

\subsection{Modelers}

Modelers are Tasks that are responsible for building a mathematical representation for the data. 


\subsubsection{Predictors}

Predictors build a predictive model of the data. For example, we have noticed that tweet volume tends to be periodic day-to-day and week-to-week. Therefore, a Predictor may model that prediction by guessing that the volume in the future will be the same as it was the previous week or day.

\subsubsection{Counters}

Counters attempt to build a model of data that was actually gathered by the system. Going with the previous example, the Counter for modeling tweet volume would simply count the number of tweets gathered for a period.

\subsection{Monitors}

Monitors take the models produced by the Predictors and Counters and compares them one point at a time. If the two models differ significantly, then an alert is raised. The different types of Monitors are described in detail in Section 13.3. The Monitors are responsible for making the final decision about a period of time being anomalous.

\subsubsection{Auto-Tuning}

Monitors are the most configurable part of the Analysis Pipeline taking anywhere from two to six configurable parameters. To find the best set of parameters, the Monitors can automatically run themselves on a training set and search the space of all possible parameters. They then keep the parameters that result in 
the best score. This process is called "auto-tuning".

\subsubsection{Resistance}

At any given time, a Monitor is either in a normal, non-alerting, state or an alerting state. A Monitor's "resistance" is its tendency not to move into or out of an alerting state. The resistance is the number of normal or abnormal observations it needs to be trigger a state change. Monitors are given resistance because otherwise an outliers could cause a Monitor to rapidly switch between alerting and normal states. There currently are three different methods of observing resistance. The method of resistance as well as the resistance thresholds can also be auto-tuned.

\section{Fighting Resistance}

\begin{tabular}{|c|l|c|}
\hline Parameter & Description & Restrictions \\
\hline A & $\begin{array}{l}\text { The number the counter must reach to enter an } \\
\text { alerting state. }\end{array}$ & $A>0$ \\
\hline $\mathrm{R}$ & $\begin{array}{l}\text { The number the counter must reach to enter a nor- } \\
\text { mal state. }\end{array}$ & $R>0$ \\
\hline
\end{tabular}

Table 9.1: Fighting Resistance Parameters

Fighting resistance counts every time that there is a normal period as a +1 , and every time there is an anomalous period as a -1 . If the counter reaches $-A$, then the Monitor is put into an alerting state. If the counter reaches $R$, then the Monitor is put into a normal state. 


\section{Continuous Resistance}

\begin{tabular}{|c|l|c|}
\hline Parameter & Description & Restrictions \\
\hline A & $\begin{array}{l}\text { The number the counter must reach to enter an } \\
\text { alerting state. }\end{array}$ & $A>0$ \\
\hline $\mathrm{R}$ & $\begin{array}{l}\text { The number the counter must reach to enter a nor- } \\
\text { mal state. }\end{array}$ & $R>0$ \\
\hline
\end{tabular}

Table 9.2: Continuous Resistance Parameters

Continuous resistance must get $A$ continuous anomalous observations to enter an alerting state, and $R$ continuous normal observations to enter a normal state.

\section{Window Resistance}

\begin{tabular}{|c|l|c|}
\hline Parameter & Description & Restrictions \\
\hline W & The window size. & $W>0$ \\
\hline C & $\begin{array}{l}\text { The number of anomalous observations necessary } \\
\text { for an alerting state. }\end{array}$ & $0<C<=W$ \\
\hline
\end{tabular}

Table 9.3: Window Resistance Parameters

Window resistance remembers $W$ previous observations as being normal or anomalous. If the number of anomalous observations is or exceeds $C$, then an alerting state is declared. Otherwise, the Monitor stays in a normal state. 


\subsubsection{Smoothers}

The Monitors have a chance to smooth the data before it gets analyzed. Smoothers take in a stream of data. As with resistance methods, different smoothers and smoothing parameters can be auto-tuned.

\section{No Smoother}

Do not smooth. If this smoother is put into the parameter search space, then the effects of no smoothing can be seen.

\section{Moving Mean Smoother}

\begin{tabular}{|c|c|c|}
\hline Parameter & Description & Restrictions \\
\hline $\mathrm{W}$ & The window size. & $W>0$ \\
\hline
\end{tabular}

Table 9.4: Moving Mean Smoother Parameters

The Moving Mean Smoother works by taking the mean in a sliding window of size $W$. This Smoother tolerates a smaller window if there is not enough data available. Therefore, this Smoother always outputs a number for every number in the input stream.

\subsection{Control}

The Control is the center of a SPOONS instance. It handles the flow of all control and has the ability to start and stop any Task or Analysis Pipeline on 
demand. It holds references to all the threads for the Gatherers and Analysis Pipelines. The Control handles all the setup and tear down in the system.

There are different types of Controls that decide the behavior of SPOONS on each respective server. There are three types of Controls: Master Control, Worker Control, and Single Control. The Control is singleton with respects to the base class. Therefore, only one instance of any type of Control can be active on a server at any given time.

Implementation Notes: The Control is very careful to never allow anyone to own a reference to the currently running Control. All requests to the Control are made statically to the "Control" base class. The base class then forwards the request onto the specific instance of Control currently active on the server. This is done so that the rest of the SPOONS system never knows what kind of Control is currently active. Because of that, a server can be switched between different roles without restarting the system or notifying any other components of the SPOONS system.

\subsubsection{Master Control}

The Master Control is the Control that is responsible for the controlling SPOONS when it is in distributed mode. The Master Control maintains information on all the active worker servers including what Tasks are currently assigned to them.

Implementation Notes: The Master Control maintains "shallow execution" of every pipeline in the system. This means that this control runs each pipeline, 
but then distributes work for each pipeline as the work is generated.

\subsubsection{Worker Control}

Worker Controls do not take any initiative to run any tasks. Instead, they just wait for a Master Control to tell them what to do.

\subsubsection{Single Control}

The Single Control is for a SPOONS instance that wants to run on a single server. 


\section{Chapter 10}

\section{Distributed Computation Model}

As discussed before, SPOONS is a multi-server system (Fig 10.1). The SPOONS system uses the master/worker paradigm with a single master and $N$ workers.

All of the servers share two resources: the primary database and a NoSQL property store. When a master or worker comes online, it inserts an entry for itself into the shared property store. If the new server is a worker, it alerts the current master about its existence, and visa-versa, if the new server is a master. In addition, all workers are required to heartbeat to the master every 15 seconds and the master heartbeats to the workers every 15 seconds. Using this system, the master always knowns about all of the workers and the worker always knows about the current master. When a server misses three heartbeats, the server expecting that heartbeat assumes that the server has gone down. 


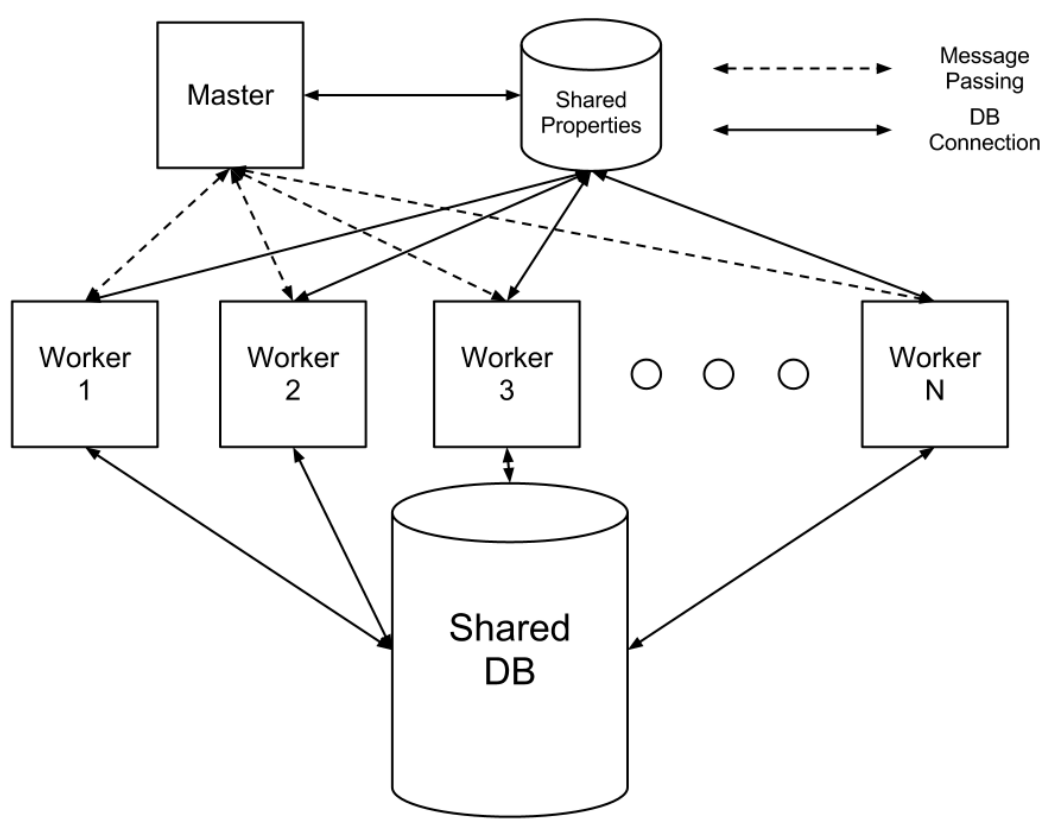

Figure 10.1: SPOONS Server Architecture - The server architecture of the SPOONS system.

\subsection{Distribution Requirements}

The design of the SPOONS distribution model was dominated by two main concerns: performance and usability.

Performance. SPOONS is a real-time system. Any attempt at distribution must not compromise the response time of the system. In addition, SPOONS has to be able to survive a server dying. These two concerns led to three requirements:

- Efficiency: A distributed SPOONS should have comparable response time to a single server SPOONS.

- Fault Tolerance: SPOONS should be able to survive any non-database server failing. 


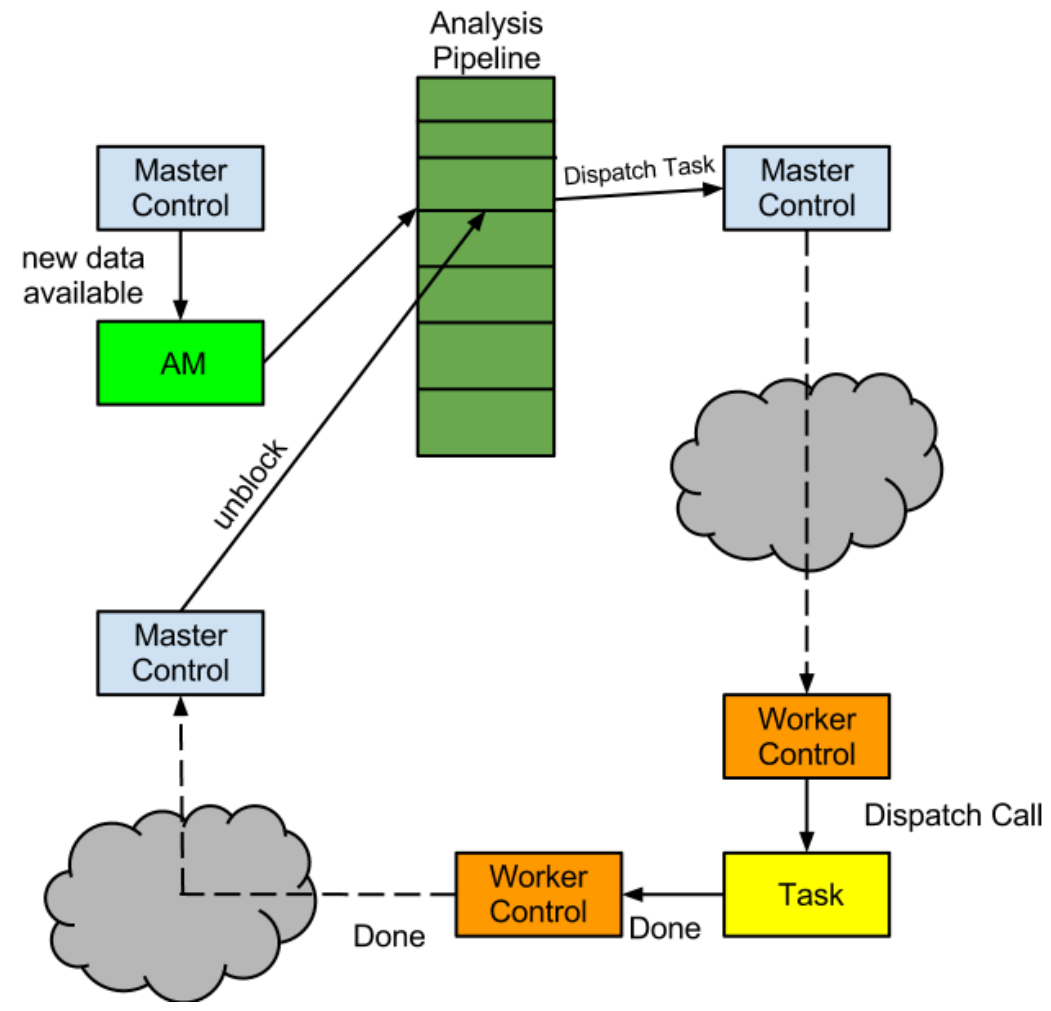

Figure 10.2: SPOONS Distributable Task Flow — The control flow for distributable tasks. 
- Scalability: Scaling a SPOONS cluster should be straightforward.

Usability. SPOONS is meant to be a general real-time analysis system that many different types of people can use. The average developer using SPOONS should be concerned only with the framework level. Server, network, and distribution semantics should remain transparent to a user of the framework. To make a distributed SPOONS easy to use for a developer working with the framework, three requirements are stipulated:

- DB: There should be one database, and a framework developer should not have to do anything that is not required on a simple single-server system.

- Framework Complexity: All distribution specifics should be hidden from the framework developer.

- Single Mode: SPOONS should be able to run on a single server.

\subsection{Distribution Assumptions}

The SPOONS distribution model relies on two assumptions about the system: every server contains exactly the same data in memory and every Task can be uniquely referenced.

\subsubsection{Same Data}

SPOONS assumes that every server has the same data in memory on every server. This means that not only does every server need to have the same data structures in memory, but also that every server needs to have the same classes 
instantiated. The only exception to this assumption is the Control. Depending on the role of the server, a different Control is instantiated. Because of this assumption, we do not have to worry about active replication between servers or a worker being asked to do work that requires a class that is not instantiated.

\subsubsection{Uniquely Referenced Tasks}

As stated in Section 9.5, Tasks are the basic unit of work inside SPOONS. When a worker is told to execute some work, it is being asked to execute a specific Task with specified parameters. Therefore, Tasks need to be able to be referenced by a key that can be serialized and sent over the wire from the master to the worker.

\subsection{Distributable Tasks}

Distributable Task is a subclass of Task that provides the distribution mechanism for Tasks. When a Task is to be distributed, the Distributable Task calls into the Control and requests that the Control distributes it. The next step varies depending on the type of Control that is active:

\subsubsection{Master Control}

The Task distributing control flow is described in Figure 10.2. A Master Control blocks the calling thread and sends a message to a selected worket telling it to run the Task with given parameters. The message that goes to

\footnotetext{
${ }^{1}$ The current scheduling algorithm chooses the worker that has the fewest Tasks currently assigned to it.
} 
the worker just contains the Task's unique identifier and the parameters to the Task's run. When the Task is complete, the Worker Control sends the Task's return status back to the Master Control. When the master receives a message from the worker that the requested Task has completed its run, it resumes the original calling thread and have it return with the return status given by the worker.

\subsubsection{Worker Control}

Worker Controls do not distribute Tasks. Because Tasks are atomic units of work, Tasks are not supposed to call other Tasks. If Task A calls upon a Worker Control to distribute a Task B, then that means that Task A has violated its own atomicity. This is considered a violation of the framework and causes the Worker Control to throw an error.

\subsubsection{Single Control}

Instead of blocking the calling thread like in the Master Control, a Single Control just uses the calling thread to run the Task. When the Task is complete, the Single Control returns control to the caller.

\subsection{Shared Properties}

As previously stated, all servers must maintain a consistent in-memory view of the system. This can be troublesome if a Task needs to maintain cumulative settings or member datum. Not only will this data need to be consistent on all the servers, but it also needs to maintain this data between starts and stop of the 
system. An Analysis Pipeline should be able to be interrupted at any moment, and then restarted later without losing data or its place.

To enforce these restrictions, SPOONS uses a shared property store. The shared property store is a MongoDB server. Whenever a Task needs to store member datum, it places the data in the shared store, making the data available to any server in the cluster. A Task can first be run to completion on Server A and then, when new data is available, run on Server B. Because the Task stores the necessary information in the shared property store, Server B can have all the information gained from the run on Server A and not lose any positional information.

In addition to storing shared properties, the shared property store houses information on every active server. When a server comes online, it queries the property store to find all the other active servers and inserts itself into the store. If a server fails to heartbeat, then the rest of the cluster that is still active removes the entry for that server from the property store. 


\section{Chapter 11}

\section{Database}

SPOONS is backed by a MySQL database. SPOONS currently uses 225 tables and 35 stored procedures. The 225 tables are further divided into six different categories that are used in different stages of the analysis pipeline. In addition to tweets, configuration data, intermediate calculations, analysis results, and final alerting decisions are stored in the database. Keeping all of this data allows the users to look back at any point in time for reference or debugging.

The database uses naming and schema conventions to maintain organization on its tables. The naming and schema conventions allow different components of the Analysis Pipeline to be interchanged without any need to change/reprocess the data. In addition the conventions allows the UI to represent new tables without the need for specifying them. 


\subsection{Tables and Schemas}

Each stage in an analysis pipeline generally stores some information in the database. Because each stage generally deals with similar types of data, these tables are considered to be in the same group. We enforce group membership using hints in the table names. For example, the table name "RESULT_EN_class_heuristic_bayes_net" gives five hints as to the type of the table.

1. RESULT - Marks this table as a result table. This means that it is guaranteed to be shown in the UI.

2. EN - The language of the tweets that were input into this method.

3. class - Indicates that this these results are output from a tweet classifier.

4. heuristic - States that the type of classifier used was a heuristic classifier.

5. bayes_net - The name of the classifier used.

Using all of these hints, the UI can then ask for data for specific types of tables (eg. all result tables that are for English tweets).

The six different top level categories that SPOONS recognizes are:

1. CALC - These tables store intermediate results in analysis pipelines. CALC tables are typically only used when large sets of past data are needed for cumulative models. They are never shown to the UI.

2. CONFIG - Contains information that analysis methods used to configure themselves before runs. These tables have been mostly replaced with the shared property store (see Section 10.4). 
3. DATA - Raw input data. These tables are generally the output from the Gatherers.

4. META - Contains information that is not analyzed, but required by the system. For example, the different classes that the classifiers use along with descriptions of each class.

5. RESULT - These tables are output from some analysis pipeline. They are guaranteed to be shown in the UI. These tables typically contain time series of some analytical signal.

6. TEST - These tables are used for debugging and development. They are never shown in a user-facing UI, however may be shown in development UIs.

The full schemas for select tables are described in Appendix $\mathrm{A}$.

\subsubsection{Data Flow}

The flow of data through the different types of tables is described in Figure 11.1. The data originates from the Gatherers and is moved into DATA tables. Information from DATA, CONFIG, and META tables are analyzed and placed in either CALC, RESULT, or TEST tables. At a later time the data from CALC tables is further analyzed and the results are placed in a RESULT table.

\subsubsection{Tweets Table}

As the most used and important table in the database, the table that houses all of our tweets, "DATA_tweets", gets special attention. 


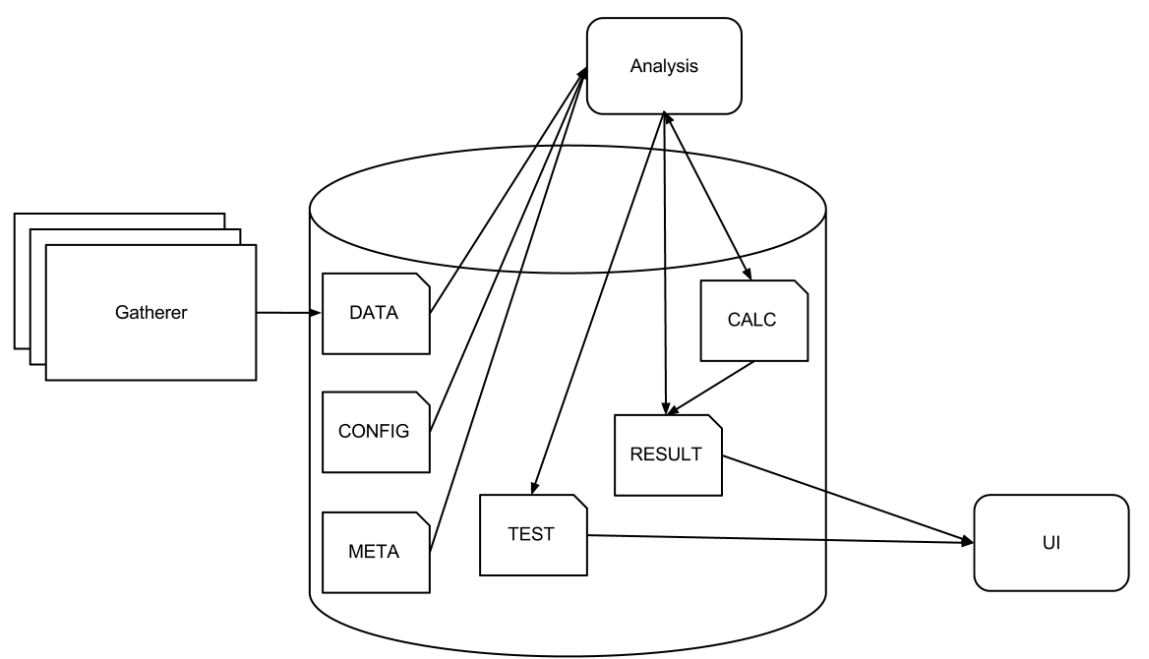

Figure 11.1: Database Data Flow — The flow of data through the different types of tables in SPOONS.

The tweets table contains ten attributes which are described in Table 11.1 .

\section{Frames}

Inside SPOONS, we use a "frame" as the atomic unit of time. Currently, a frame corresponds to a minute. Bucketing the tweets into frames allows us to gain a natural aggregation and smoothing. It also provides a natural index. Maintaining an index on frame_id allows quick retrieval of time series data which is the primary task of SPOONS. Because insertions are generally chronological, insertions are also quick and do not require a rebuild of the B-Tree index [1].

\subsection{UI Stored Procedures}

In addition to utility procedures, the database holds many stored procedures used by the UI. This keeps the UI fairly stable in the face of database changes. 
Data_tweets Schema

\begin{tabular}{|l|l|}
\hline Name & Description \\
\hline id & An auto-incremented primary key. \\
\hline twitter_id & The unique id Twitter gives to a tweet. \\
\hline published & The epoch time that the tweet was posted according to Twitter. \\
\hline content & The raw content of the tweet. \\
\hline source & $\begin{array}{l}\text { Information on where the tweet was posted from (eg. from a third } \\
\text { party app). }\end{array}$ \\
\hline lang & The suggested language of the tweet. \\
\hline author & The author of the tweet. \\
\hline frame_id & The frame that this tweet falls into, has an index on it. \\
\hline place & Information on where the tweet was posted from. This is a JSON \\
& structure and may contain fields such as "city" and "state". \\
\hline geo & Geographical coordinates of place. \\
\hline
\end{tabular}

Table 11.1: Database Tweet Attributes — The database attributes used to describe tweets. 


\begin{tabular}{|l|l|}
\hline Schema Name & Required Columns \\
\hline Volume & start_frame, value \\
\hline Volume Prediction & start_frame, prediction \\
\hline Valence & start_frame, value \\
\hline Valence & start_frame, prediction \\
\hline Class & start_frame, undecided, media, neutral, snafu, watching, response, \\
& complaint, refuse_to_rate, happy \\
\hline Group & start_frame, media, bad, other \\
\hline
\end{tabular}

Table 11.2: Stored Procedure UI Expected Schema - The different types of schemas that the UI looks for in RESULT tables.

\subsubsection{Expected Schemas}

The UI Stored Procedures look for 6 distinct name/schema combinations all of which are required to have the "RESULT" prefix. The different schema requirements are shown in Table 11.2, and described below:

Volume. This schema is for tables that contain time series information about tweet volumes. This includes tables that hold the time series for the total Netflix-related Twitter traffic.

Volume Prediction. These tables contain time series that are predictive models of Netflix-related Twitter traffic.

Valence. These tables contain time series for estimates of the current sentiment about Netflix.

Valence Prediction. These tables contain time series that are predictive models of the sentiment about Netflix. 
Class. These tables contain time series for the volume of tweets that were classified into each of the nine categories described in Section 12.3.

Group. These tables contain time series for the volume of tweets that were classified into each of the thee different groupings described in Section 12.3.1.

The stored procedures further divides the tables by language. The currently recognized languages are English, Spanish, and Portuguese. 
Part 3

Analysis 


\section{Chapter 12}

\section{Classifiers}

\subsection{Why Classification?}

Classification helps discover Netflix service outages by differentiating between different types of Twitter traffic.

Figure 12.1 shows the normal pattern of Netflix-related Twitter traffic over the course of a single week. The peaks appear at around 7pm PST and the valleys are around 2am PST. This kind of pattern is very regular and repeats weekly during normal times. However, where there is some sort of event, the traffic develops spikes. Figure 12.2 shows a period with two anomalous spikes. However, sampling tweets from the different spikes hints that the causes for the two spikes are very different. Figure 12.3 shows tweets sampled from each spike. The left spike is composed mostly of tweets indicating that Netflix is experiencing a service outage. The right spike however, is composed mainly of tweets linking to a news article about Netflix. Therefore, we see that not only service outages generate spikes in Netflix-related Twitter traffic. 


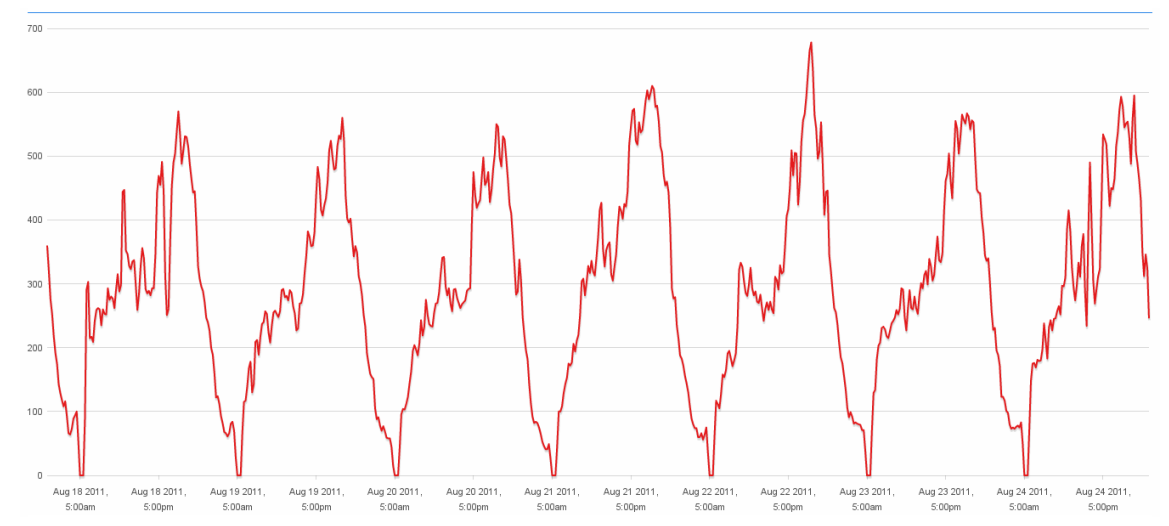

Figure 12.1: Normal Traffic - A week's worth of Netflix-related Twitter traffic. Notice the daily periodicity.

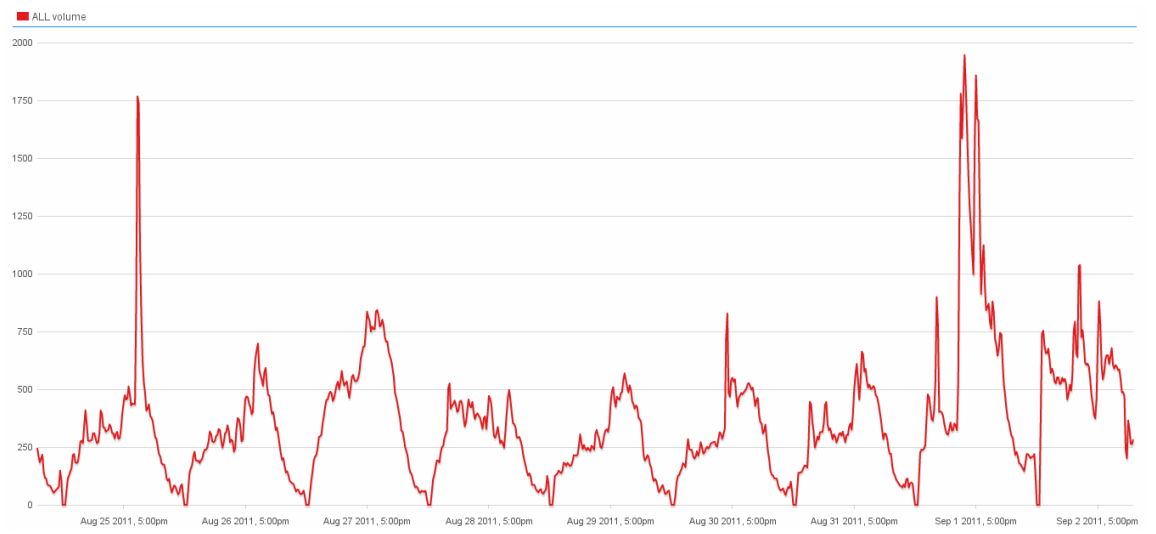

Figure 12.2: Anomalous Traffic - Netflix-related Twitter traffic with two different anomalies.

This is where classifiers become useful. If tweets can be placed into different classes according to their type, then the different types of traffic can be differentiated. Figure 12.4 shows the result of classifying the tweets and then building time series of the classes respective traffic. It becomes obvious that the spike on the left is caused by outage related traffic and that the spike on the right is caused by media related traffic. 


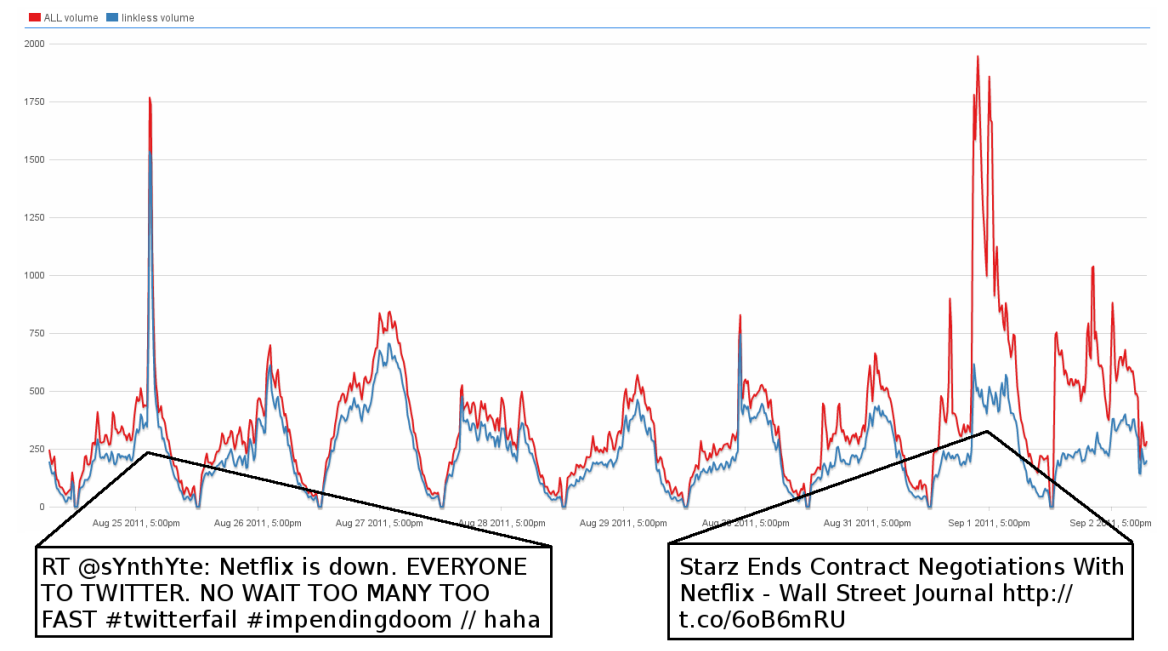

Figure 12.3: Linkless Anomalous Traffic — The same traffic shown in Figure 12.2, with an additional line showing Netflix-related Twitter traffic that does not contain a URL.

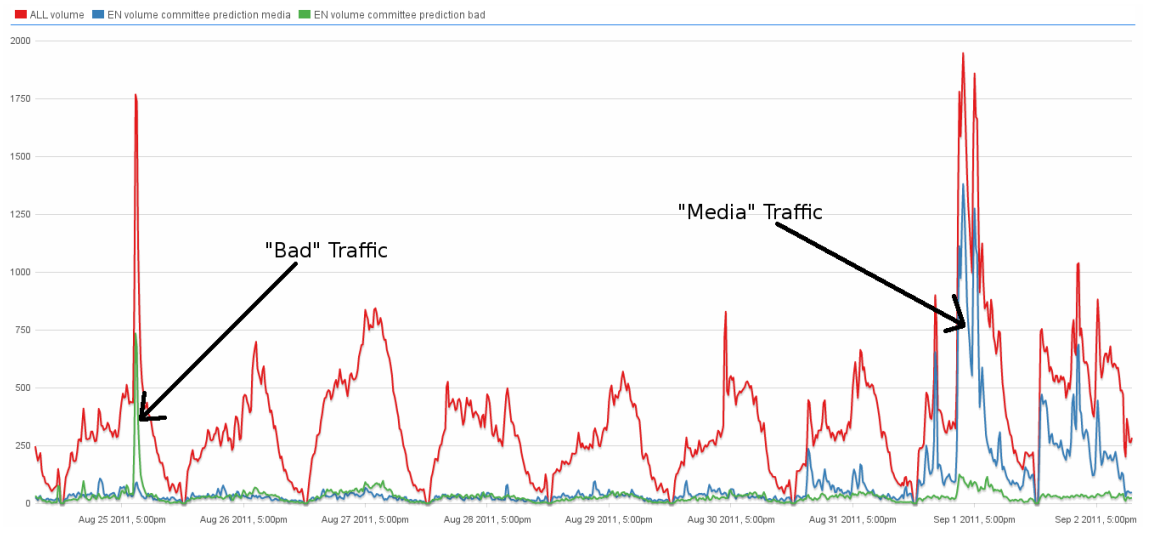

Figure 12.4: Classified Traffic — The same traffic shown in Figure 12.2, with two addition lines: the volume of tweets classified as "Bad" and the volume of tweets classified as "Media". 


\subsection{Classification Roadmap}

The steps that SPOONS takes to use classification to detect service outages can be divided into two categories: prep work and online.

The prep work includes:

1. Observe Netflix-related Twitter traffic and observe the different classes that the tweets fall into.

2. Build a training set biasing anomalous traffic.

3. Classify incoming tweets.

4. Group classified tweets according to the type of traffic that class produces.

5. Establish the best classifiers.

After the off-line prep work is complete, the online analysis can begin:

1. Use the best classifiers in an Analysis Pipeline.

2. Observe the differences between the total traffic and the traffic classified as anomalous.

3. Declare an outage when the two traffics diverge significantly.

\subsection{Tweet Classes}

After observing Netflix-related Twitter traffic, we decided tweets fall into at least one of nine different categories. 
- Media - Tweets relating to a media story about Netflix. Typically a link to a news article.

- Snafu - Tweets that talk about a Netflix outage.

- Complaint - Tweets where people are complaining about Netflix.

- Happy - Tweets that expresses the user's joy about Netflix.

- Neutral - Tweets that are just a neutral observation or comment about Netflix.

- Watching - Tweets that gives updates about what the user is currently watching.

- Response - Tweets that are a neutral response to another user in a Netflixrelated conversation.

- Refuse To Rate - Tweets that we we refuse to rate entirely (usually tweets that are in a different language than the training set).

- Undetermined - This class does not exist in the wild. It is used during classification as default for all tweets that don't match any other class.

Examples of tweets with their corresponding classes are shown in Table 12.1 .

\subsubsection{Tweet Groups}

Because the goal of SPOONS is to detect anomalous traffic, it is useful to collapse the nine classes into three different groups that account for the different types of Netflix-related traffic. 


\begin{tabular}{|l|l|}
\hline Class & Tweet Example \\
\hline Media & $\begin{array}{l}\text { Netflix Now Available Through Facebook - } \\
\text { http://bit.ly/ffpBHH - [Geeky Gadgets] }\end{array}$ \\
\hline Snafu & And netflix is broken. Why is this happening to me. \\
\hline Complaint & $\begin{array}{l}\text { netflix keeps taking little things i like about the } \\
\text { site away...Why? }\end{array}$ \\
\hline Happy & Netflix :) \\
\hline Neutral & about to download this netflix free trial \\
\hline Watching & Watching Family Guy on Netflix \\
\hline Response & netflix list. \\
\hline Refuse To Rate & en serio, QUIERO pagar por algo como Netflix, DEJARME \\
\end{tabular}

Table 12.1: Tweet Class Examples — Examples of the types of tweets that go with each class. 


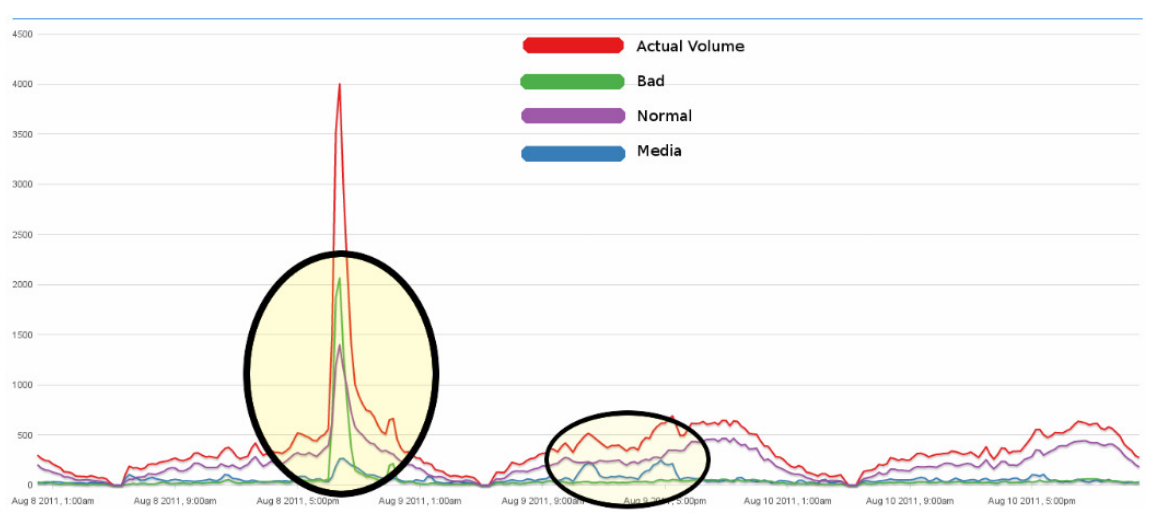

Figure 12.5: SPOONS Groups - The different volumes for different tweet classes during an outage (left) and media event (right).

- Media: Contains only the media class.

- Bad: Contains both the snafu and complaint classes.

- Other/Normal: Contains all other classes.

Figure 12.5 shows the amount of Netflix-related tweets during a Netflix outage and media event. During normal times, the normal traffic is responsible for the majority of the overall traffic. However during outage and media events, we see that the bad and media dominate the respective periods.

\subsection{WEKA Classifiers}

SPOONS uses several classifiers from the WEKA machine learning package [9]. All of these classifiers have been discussed in Section 6.2. The WEKA classifiers used are:

- Naive Bayes

- Bayes Net 
- J48 (a method of generating a C4.5 decision tree [25])

- K-Nearest Neighbors

- SMO (Support Vector Machine trained with Sequential Minimal Optimization [23])

\subsection{Non-WEKA Classifiers}

In addition to the WEKA classifiers, SPOONS uses two classifiers implemented from scratch. Because of low performance and inflexible API, the WEKA classifiers are being reimplemented. As of now, only Naive Bayes has been reimplemented. The other classifier implemented from scratch is a BPNB classifier which is discussed in Section 6.2.6.

\subsection{Text Pre-Processing}

Before the tweets are classified, they are pre-processed. During processing, the text is transformed to make classification of the text easier. Standard text operations like stemming, stopword removal, and case normalization; as well as Twitter and Netflix specific operations like hashtag and movie title recognition are preformed. After the text is processed, it is split into unigrams to be used as features in the classifiers. 


\subsubsection{Text Filtering}

Before the input text is split into features, it goes through heavy pre-processing. The text filtering involves normalizing the case, remove extra characters, and replacing special features.

\section{Link Replacement}

The first step in processing the text is to replace links. Following a link may provide information about a tweet, however the link text of the link itself provides no information. The presence of a link, however, can provide information about a tweet.

\section{Twitter-Specific Symbols}

Tweets often contain several special symbols specific to tweets.

RT. "RT" stands for "re-tweet". It means that the posted tweet is a repost of a tweet made by another user. This symbol contains no reference to the original post. "RT" usually appears at the beginning of the tweet. For example, after the comedian Conan O'Brien posted the following tweet:

If I'm ever a ghost, I hope the person I haunt has Netflix.

There were hundreds of identical tweets that said:

RT: If I'm ever a ghost, I hope the person I haunt has Netflix. 
\#. A "\#" (pronounced "hashtag") is used to mark keywords or topics. Users can search for tweets by hashtag and see the collection of tweets supposedly about the same topic. A hashtag does not have to reference a pre-existing topic.

@. An "@" in Twitter, simply pronounced "at", is a reference to another Twit-

ter user. A reference to a user alerts that user about the posted tweet. For example, the following tweet references my Twitter account.

$\mathrm{Hi}$ there, @eriqaugustine

\section{Emoticon Parsing}

Emoticons are parsed out and replaced with meta words. SPOONS emoticon parser was written by Ryan Hnarakis and Allen Dunlea as part of a project for a graduate Artificial Intelligence course. Emoticons provide a plethora of information about a tweet. Sarcasm aside, an emoticon can indicate the sentiment of an entire tweet.

\section{Title Replacement}

Because our tweets are always about Netflix, a television show and movie streaming service, titles are a common occurrence. However, movie and show titles often contain words that can be detrimental to our analysis. For example, "Death At A Funeral" is the title of a movie, but contains two words that have very negative connotations: "death" and "funeral".

Without title replacement, the following tweet would be very difficult to classify: 


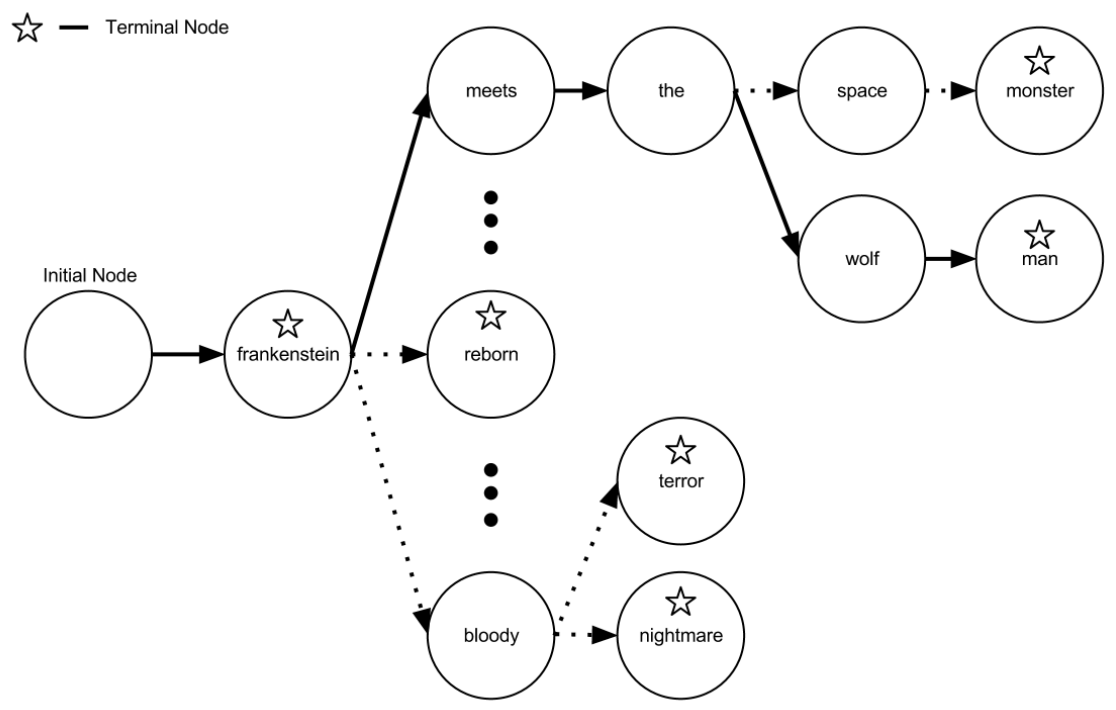

Figure 12.6: Title Trie Walk - A sample trie of Frankenstein movie titles. The solid lines show what nodes the search for "Frankenstein Meets The Wolf Man" would traverse.

\section{Death at a Funeral is hilarious! \#netflix}

However after title replacement, the tweet becomes very easy to classify:

$$
\langle\text { \$title\$ is hilarious! \#netflix }
$$

SPOONS contains a table that has over 50,000 movie and show titles on Netflix. The titles were gathered using the Netflix API. On startup, SPOONS builds a trie (prefix tree) 32 of all of the titles. In this trie titles can only be split on a word level, not on a character level. Therefore, moving to the next node consumes a single word. Searching for a title becomes a simple walk down the trie. If the walk of the trie ends on a terminal node, then a title is found. If not, then the trie is walked again from the beginning starting at the next word in the tweet. Figure 12.6 shows a sample walk in the title trie. 


\section{Stemming}

Stemming finds the root of a word. This allows words to be categorized by their roots which decreases the number of unique words being evaluated and emphasizes linguistic patterns. This preprocessor uses Porter's stemmer for the English language [24].

\section{Stop Word Removal}

Stopwords, or words that carry little or no semantic information, are identified based on a static table of words mapped to levels. Stopwords are assigned levels which allow processes to use different sets of stop words. All words less than 3 character are also automatically considered stop words.

\section{Punctuation/Non-English Character Removal}

Removes all punctuation and characters not in the English alphabet. This simplifies word extraction and comparison.

\section{Meta Words}

Below is an overview of meta words that SPOONS recognizes:

$\langle \$$ title $\$\rangle$ Indicates the presence of a movie or show title.

$\langle \$ \operatorname{link} \$\rangle$ Indicates the presence of a URL.

$\langle$ \$emote:*\$> Replaces an emoticon.

$\langle$ \$RT\$ Indicates that a tweet is a "retweet" (a repeat of another tweet). 
$\langle \$ \# \$\rangle$ Inserted when a "hashtag" is found in a tweet. The original subject of the hashtag is separated off into another word. E.g. "\#Netflix" becomes "〈\$\#\$ Netflix".

$\langle \$ @ \$\rangle$ Inserted when a reference to another Twitter user is made. The user that is the subject of the reference is separated off into another word.

\subsection{Training Set}

The classifiers were trained on a small set of $\mathbf{7 5 9}$ tweets which were pulled from from periods of both normal and anomalous traffic. Each tweet in the training set was manually classified by multiple researchers until consensus about the classification was reached. Because the goal is anomalous traffic detection, the training set over-samples the tweets from media, snafu, and complaint: categories. Table 12.2 documents the structure of the training set and shows the number of tweets classified into each of the eight categories. Tweets were allowed to belong to multiple classes because of posts like, "I love netflix! Watching Law and Order online!", which could be classified as both happy and watching.

See Appendix $\mathrm{D}$ for the full training set.

\subsection{Evaluation}

Each classifier is individually evaluated just on its ability to classify tweets against the training set. Each classifier varies the type of filtering it uses between no filtering and full filtering (every method discussed in Section 12.6.1). 


\begin{tabular}{|l|c|c|c|}
\hline Class & \# Tweets & Class & \# Tweets \\
\hline Media & 103 & Neutral & 66 \\
\hline Outage & 158 & Watching & 135 \\
\hline Complaint & 146 & Response & 30 \\
\hline Happy & 147 & Undetermined & 48 \\
\hline
\end{tabular}

Table 12.2: Netflix-related Twitter Traffic - Overview of the Netflixrelated Twitter post training set used to train classifiers in SPOONS.

Table 12.3 shows the combinations of classifiers and filters used for the classifier evaluation. There will be 24 classifier/filtering combinations.

\begin{tabular}{|c|c|}
\hline Classifier & \\
\hline BayesNetClassifier & \\
\hline BinaryBayesNetClassifier & \\
\hline Binary J48Classifier & \\
\hline BinaryKNNClassifier & \\
\hline BinaryNaiveBayesClassifier & Filtering \\
\hline BinarySMOClassifier & None \\
\hline J48Classifier & Full \\
\hline KNNClassifier & \\
\hline NaiveBayesClassifier & \\
\hline Non-Weka BPNBClassifier & \\
\hline Non-Weka NaiveBayesClassifier & \\
\hline SMOClassifier & \\
\hline
\end{tabular}

Table 12.3: Classifier Evaluation Combinations - The cross product of the classifier and filtering will be used to evaluate the classifiers. 


\subsubsection{Confusion Matrices}

The results of the classification evaluation are shown in confusion matrices. Table 12.4 shows the confusion matrix of the Naive Bayes classifier using full filtering as an example. Down the vertical are the actual classes of the tweets, and across the horizontal are the predicted classes that the classifiers output. Note that the example shows that this classifier incorrectly classified eight media tweets as another class (seen by looking down the media column), and misclassified 22 non-media tweets as media (seen by looking across the row).

Overall accuracy for the classifier can be calculated by taking the number of correctly classified tweets (down the diagonal) over the total number of tweets. Accuracy for a specific classification can be considered two ways: accuracy of classifying tweets from that class and accuracy of classifying into that class. The accuracy of classifying tweets from a class can be seen by looking down a column. The number correctly classified tweets (bold) over the total number of tweets in that column. The accuracy of classifying tweets info a class can be seen using the same method as before, except looking across the class's row instead of its column. 


\begin{tabular}{|c|c|c|c|c|c|c|c|c|}
\hline Classified $>$ & media & neutral & snafu & watching & response & complaint & refuse to rate & happy \\
\hline media & 81 & 3 & 0 & 1 & 3 & 0 & 14 & 1 \\
\hline neutral & 0 & 23 & 3 & 14 & 11 & 11 & 1 & 3 \\
\hline snafu & 0 & 7 & 81 & 8 & 4 & 55 & 2 & 1 \\
\hline watching & 0 & 26 & 0 & 82 & 5 & 12 & 0 & 10 \\
\hline response & 2 & 12 & 3 & 6 & 3 & 1 & 0 & 3 \\
\hline complaint & 1 & 9 & 36 & 9 & 8 & 77 & 3 & 3 \\
\hline refuse to rate & 5 & 4 & 1 & 2 & 4 & 9 & 21 & 2 \\
\hline happy & 0 & 18 & 5 & 22 & 5 & 31 & 1 & 65 \\
\hline
\end{tabular}

Table 12.4: Example Classification Confusion Matrix - An example confusion matrix for a classifier. The undecided class was removed because there were no tweets in that class.

\subsubsection{Results}

Table 12.7 shows a summary of the results of the evaluation. The SMO classifier took the top two spots with a top accuracy of $\mathbf{. 5 7 5 0}$. This is a decent accuracy, but much of the misclassification occurs between classes that don't matter as much when trying to identify the different types of classes. For example, it does not matter if a watching tweet is misclassified as a happy tweet. Both of those classes contribute to normal background traffic. Table 12.5 shows the confusion matrix for the top ranked classifier (SMO with no filtering). All of the misclassification of the other group that would become correct classification if the nine classes were compressed to the three groups are bold. Because of this, the classifiers will be evaluated again, but the different classes will be compressed into their respective groups before the results are evaluated. 


\begin{tabular}{|c|c|c|c|c|c|c|c|c|c|}
\hline Classified & undecided & media & neutral & snafu & watching & response & complaint & refuse to rate & happy \\
\hline undecided & 0 & 0 & 0 & 0 & 0 & 0 & 0 & 0 & 0 \\
\hline media & 0 & 98 & 0 & 0 & 2 & 2 & 0 & 0 & 1 \\
\hline neutral & 0 & 3 & 11 & 2 & $\mathbf{1 6}$ & $\mathbf{7}$ & 7 & 0 & $\mathbf{2 0}$ \\
\hline snafu & 0 & 0 & 1 & 87 & 4 & 4 & 41 & 0 & 21 \\
\hline watching & 0 & 2 & $\mathbf{1 2}$ & 3 & 88 & $\mathbf{5}$ & 4 & 0 & $\mathbf{2 1}$ \\
\hline response & 0 & 2 & $\mathbf{5}$ & 4 & $\mathbf{7}$ & 0 & 3 & $\mathbf{1}$ & $\mathbf{8}$ \\
\hline complaint & 0 & 2 & 5 & 40 & 4 & 1 & 68 & 1 & 25 \\
\hline refuse to rate & 0 & 9 & $\mathbf{1}$ & 1 & $\mathbf{1}$ & $\mathbf{1}$ & 0 & 18 & $\mathbf{1 7}$ \\
\hline happy & 0 & 1 & $\mathbf{9}$ & 3 & $\mathbf{1 3}$ & $\mathbf{6}$ & 8 & 0 & 107 \\
\hline
\end{tabular}

Table 12.5: Uncompressed Misclassification Example - The SMO results with no filtering. Every misclassification that would map to the other group is bold.

\subsubsection{Compressed Results}

Table 12.8 shows a summary of the compressed results. After the classes have been compressed, the SMO classifier is still on top but now with a best accuracy of .8583. Compressing the classes into groups greatly increases the accuracy of the classifiers.

It is important to note that not only is overall accuracy important, but also the precision for the snafu group. If SPOONS is stable capturing and classifying anomalous traffic, then when there is a spike then it will be visible. However if there are too many false positives, then a spike cannot be trusted. Recall affects the size of a spike, but precision affects the shape of the spike.

Table 12.6 shows an example of a compressed classification confusion matrix. The bold cells shows snafu tweets that were misclassified as other groups. Our number one priority is to minimize these cells. These cells represent the precision of the classifier for the snafu group (lower numbers generate higher precision). 
The italics cells shows tweets that were incorrectly classified as snafu. Although we also want to minimize these cells, they are not as important as the bold cells.

\begin{tabular}{|c|c|c|c|}
\hline Classified & media & snafu & other \\
\hline media & 95 & 0 & 8 \\
\hline snafu & 1 & 252 & 51 \\
\hline other & 13 & 77 & 336 \\
\hline
\end{tabular}

Table 12.6: Misclassified Snafu - A sample compressed classification confusion matrix showing misclassified snafu tweets.

Full results for both the uncompressed and compressed evaluation can be found in Appendix B

\begin{tabular}{|l|l|c|}
\hline Classifier & Text Filter & Accuracy \\
\hline SMOClassifier & None & $\mathbf{0 . 5 7 2 6}$ \\
\hline SMOClassifier & Full & 0.5618 \\
\hline BinaryNaiveBayesClassifier & None & 0.5606 \\
\hline NaiveBayesClassifier & None & 0.5462 \\
\hline J48Classifier & Full & 0.5414 \\
\hline BinaryNaiveBayesClassifier & Full & 0.5414 \\
\hline J48Classifier & None & 0.5294 \\
\hline Non-Weka BPNBClassifier & Full & 0.5210 \\
\hline NaiveBayesClassifier & Full & 0.5198 \\
\hline BinarySMOClassifier & Full & 0.5174 \\
\hline BinarySMOClassifier & None & 0.5126 \\
\hline BinaryBayesNetClassifier & Full & 0.4982 \\
\hline BayesNetClassifier & None & 0.4970 \\
\hline Non-Weka BPNBClassifier & None & 0.4958 \\
\hline
\end{tabular}




\begin{tabular}{|l|l|c|}
\hline Classifier & Text Filter & Accuracy \\
\hline BinaryBayesNetClassifier & None & 0.4958 \\
\hline BayesNetClassifier & Full & 0.4646 \\
\hline BinaryJ48Classifier & Full & 0.4622 \\
\hline BinaryJ48Classifier & None & 0.4514 \\
\hline BinaryKNNClassifier & None & 0.4406 \\
\hline KNNClassifier & None & 0.4382 \\
\hline Non-Weka NaiveBayesClassifier & Full & 0.4082 \\
\hline KNNClassifier & Full & 0.3854 \\
\hline BinaryKNNClassifier & Full & 0.3830 \\
\hline Non-Weka NaiveBayesClassifier & None & 0.3553 \\
\hline
\end{tabular}

Table 12.7: Uncompressed Classification Results Summary

\begin{tabular}{|l|l|c|}
\hline Classifier & Text Filter & Accuracy \\
\hline SMOClassifier & Full & $\mathbf{0 . 8 5 8 3}$ \\
\hline SMOClassifier & None & 0.8499 \\
\hline Non-Weka BPNBClassifier & Full & 0.8271 \\
\hline BinarySMOClassifier & None & 0.8259 \\
\hline BinarySMOClassifier & Full & 0.8235 \\
\hline BinaryNaiveBayesClassifier & None & 0.8235 \\
\hline BinaryNaiveBayesClassifier & Full & 0.8199 \\
\hline NaiveBayesClassifier & None & 0.8175 \\
\hline Non-Weka BPNBClassifier & None & 0.8151 \\
\hline J48Classifier & Full & 0.8091 \\
\hline
\end{tabular}




\begin{tabular}{|l|l|c|}
\hline Classifier & Text Filter & Accuracy \\
\hline NaiveBayesClassifier & Full & 0.8079 \\
\hline BinaryKNNClassifier & None & 0.7899 \\
\hline J48Classifier & None & 0.7887 \\
\hline KNNClassifier & None & 0.7863 \\
\hline KNNClassifier & Full & 0.7623 \\
\hline Non-Weka NaiveBayesClassifier & Full & 0.7611 \\
\hline BinaryKNNClassifier & Full & 0.7575 \\
\hline Non-Weka NaiveBayesClassifier & None & 0.7263 \\
\hline BayesNetClassifier & None & 0.7263 \\
\hline BinaryJ48Classifier & None & 0.6879 \\
\hline BinaryBayesNetClassifier & Full & 0.6855 \\
\hline BinaryBayesNetClassifier & None & 0.6819 \\
\hline BinaryJ48Classifier & Full & 0.6759 \\
\hline BayesNetClassifier & Full & 0.6603 \\
\hline
\end{tabular}

Table 12.8: Compressed Classification Results Summary 


\section{Chapter 13}

\section{Outage Detection}

\subsection{Ground Truth}

Netflix has provided us with a list of outages that occurred between March 14, 2011 and January 30, 2012. This list is not comprehensive and some of the times are questionable. Some of the outages contained in the list are internal outages that did not affect their streaming service. These outages generated no signal on Twitter. Therefore, errors of omission could fall into one of two categories: true failures to recognize outages, and uncatchable outages. Regardless, we use this as our ground truth about all of the Netflix outages in that time period.

\subsection{Success Metrics}

Recall from Section 6.4, the definition of True Positive, False Positive, and False Negative. In the context of outage detection, these metrics have more specific definitions: 
- True Positive - Any intersection between a reported outage range and a detected outage range.

- False Positive - Any detected outage that has no intersection in the events reported by Netflix.

- False Negative - An alert that has no intersection on an event reported by Netflix is a false negative.

Precision. Netlfix has specified an acceptable precision of 0.5. This means that SPOONS is allowed to generate an incorrect alert for ever correct alert. Any precision lower than 0.5 will cause SPOONS to be too noisy and consume too much engineer time.

Coverage. The list of outages that Netflix supplied has a coverage of 0.3341 . SPOONS tries to minimize the coverage to supply directed outages with low noise.

\subsubsection{Adjusted Score}

Because of the coverage problem, outage detection methods cannot be compared using just their $\mathrm{F}_{0.5}$ scores. Therefore, they will be compared using an adjusted score that rewards a high $\mathrm{F}_{0.5}$ score and punishes a high coverage.

$$
\text { AdjustedScore }=F_{0.5} \times(1-\text { coverage })
$$




\subsection{Monitors}

SPOONS uses a number of Monitors that use different methods to try and detect anomalies in the traffic. This section describes each Monitor used. Each Monitor described has two tables describing the parameters that can tune this Monitor and the inputs that the Monitor takes. In addition, each Monitor will have a text description along with it.

\subsubsection{Baseline Monitor}

\begin{tabular}{|c|c|c|}
\hline Parameter & Description & Restrictions \\
\hline B & Baseline & $B>0$ \\
\hline
\end{tabular}

Table 13.1: Baseline Monitor Parameters

\begin{tabular}{|c|c|c|}
\hline Input & Description & Restrictions \\
\hline $\mathrm{X}$ & Any time series & - \\
\hline
\end{tabular}

Table 13.2: Baseline Monitor Input Arguments

The Baseline Monitor looks for any value above B, and counts that as anomalous. Ironically, because of its naïveté it also provides a decent baseline for the Monitors.

For example, the Baseline Monitor can be used to monitor the number of tweets classified as bad. The Monitor can be assigned a baseline of 200. Therefore whenever there are more than 200 bad tweets in a period, the Baseline Monitor will alert. 


\subsubsection{Windowed Standard Deviation Monitor}

\begin{tabular}{|c|c|c|}
\hline Parameter & Description & Restrictions \\
\hline W & Window Size & $W>0$ \\
\hline L & Lower Threshold & $L>0$ \\
\hline U & Upper Threshold & $U>=L$ \\
\hline
\end{tabular}

Table 13.3: Windowed Standard Deviation Monitor Parameters

\begin{tabular}{|c|c|c|}
\hline Input & Description & Restrictions \\
\hline $\mathrm{X}$ & Any time series & - \\
\hline
\end{tabular}

\section{Table 13.4: Windowed Standard Deviation Monitor Input Arguments}

The Windowed Standard Deviation Monitor is one of the simplest Monitors. This Monitor takes a $W$ sized window worth of data and uses the standard deviation of the window to find outliers. Any outliers more than $L$ standard deviations away are considered anomalies and counts towards an alert, but are still included in the windowed standard deviation calculation. Any value more than $U$ standard deviations away is considered an anomaly, but not included in the standard deviation calculation. The reason for this is that values above $U$ are extreme outliers.

The calculation for the standard deviation is based on of an iterative approach described in Knuth's "The Art of Computer Programming” [14]. Because Knuth's approach is iterative, it can be modified to calculate for a range of values in an on-line fashion. 
Adding the $k$ th value, $x$, to the window:

$$
\begin{gathered}
\operatorname{Mean}(k)=\frac{\operatorname{Mean}(k-1) *(k-1)-M e a n(k-W)+x}{k} \\
V(k)=(x-M e a n(k)) *(x-\operatorname{Mean}(k-1)) \\
T(k)=T(k-1)-V(k-W)+V(k) \\
\text { WindowStdDev }(k)=\sqrt{\frac{T(K))}{k-1}}
\end{gathered}
$$

For example, the Windowed Standard Deviation Monitor can be used to monitor the number of tweets classified as bad with a lower threshold of 2, an upper threshold of 3 , and a window size of 3 . Table 13.5 shows the process of the Windowed Standard Deviation Monitor at each iteration. Note that iterations 1 - 3 are filling the window and will never alert. Iteration 5 observed a value that was over the lower threshold, so generated an alert. However it as under the upper threshold, so was included in the window. Iteration 6 observed a value that was greater than the upper threshold. Therefore, that value produced an alert and was not included in the window.

\begin{tabular}{|c|c|c|c|c|c|}
\hline Iteration & Window & Mean & Standard Deviation & Observed Point & Alert? \\
\hline 1 & - & - & - & 5 & No \\
\hline 2 & 5 & 5 & 0 & 6 & No \\
\hline 3 & 5,6 & 5.5 & 0.7071 & 3 & No \\
\hline 4 & $5,6,2$ & 4.3333 & 2.0817 & 6 & No \\
\hline 5 & $6,2,6$ & 4.6667 & 2.3094 & 10 & Yes \\
\hline 6 & $2,6,10$ & 6 & 4 & 6 & Yes \\
\hline 6 & $2,6,10$ & 6 & 4 & & No \\
\hline
\end{tabular}

Table 13.5: Windowed Standard Deviation Monitor Example - An example of how the Windowed Standard Deviation Monitor determines when to alert. 


\subsubsection{Weekly Offset Windowed Standard Deviation Mon-}

itor

\begin{tabular}{|c|c|c|}
\hline Parameter & Description & Restrictions \\
\hline W & Window Size & $W>0$ \\
\hline L & Lower Threshold & $L>0$ \\
\hline U & Upper Threshold & $U>=L$ \\
\hline
\end{tabular}

Table 13.6: Weekly Offset Windowed Standard Deviation Monitor Parameters

\begin{tabular}{|c|c|c|}
\hline Input & Description & Restrictions \\
\hline $\mathrm{X}$ & Any time series & - \\
\hline
\end{tabular}

Table 13.7: Weekly Offset Windowed Standard Deviation Monitor Input Arguments

The Weekly Offset Window Standard Deviation Monitor leverages the periodicity of tweet volume. Not only is there a daily pattern in traffic, but there is also an even stronger weekly pattern. The stronger weekly pattern makes sense if one views Netflix-related Twitter traffic as a representative for the number of people currently watching Netflix. People tend to have pattern that they follow, and people are more available on different days of the week (especially Friday).

This Monitor holds a windowed standard deviation for every 30 minute time period with 15 minute offsets for every week. Therefore, values are not compared to the other values around it, but to expected values from previous weeks. This Monitor uses the same tactics as the Windows Standard Deviation Monitor for counting anomalies. 
For example, the Weekly Offset Windowed Standard Deviation Monitor can be used to monitor the number of tweets classified as bad. Unlike the Baseline Monitor, this Monitor allows for both weekly trends and daily variance. Therefore a period of 200 bad tweets may be acceptable on a Friday evening when traffic is typically high, but considered anomalous on a Tuesday morning when traffic is typically low.

\subsubsection{Mean Squared Error Monitor}

\begin{tabular}{|c|c|c|}
\hline Parameter & Description & Restrictions \\
\hline $\mathrm{W}$ & Window Size & $W>0$ \\
\hline $\mathrm{T}$ & Threshold & $T>0$ \\
\hline
\end{tabular}

Table 13.8: Mean Squared Error Monitor Parameters

\begin{tabular}{|c|c|c|}
\hline Input & Description & Restrictions \\
\hline $\mathrm{X}$ & The expected time series & - \\
\hline $\mathrm{Y}$ & The actual time series & $Y(k) \leq X(k)$ \\
\hline
\end{tabular}

Table 13.9: Mean Squared Error Monitor Input Arguments

The Mean Square Error Monitor keeps a windowed mean squared error (MSE). This Monitor requires two sets of input, a set of expected values and a set of actual values. Any value that causes the MSE to go above a certain threshold, $T$, counts towards an anomaly.

Adding the $k$ th value to a full window:

$$
V(k)=(X(k)-Y(k))^{2}
$$




$$
M S E(k)=\frac{M S E(k-1) * W-V(k-W)+V(k)}{W}
$$

For example, the Mean Square Error Monitor can be used to monitor the number of tweets classified as bad by letting $X$ be the actual volume of tweets and $Y$ be the number of total tweets not classified as bad. Table 13.10 shows the process of a Mean Square Error Monitor with a window size of 3 and a threshold of 100 at each iteration. Iteration 5 produces an alert because an MSE over the threshold was observed. An MSE over the threshold means that the number of tweets excluding tweets classified as bad became a poor predictor for the actual traffic of all tweets. This indicates that the traffic of bad tweets is becoming a significant factor to the overall Netflix-related Twitter traffic, which signifies an outage.

\begin{tabular}{|c|c|c|c|c|c|c|}
\hline Iteration & Window (Errors) & Old MSE & Observed X & Observed Y & New MSE & Alert? \\
\hline 1 & - & - & 100 & 95 & 25 & No \\
\hline 2 & 5 & 25 & 105 & 95 & 62.5 & No \\
\hline 3 & 5,10 & 62.5 & 100 & 98 & 43 & No \\
\hline 4 & $5,10,2$ & 43 & 95 & 88 & 51 & No \\
\hline 5 & $10,2,7$ & 51 & 100 & 80 & 151 & Yes \\
\hline
\end{tabular}

Table 13.10: MSE Monitor Example - An example of how the MSE Monitor determines when to alert.

\subsubsection{Ratio Monitor}

\begin{tabular}{|c|c|c|}
\hline Parameter & Description & Restrictions \\
\hline $\mathrm{T}$ & Threshold & $0<T<1$ \\
\hline
\end{tabular}

Table 13.11: Ratio Monitor Parameters 


\begin{tabular}{|c|c|c|}
\hline Input & Description & Restrictions \\
\hline $\mathrm{X}$ & The expected time series & - \\
\hline $\mathrm{Y}$ & The actual time series & $Y(k)<X(k)$ \\
\hline
\end{tabular}

Table 13.12: Ratio Monitor Input Arguments

The Ratio Monitor takes the ratio of the actual value over the expected value for every time period. Whenever the ratio dips under the threshold $T$, then that period counts towards an anomaly. This Monitor may seem simple, but the real challenge lies in picking a proper $X$ and $Y$. If a good approximating time series can be chosen, then the Monitor can be very successful.

For example, the Ratio Monitor can be used with $X$ being the actual volume of tweets and $Y$ being the number of total tweets not classified as bad. Therefore, any divergence in $X$ and $Y$ would be caused by the traffic of tweets classified as bad.

\subsubsection{Class Correlation Monitor}

\begin{tabular}{|c|c|c|}
\hline Parameter & Description & Restrictions \\
\hline $\mathrm{W}$ & Window Size & $W>0$ \\
\hline $\mathrm{T}$ & Threshold & $-1<T<1$ \\
\hline
\end{tabular}

Table 13.13: Class Correlation Monitor Parameters 


\begin{tabular}{|c|c|c|}
\hline Input & Description & Restrictions \\
\hline $\mathrm{X}$ & The expected time series & - \\
\hline $\mathrm{Y}$ & The actual time series & $Y(k)<X(k)$ \\
\hline
\end{tabular}

\section{Table 13.14: Class Correlation Monitor Input Arguments}

The Correlation Monitor takes the Pearson Correlation between $X$ and $Y$ for a running window of size $W$. Pearson Correlation is used because of its ability to catch the linear correlation between two time series within a normalized range.

For performance reasons SPOONS uses an approximation of Pearson Correlation which uses the windowed standard deviation approach described in Section 13.3 .2 ,

Adding the $k$ th value, to the window:

Let $\bar{X}$ be the windowed mean of $X$.

Let $\bar{Y}$ be the windowed mean of $Y$.

$$
\begin{aligned}
& T(k)= \\
& \quad T(k-1)+(X(k) * Y(k))-(X(k-W) * Y(k-W)) \\
& \text { Pearson }(k)= \\
& \quad \frac{T(k)-(W * \bar{X} * \bar{Y})}{(W-1) * W i n d o w S t d D e v(X) * W i n d o w S t d D e v(Y)}
\end{aligned}
$$

For example, the Correlation Monitor can use the same tactic described by the MSE and Ratio Monitors, By letting $X$ be the actual volume of tweets and $Y$ be the number of total tweets not classified as bad, the Correlation Monitor can view the effects of tweets classified as bad. 


\subsection{Evaluation}

The classifiers chosen to be used in the outage detection evaluation are the top ten classifiers from the classifier evaluation (see Section 12.8.2). Each of the ten classifiers is paired with one of the monitors six monitors discussed in Section 13.3 making 60 different combinations as shown in Table 13.15 .

\begin{tabular}{|l|l|}
\hline Classifiers & Filtering \\
\hline SMO & Full \\
\hline SMO & None \\
\hline BPNB & Full \\
\hline BinarySMO & None \\
\hline BinarySMO & None \\
\hline BinaryNaiveBayes & None \\
\hline BinaryNaiveBayes & Full \\
\hline NaiveBayes & None \\
\hline BPNB & None \\
\hline J48 & Full \\
\hline
\end{tabular}

\begin{tabular}{|l|}
\hline Monitor \\
\hline Baseline \\
\hline Windowed Standard Deviation \\
\hline Weekly Offset Windowed Standard Deviation \\
\hline Mean Squared Error \\
\hline Ratio \\
\hline Class Correlation \\
\hline
\end{tabular}

Table 13.15: Outage Detection Evaluation Combinations - The cross product of the classifier and Monitor will be used to evaluate the outage detection methods.

After the combination is chosen, the classifier will be run on all of the tweets in the evaluation period. The Monitor will then use the classified data to auto-tune (Section 9.7.1). The output will be a set of optimal parameters and a confusion matrix with the actual results. Table 13.16 shows an example of the J48 classifier paired with the Weekly Window Standard Deviation Monitor. Note that the confusion matrix is much simpler than the classification confusion matrix. The "True" label indicates an outage. It does not make sense to fill the true negative 
(false/false) cell because the confusion matrix is dealing with outages and that cell represents the number of non-outages that were correctly classified as nonoutages. Since neither precision nor recall uses this cell, it can be left undefined.

\begin{tabular}{|c|c|c|c|c|}
\hline Parameter & Value & \multirow{4}{*}{ Predicted Actual } & \multirow{4}{*}{ True } & \multirow{4}{*}{ False } \\
\hline Resistance Method & \multirow{2}{*}{$\begin{array}{c}\text { Fighting } \\
10\end{array}$} & & & \\
\hline Alert Resistance & & & & \\
\hline Recovery Resistance & 6 & & & \\
\hline Smoothing Method & None & True & 84 & 4 \\
\hline Lower Tolerance & 1.0 & False & 120 & $\mathrm{X}$ \\
\hline Upper Tolerance & 6.0 & & & \\
\hline Window Size & 10 & & & \\
\hline
\end{tabular}

Table 13.16: Example Outage Detection Results - An example of the output for an outage detection method.

\subsection{Results}

Table 13.17 shows a summary of the outage detection evaluation results. The full outage detection results are in Appendix [C]

The best outage detection method was the SMO classifier using full filtering and the Weekly Window Standard Deviation Monitor. This method achieved a precision of 0.9583 , a recall of 0.4510 , a $\mathrm{F}_{0.5}$ score of 0.6970 , a coverage 0.2890 , and an adjusted score of 0.4956 . The very high precision means that this method will almost never give an erroneous alert. The recall of this method is only 0.4510 , however this is fairly good considering that not all reported outages produce signals on Twitter.

All of the top ten methods use the Weekly Window Standard Deviation Mon- 
itor. This shows that the weekly trends in Twitter are very strong. The Monitor that took best advantage of the natural trends in the data did the best.

Full filtering was used in five of the top ten methods, which all have a very close adjusted score. This indicates that the type of filtering is not actually significant.

All of the top 35 methods were able to achieve Netflix's requirement of a precision greater than 0.5 . Only 10 of all 50 methods failed to meet this requirement.

\begin{tabular}{|c|c|c|c|c|c|c|c|}
\hline Monitor & Classifier & Filtering & Precision & Recall & $\mathrm{F}_{0.5}$ Score & Coverage & Adjusted Score \\
\hline WeeklyWindowStdDev & SMO & Full & 0.9583 & 0.4510 & 0.6970 & 0.2890 & 0.4956 \\
\hline WeeklyWindowStdDev & BinaryNaiveBayes & Full & 0.8846 & 0.4510 & 0.6699 & 0.2618 & 0.4945 \\
\hline WeeklyWindowStdDev & $\mathrm{J} 48$ & Full & 0.9545 & 0.4118 & 0.6632 & 0.2583 & 0.4919 \\
\hline WeeklyWindowStdDev & $\mathrm{BPNB}$ & None & 0.9667 & 0.4265 & 0.6797 & 0.2894 & 0.4830 \\
\hline WeeklyWindowStdDev & BinarySMO & None & 0.9588 & 0.4559 & 0.7010 & 0.3116 & 0.4825 \\
\hline WeeklyWindowStdDev & SMO & None & 0.9659 & 0.4167 & 0.6711 & 0.2837 & 0.4807 \\
\hline WeeklyWindowStdDev & BinaryNaiveBayes & None & 0.9625 & 0.3775 & 0.6346 & 0.2462 & 0.4784 \\
\hline WeeklyWindowStdDev & NaiveBayes & None & 0.9205 & 0.3971 & 0.6395 & 0.2529 & 0.4777 \\
\hline WeeklyWindowStdDev & BinarySMO & Full & 0.8481 & 0.3284 & 0.5552 & 0.1542 & 0.4697 \\
\hline WeeklyWindowStdDev & $\mathrm{BPNB}$ & Full & 0.9266 & 0.4951 & 0.7180 & 0.3509 & 0.4661 \\
\hline Baseline & BinarySMO & Full & 0.8947 & 0.3333 & 0.5730 & 0.2019 & 0.4573 \\
\hline Ratio & $\mathrm{J} 48$ & Full & 0.9438 & 0.4118 & 0.6597 & 0.3201 & 0.4485 \\
\hline MSE & BinaryNaiveBayes & None & 0.9722 & 0.3431 & 0.6034 & 0.2579 & 0.4478 \\
\hline MSE & NaiveBayes & None & 0.8646 & 0.4069 & 0.6288 & 0.2949 & 0.4434 \\
\hline Baseline & $\mathrm{J} 48$ & Full & 0.9508 & 0.2843 & 0.5337 & 0.1727 & 0.4416 \\
\hline Baseline & SMO & None & 0.8767 & 0.3137 & 0.5486 & 0.2056 & 0.4358 \\
\hline Baseline & BinaryNaiveBayes & Full & 0.9067 & 0.3333 & 0.5763 & 0.2448 & 0.4352 \\
\hline Baseline & NaiveBayes & None & 0.8447 & 0.4265 & 0.6366 & 0.3213 & 0.4321 \\
\hline Baseline & BinaryNaiveBayes & None & 0.9000 & 0.3971 & 0.6328 & 0.3184 & 0.4313 \\
\hline MSE & BinarySMO & None & 0.7982 & 0.4461 & 0.6319 & 0.3193 & 0.4302 \\
\hline MSE & BinarySMO & Full & 0.8367 & 0.4020 & 0.6150 & 0.3032 & 0.4286 \\
\hline
\end{tabular}




\begin{tabular}{|c|c|c|c|c|c|c|c|}
\hline Monitor & Classifier & Filtering & Precision & Recall & $\mathrm{F}_{0.5}$ Score & Coverage & Adjusted Score \\
\hline Baseline & BinarySMO & None & 0.7524 & 0.3873 & 0.5725 & 0.2526 & 0.4279 \\
\hline MSE & $\mathrm{SMO}$ & None & 0.8800 & 0.4314 & 0.6535 & 0.3482 & 0.4259 \\
\hline Baseline & BPNB & Full & 0.9041 & 0.3235 & 0.5657 & 0.2486 & 0.4251 \\
\hline Ratio & BPNB & Full & 0.8947 & 0.3333 & 0.5730 & 0.2607 & 0.4237 \\
\hline Baseline & SMO & Full & 0.8526 & 0.3971 & 0.6168 & 0.3166 & 0.4215 \\
\hline Ratio & $\mathrm{SMO}$ & Full & 0.6600 & 0.3235 & 0.4901 & 0.1430 & 0.4200 \\
\hline Baseline & $\mathrm{BPNB}$ & None & 0.8989 & 0.3922 & 0.6283 & 0.3345 & 0.4181 \\
\hline Ratio & $\mathrm{SMO}$ & None & 0.7250 & 0.2843 & 0.4780 & 0.1420 & 0.4101 \\
\hline Ratio & BinaryNaiveBayes & None & 0.5922 & 0.2990 & 0.4463 & 0.0912 & 0.4056 \\
\hline Ratio & NaiveBayes & None & 0.7931 & 0.2255 & 0.4313 & 0.0627 & 0.4042 \\
\hline Ratio & BinarySMO & Full & 0.5980 & 0.2990 & 0.4485 & 0.1081 & 0.4001 \\
\hline Correlation & $\mathrm{SMO}$ & Full & 0.5636 & 0.3039 & 0.4387 & 0.1097 & 0.3906 \\
\hline Ratio & BinarySMO & None & 0.7333 & 0.2157 & 0.4074 & 0.0533 & 0.3857 \\
\hline Ratio & $\mathrm{BPNB}$ & None & 0.5124 & 0.3039 & 0.4170 & 0.1134 & 0.3697 \\
\hline Correlation & BPNB & Full & 0.5500 & 0.2696 & 0.4084 & 0.0978 & 0.3685 \\
\hline Ratio & BinaryNaiveBayes & Full & 0.8660 & 0.4118 & 0.6332 & 0.4423 & 0.3531 \\
\hline Correlation & BPNB & None & 0.5732 & 0.2304 & 0.3832 & 0.0828 & 0.3514 \\
\hline Correlation & BinaryNaiveBayes & Full & 0.5312 & 0.2500 & 0.3864 & 0.0921 & 0.3508 \\
\hline Correlation & $\mathrm{J} 48$ & Full & 0.6129 & 0.1863 & 0.3476 & 0.0553 & 0.3284 \\
\hline Correlation & $\mathrm{SMO}$ & None & 0.6275 & 0.1569 & 0.3137 & 0.0480 & 0.2987 \\
\hline MSE & $\mathrm{J} 48$ & Full & 0.4444 & 0.6471 & 0.4962 & 0.4363 & 0.2798 \\
\hline MSE & BPNB & None & 0.4518 & 0.6667 & 0.5062 & 0.4683 & 0.2691 \\
\hline Correlation & BinaryNaiveBayes & None & 0.5273 & 0.1422 & 0.2771 & 0.0518 & 0.2627 \\
\hline Correlation & BinarySMO & Full & 0.4507 & 0.1569 & 0.2775 & 0.0650 & 0.2594 \\
\hline Correlation & BinarySMO & None & 0.4384 & 0.1569 & 0.2743 & 0.0661 & 0.2562 \\
\hline Correlation & NaiveBayes & None & 0.6471 & 0.1078 & 0.2426 & 0.0234 & 0.2370 \\
\hline MSE & $\mathrm{SMO}$ & Full & 0.5260 & 0.7941 & 0.5927 & 0.5986 & 0.2379 \\
\hline MSE & BinaryNaiveBayes & Full & 0.5000 & 0.7500 & 0.5625 & 0.5779 & 0.2374 \\
\hline MSE & $\mathrm{BPNB}$ & Full & 0.5327 & 0.7990 & 0.5993 & 0.6247 & 0.2249 \\
\hline WindowStdDev & BinarySMO & None & 0.7029 & 0.9510 & 0.7698 & 0.8537 & 0.1126 \\
\hline
\end{tabular}




\begin{tabular}{|l|l|l|l|l|c|c|c|}
\hline Monitor & Classifier & Filtering & Precision & Recall & $\mathrm{F}_{0.5}$ Score & Coverage & Adjusted Score \\
\hline WindowStdDev & NaiveBayes & None & 0.7029 & 0.9510 & 0.7698 & 0.8576 & 0.1096 \\
\hline WindowStdDev & BinaryNaiveBayes & None & 0.7055 & 0.9510 & 0.7719 & 0.8582 & 0.1094 \\
\hline WindowStdDev & BinarySMO & Full & 0.7029 & 0.9510 & 0.7698 & 0.8591 & 0.1084 \\
\hline WindowStdDev & SMO & None & 0.7055 & 0.9510 & 0.7719 & 0.8610 & 0.1073 \\
\hline WindowStdDev & J48 & Full & 0.7106 & 0.9510 & 0.7760 & 0.8622 & 0.1069 \\
\hline WindowStdDev & BPNB & None & 0.7055 & 0.9510 & 0.7719 & 0.8616 & 0.1068 \\
\hline WindowStdDev & BPNB & Full & 0.7091 & 0.9559 & 0.7759 & 0.8670 & 0.1032 \\
\hline WindowStdDev & BinaryNaiveBayes & Full & 0.7159 & 0.9510 & 0.7802 & 0.8691 & 0.1021 \\
\hline WindowStdDev & SMO & Full & 0.7132 & 0.9510 & 0.7781 & 0.8689 & 0.1020 \\
\hline
\end{tabular}

Table 13.17: Outage Detection Results Summary 


\section{Part 4}

\section{Conclusions}




\section{Chapter 14}

\section{Conclusions}

SPOONS has proven to be a system that is capable of Netflix outage detection using only tweets. This work has proven that all of the contributions stated in Chapter 5 have been fulfilled. Chapters 8, 9, and 10 discuss the design and implementation of the SPOONS system, framework, server architecture, and distributed computation model. Chapter 11 discusses the design of the SPOONS database. Chapters 12 and 13 discuss the design and implementation of the classification based outage detection methods.

\subsection{The SPOONS System}

This work has covered the details of SPOONS as a robust, distributed system. SPOONS has the ability to function on a single server, or scale to as many servers as desired. SPOONS is fault tolerant and can survive nodes dropping out and coming back into the cluster. The current iteration of the SPOONS system has been running for over a year without any major disruptions. 


\subsection{Tweet Classification}

Tweet classification has proven to be a difficult problem (see Section 6.1.1). However, SPOONS has shown that it is possible to classify tweets with high accuracy (0.8583). With knowledge about the domain of the tweets and a tight restriction on the classes, tweets can be classified with high accuracy. Section 12.8 .2 highlights the success of the classifiers.

\subsection{Outage Detection}

SPOONS has shown the ability to detect Netflix service outages with very high precision (0.9583), good recall (0.4510), and with an acceptable coverage (0.2890). This proves that it is possible to use social media to precisely detect Netflix service outages. Section 13.5 highlights the success of the outage detection methods.

\subsection{Fulfilled Requirements}

Netflix provided six requirement that SPOONS was to fulfil (see Chapter 4):

1. Structural Independence

2. Use of Amazon Web Services

3. Real-Time Detection

4. Precise Outage Detection

5. Comprehensive Outage Detection 
6. User-Friendly UI

The scope of this work covers the first five of these requirements. The UI for SPOONS was built by Matthew Tognetti as his senior project.

Structural Independence. SPOONS was build from scratch and uses no infrastructure from Netflix.

Use of Amazon Web Services. SPOONS is deployed on Amazon Web Services.

Real-Time Detection. SPOONS is a real-time system that continuously runs and provides results within minutes.

Precise Outage Detection. Section 13.5 shows that the top 35 outage detection methods provide the precision required by Netflix. The top method provides almost perfect precision.

Comprehensive Outage Detection. Although the best outage detection methods provide a recall greater than 0.4 , the true comprehension of a method is beyond the scope of this work. Accurately determining how comprehensive the outage detection methods are would require an omniscient way of determining when Twitter generates an outage signal. A more accurate list of Netflix outages can also serve as a way to test if the outage detection methods are comprehensive. 


\section{Chapter 15}

\section{Current Limitations of SPOONS}

Real Time Tuning. All the results generated were the result of auto-tuning on the entire set of data. This assumes that the traffic that occurs in the future will be similar to the traffic that occurred in the past. Ideally, SPOONS would continually re-tune itself every time new outage data is acquired.

Severity. SPOONS does not try to determine how severe an outage is. Severity can be measured in two different ways: breadth and depth. The breath measure would be how many different platforms or regions are affected. Both of these pieces of information could be available through tweets. The depth measure would be how many users are affected. This measure is much simpler, it would be relative to the size of the outage spike.

Malicious Tweet Attack. SPOONS is currently susceptible to a type of attack where a user generates many fake tweets. SPOONS currently makes no attempt to verify the validity of a tweet, or the credibility of its author. Therefore, an attacker can just generate hundreds of simple tweets like: 


\section{Netflix is down.}

This will be probably be enough to cause SPOONS to report an outage when there is none.

Nature of an Outage. Currently, SPOONS cannot detect the root cause of an outage. Even though during an outage there are many tweets like:

Damn NetFlix via Xbox 360 DOWN! (Bbm Sad Face)

SPOONS does not yet try to determine the cause of an outage. 


\section{Chapter 16}

\section{Current and Future Work}

\subsection{WEKA Classifier Reimplementation}

The WEKA machine learning package offers a wide variety of classifiers. However, their implementation and API has some room for improvement. Because of this, SPOONS already uses two classifiers implemented from scratch. I plan on continuing this to make a classification package centered around performance and ease of use.

\subsection{Advanced Sentiment Analysis}

Kim Paterson, a member of the SPOONS team, is currently working on improving the sentiment analysis work from Cailin Cushing [6]. If completed, then SPOONS can use both text classification and sentiment analysis to determine when there is an outage. Because of their orthogonal natures, having both would allow SPOONS to recognize even more outages. 


\subsection{SPOONS Scaling}

Another member of the SPOONS team, Brett Armstrong, is working to improve the scalability of SPOONS. Because of its distributed architecture (see Chapter 10), SPOONS already has the potential to scale horizontally. If there is

too much traffic/work, then another server can just be added to the cluster. However, that currently requires manual intervention. Since there are spikes when outages occur, we may not know when there is going to be a lot of traffic. To solve this problem, Brett will use SPOONS to monitor itself. The end result of an Analysis Pipeline will not be an email alert, rather it will be the creation of a new AWS instance. 


\section{Bibliography}

[1] Innodb table and index structures. http://dev.mysql.com/doc/refman/5.6/en/innodbtable-and-index.html.

[2] E. Augustine, C. Cushing, A. Dekhtyar, M. Tognetti, and K. Paterson. Outage detection via real-time social stream analysis: Leveraging the power of online complaints. In $W W W$ 2012: Proceedings of the 21st World Wide Web Conference. ACM, 2012.

[3] L. Chu. Research on chinese text categorization method oriented to imbalanced corpus. may 2012.

[4] C. Cortes and V. Vapnik. Support-vector networks. Mach. Learn., 20(3):273297, Sept. 1995.

[5] A. Culotta. Detecting influenza outbreaks by analyzing twitter messages. In KDD Workshop on Social Media Analytics, 2010.

[6] C. Cushing. Detecting netflix service outages through analysis of twitter posts. Master's thesis, California Polytechnic State University - San Luis Obispo, june 2012.

[7] R. Duda, P. Hart, and D. Stork. Pattern classification. Pattern Classification and Scene Analysis: Pattern Classification. Wiley, 2001. 
[8] E. Frank and R. R. Bouckaert. Naive bayes for text classification with unbalanced classes. In Proceedings of the 10th European conference on Principle and Practice of Knowledge Discovery in Databases, PKDD'06, pages 503510, Berlin, Heidelberg, 2006. Springer-Verlag.

[9] M. Hall, E. Frank, G. Holmes, B. Pfahringer, P. Reutemann, and I. H. Witten. The WEKA data mining software: an update. SIGKDD Explor. Newsl., 11(1):10-18, 2009.

[10] L. Hong and B. D. Davison. Empirical study of topic modeling in twitter. In Proceedings of the First Workshop on Social Media Analytics, SOMA '10, pages 80-88, New York, NY, USA, 2010. ACM.

[11] F. Jabr. Using twitter to follow trends beats the stock market. NewScientist, (2829), Sept. 2011. http://www.newscientist.com/article/mg21128295.900using-twitter-to-follow-trends-beats-the-stock-market.html.

[12] L. Jiang, M. Yu, M. Zhou, X. Liu, and T. Zhao. Target-dependent twitter sentiment classification. In Proceedings of the 49th Annual Meeting of the Association for Computational Linguistics: Human Language Technologies Volume 1, HLT '11, pages 151-160, Stroudsburg, PA, USA, 2011. Association for Computational Linguistics.

[13] A. M. Kibriya, E. Frank, B. Pfahringer, and G. Holmes. Multinomial naive bayes for text categorization revisited. In Proceedings of the 17th Australian joint conference on Advances in Artificial Intelligence, AI'04, pages 488-499, Berlin, Heidelberg, 2004. Springer-Verlag.

[14] D. E. Knuth. The art of computer programming, volume 2 (3rd ed.): seminu- 
merical algorithms. Addison-Wesley Longman Publishing Co., Inc., Boston, MA, USA, 1997.

[15] K. Levchenko, B. Meeder, M. Motoyama, S. Savage, and G. M. Voelker. Measuring online service availability using twitter. In Proc. of the 3rd Workshop on Online Social Networks (WOSN 2010), 2010.

[16] Y. Matsu, M. Okazak, and T. Sakak. Earthquake shakes twitter users: real-time event detection by social sensors. In $W W W$ 2010: Proceedings of the 19th World Wide Web Conference, 2010. http://ymatsuo.com/papers/www2010.pdf.

[17] K. McEntee. personal communication, 2011.

[18] S. Mukherjee, A. Malu, B. A.R., and P. Bhattacharyya. Twisent: a multistage system for analyzing sentiment in twitter. In Proceedings of the 21st ACM international conference on Information and knowledge management, CIKM '12, pages 2531-2534, New York, NY, USA, 2012. ACM.

[19] R. E. Neapolitan. Probabilistic reasoning in expert systems: theory and algorithms. John Wiley \& Sons, Inc., New York, NY, USA, 1990.

[20] J. Pearl. Probabilistic reasoning in intelligent systems: networks of plausible inference. Morgan Kaufmann Publishers Inc., San Francisco, CA, USA, 1988.

[21] M. Pennacchiotti and A.-M. Popescu. Democrats, republicans and starbucks afficionados: user classification in twitter. In Proceedings of the 17th ACM SIGKDD international conference on Knowledge discovery and data mining, KDD '11, pages 430-438, New York, NY, USA, 2011. ACM. 
[22] J. C. Platt. Advances in kernel methods. chapter Fast training of support vector machines using sequential minimal optimization, pages 185-208. MIT Press, Cambridge, MA, USA, 1999.

[23] J. C. Platt. Advances in kernel methods. chapter Fast training of support vector machines using sequential minimal optimization, pages 185-208. MIT Press, Cambridge, MA, USA, 1999.

[24] M. F. Porter. An algorithm for suffix stripping. In K. Sparck Jones and P. Willett, editors, Readings in information retrieval, pages 313316. Morgan Kaufmann Publishers Inc., San Francisco, CA, USA, 1997. http://tartarus.org/martin/PorterStemmer.

[25] J. R. Quinlan. C4.5: programs for machine learning. Morgan Kaufmann Publishers Inc., San Francisco, CA, USA, 1993.

[26] Y. Raz. Automatic humor classification on twitter. In Proceedings of the 2012 Conference of the North American Chapter of the Association for Computational Linguistics: Human Language Technologies: Student Research Workshop, NAACL HLT '12, pages 66-70, Stroudsburg, PA, USA, 2012. Association for Computational Linguistics.

[27] H. Saif, Y. He, and H. Alani. Semantic sentiment analysis of twitter. In Proceedings of the 11th international conference on The Semantic Web Volume Part I, ISWC'12, pages 508-524, Berlin, Heidelberg, 2012. SpringerVerlag.

[28] Twitter. \#numbers, Mar. 2011. http://blog.twitter.com/2011/03/numbers.html.

[29] Twitter. Terms of service, June 2011. https://twitter.com/tos. 
[30] Twitter.

Get search/tweets,

Oct.

2012.

https://dev.twitter.com/docs/api/1.1/get/search/tweets.

[31] X. Wang, F. Wei, X. Liu, M. Zhou, and M. Zhang. Topic sentiment analysis in twitter: a graph-based hashtag sentiment classification approach. In Proceedings of the 20th ACM international conference on Information and knowledge management, CIKM '11, pages 1031-1040, New York, NY, USA, 2011. ACM.

[32] D. E. Willard. New trie data structures which support very fast search operations. J. Comput. Syst. Sci., 28(3):379-394, July 1984.

[33] W. X. Zhao, J. Jiang, J. Weng, J. He, E.-P. Lim, H. Yan, and X. Li. Comparing twitter and traditional media using topic models. In Proceedings of the 33rd European conference on Advances in information retrieval, ECIR'11, pages 338-349, Berlin, Heidelberg, 2011. Springer-Verlag. 


\section{Appendix A}

\section{SPOONS Database Schema}

\section{Highlights}

\section{A.1 DATA_tweets}

CREATE TABLE DATA_tweets (

twitter_id varchar(32) COLLATE utf8_unicode_ci NOT NULL,

published int(11) NOT NULL,

content text COLLATE utf8_unicode_ci NOT NULL,

source text COLLATE utf8_unicode_ci,

lang varchar(3) COLLATE utf8_unicode_ci NOT NULL,

author varchar(50) COLLATE utf8_unicode_ci

NOT NULL DEFAULT 'Jon Doe',

frame_id int (11) DEFAULT NULL,

id int (11) NOT NULL AUTOINCREMENT,

place text COLLATE utf8_unicode_ci, 


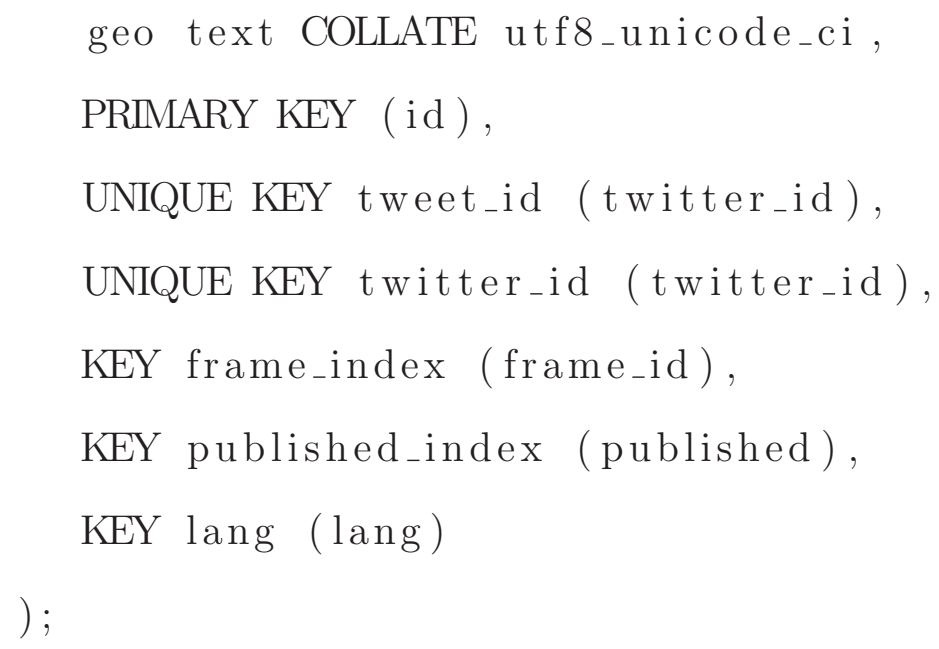




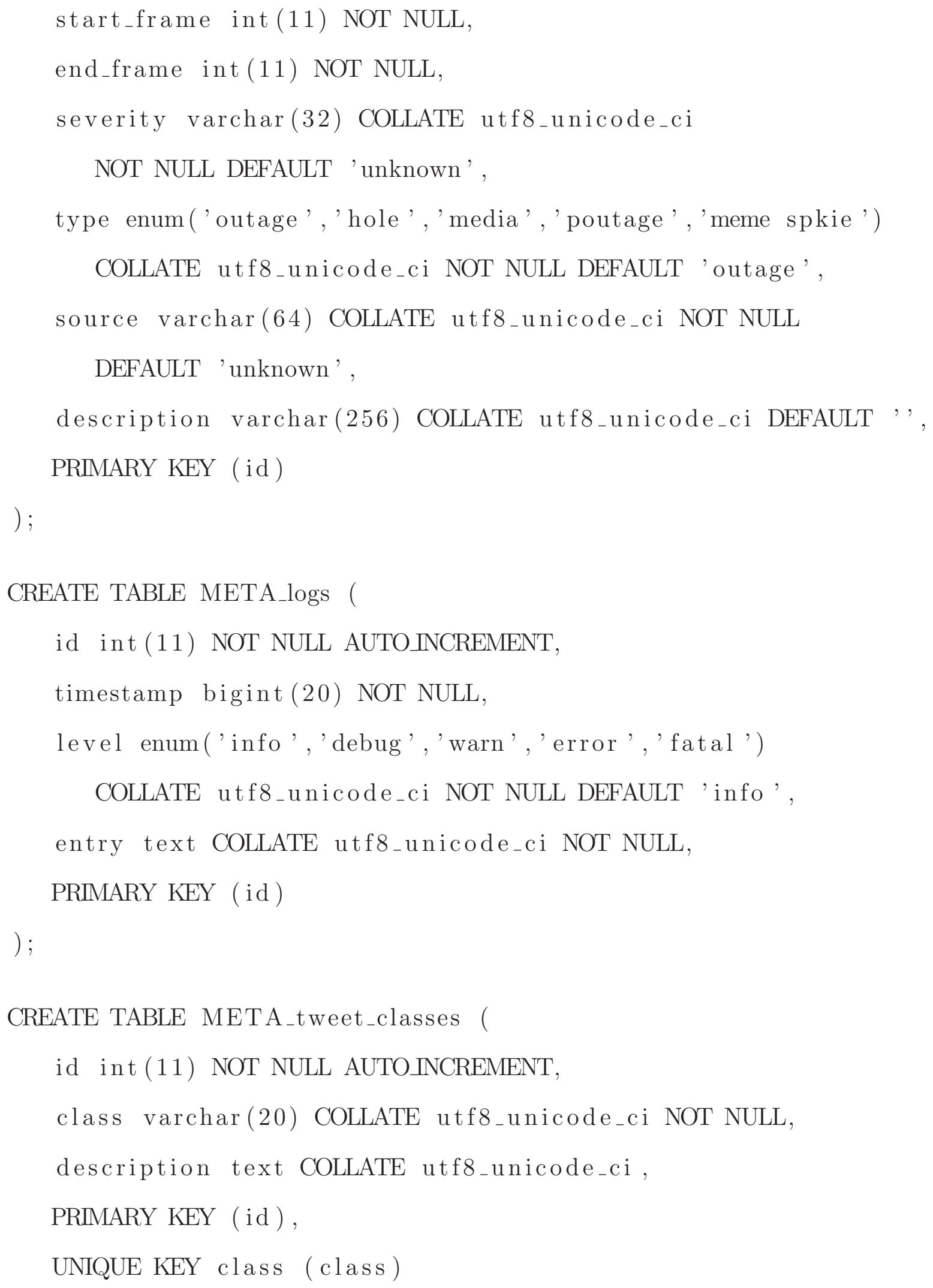




$$
)
$$

CREATE TABLE RESULT_ALL_volume (

start_frame int(11) NOT NULL,

end_frame int(11) NOT NULL,

value float DEFAULT NULL,

PRIMARY KEY ( start_frame),

UNIQUE KEY end_frame (end_frame),

KEY start_frame (start_frame),

KEY end_frame_2 (end_frame)

)

CREATE TABLE RESULT_EN_class_smo (

tweet_id int (11) NOT NULL DEFAULT '0',

class int(11) NOT NULL,

PRIMARY KEY ( tweet_id, class),

KEY tweet_id (tweet_id),

KEY class (class)

) ;

CREATE TABLE RESULT_EN_volume_smo (

start_frame int(11) NOT NULL,

end_frame int(11) NOT NULL,

undecided float NOT NULL DEFAULT '0',

media float NOT NULL DEFAULT '0',

neutral float NOT NULL DEFAULT '0',

snafu float NOT NULL DEFAULT '0',

watching float NOT NULL DEFAULT '0', 
response float NOT NULL DEFAULT '0', complaint float NOT NULL DEFAULT '0', refuse_to_rate float NOT NULL DEFAULT '0', happy float NOT NULL DEFAULT '0', PRIMARY KEY (start_frame), UNIQUE KEY end_frame (end_frame), KEY start_frame (start_frame), KEY end_frame_2 (end_frame) ) ;

CREATE TABLE RESULT_EN_volume_weighted_series_prediction_week ( start_frame int(11) NOT NULL, prediction float DEFAULT NULL, PRIMARY KEY (start_frame) ); 
Appendix B

\section{Full Classifier Evaluation Results}




\begin{tabular}{|c|c|c|c|c|c|c|c|c|c|}
\hline Classified & undecided & media & neutral & snafu & watching & response & complaint & refuse to rate & happy \\
\hline undecided & $\mathbf{0}$ & 0 & 0 & 0 & 0 & 0 & 0 & 0 & 0 \\
\hline media & 0 & $\mathbf{4 8}$ & 1 & 0 & 2 & 2 & 0 & 2 & 2 \\
\hline neutral & 0 & 50 & $\mathbf{4 0}$ & 48 & 65 & 11 & 41 & 24 & 40 \\
\hline snafu & 0 & 0 & 3 & $\mathbf{6 9}$ & 3 & 4 & 53 & 0 & 36 \\
\hline watching & 0 & 1 & 8 & 3 & $\mathbf{4 6}$ & 6 & 4 & 2 & 22 \\
\hline response & 0 & 2 & 8 & 6 & 6 & 1 & 1 & 2 & 8 \\
\hline complaint & 0 & 2 & 2 & 32 & 3 & 0 & $\mathbf{4 4}$ & 0 & 7 \\
\hline refuse to rate & 0 & 0 & 0 & 0 & 1 & 1 & 0 & $\mathbf{1 8}$ & 2 \\
\hline happy & 0 & 0 & 4 & 0 & 9 & 5 & 3 & 0 & $\mathbf{3 0}$ \\
\hline
\end{tabular}

(a) Non-Weka NaiveBayesClassifier

\begin{tabular}{|c|c|c|c|c|c|c|c|c|c|}
\hline Actual & undecided & media & neutral & snafu & watching & response & complaint & refuse to rate & happy \\
\hline undecided & $\mathbf{0}$ & 0 & 0 & 0 & 0 & 0 & 0 & 0 & 0 \\
\hline media & 0 & $\mathbf{9 3}$ & 9 & 0 & 4 & 3 & 6 & 9 & 4 \\
\hline neutral & 0 & 1 & $\mathbf{1 2}$ & 2 & 23 & 7 & 3 & 2 & 17 \\
\hline snafu & 0 & 1 & 5 & $\mathbf{1 0 4}$ & 5 & 4 & 66 & 0 & 41 \\
\hline watching & 0 & 1 & 16 & 10 & $\mathbf{8 3}$ & 9 & 6 & 2 & 32 \\
\hline response & 0 & 3 & 13 & 5 & 7 & $\mathbf{0}$ & 3 & 2 & 6 \\
\hline complaint & 0 & 3 & 4 & 34 & 2 & 0 & $\mathbf{5 7}$ & 1 & 10 \\
\hline refuse to rate & 0 & 1 & 0 & 1 & 1 & 1 & 0 & $\mathbf{2 9}$ & 2 \\
\hline happy & 0 & 0 & 7 & 2 & 10 & 6 & 5 & 3 & $\mathbf{3 5}$ \\
\hline
\end{tabular}

(b) Non-Weka BPNBClassifier

\begin{tabular}{|c|c|c|c|c|c|c|c|c|c|}
\hline Actual & undecided & media & neutral & snafu & watching & response & complaint & refuse to rate & happy \\
\hline undecided & $\mathbf{0}$ & 0 & 0 & 0 & 0 & 0 & 0 & 0 & 0 \\
\hline media & 0 & $\mathbf{8 5}$ & 0 & 0 & 0 & 2 & 1 & 6 & 1 \\
\hline neutral & 0 & 4 & $\mathbf{2 5}$ & 6 & 26 & 14 & 12 & 4 & 22 \\
\hline snafu & 0 & 1 & 3 & $\mathbf{9 4}$ & 2 & 3 & 42 & 0 & 15 \\
\hline watching & 0 & 2 & 11 & 7 & $\mathbf{8 2}$ & 6 & 8 & 1 & 14 \\
\hline response & 0 & 4 & 13 & 7 & 9 & $\mathbf{3}$ & 10 & 2 & 6 \\
\hline complaint & 0 & 0 & 3 & 25 & 1 & 0 & $\mathbf{5 4}$ & 2 & 7 \\
\hline refuse to rate & 0 & 6 & 0 & 1 & 0 & 0 & 0 & $\mathbf{3 0}$ & 0 \\
\hline happy & 0 & 1 & 11 & 18 & 15 & 2 & 19 & 3 & $\mathbf{8 2}$ \\
\hline
\end{tabular}

(c) NaiveBayesClassifier

\begin{tabular}{|c|c|c|c|c|c|c|c|c|c|}
\hline Actual & undecided & media & neutral & snafu & watching & response & complaint & refuse to rate & happy \\
\hline undecided & $\mathbf{0}$ & 0 & 0 & 0 & 0 & 0 & 0 & 0 & 0 \\
\hline media & 0 & $\mathbf{8 7}$ & 1 & 0 & 0 & 2 & 2 & 9 & 1 \\
\hline neutral & 0 & 4 & $\mathbf{1}$ & 0 & 0 & 0 & 0 & 1 & 0 \\
\hline snafu & 0 & 0 & 9 & $\mathbf{7 7}$ & 6 & 4 & 36 & 2 & 26 \\
\hline watching & 0 & 2 & 20 & 9 & $\mathbf{1 0 5}$ & 9 & 10 & 0 & 21 \\
\hline response & 0 & 0 & 0 & 0 & 1 & $\mathbf{0}$ & 0 & 0 & 0 \\
\hline complaint & 0 & 1 & 1 & 21 & 1 & 1 & $\mathbf{2 4}$ & 1 & 2 \\
\hline refuse to rate & 0 & 8 & 2 & 0 & 0 & 0 & 0 & $\mathbf{2 3}$ & 0 \\
\hline happy & 0 & 1 & 32 & 51 & 22 & 14 & 74 & 12 & $\mathbf{9 7}$ \\
\hline
\end{tabular}

(d) BayesNetClassifier

Table B.1: Uncompressed, None Filter Classification Confusion Matrices 


\begin{tabular}{|c|c|c|c|c|c|c|c|c|c|}
\hline Classified & undecided & media & neutral & snafu & watching & response & complaint & refuse to rate & happy \\
\hline undecided & $\mathbf{0}$ & 0 & 0 & 0 & 0 & 0 & 0 & 0 & 0 \\
\hline media & 0 & $\mathbf{9 5}$ & 4 & 0 & 2 & 2 & 2 & 11 & 1 \\
\hline neutral & 0 & 1 & $\mathbf{9}$ & 5 & 6 & 6 & 4 & 2 & 5 \\
\hline snafu & 0 & 0 & 5 & $\mathbf{8 4}$ & 2 & 5 & 37 & 4 & 9 \\
\hline watching & 0 & 2 & 13 & 3 & $\mathbf{8 4}$ & 5 & 5 & 0 & 17 \\
\hline response & 0 & 0 & 1 & 0 & 5 & $\mathbf{0}$ & 1 & 1 & 5 \\
\hline complaint & 0 & 1 & 7 & 36 & 7 & 1 & $\mathbf{5 4}$ & 6 & 9 \\
\hline refuse to rate & 0 & 3 & 3 & 2 & 0 & 0 & 4 & $\mathbf{1 4}$ & 0 \\
\hline happy & 0 & 1 & 24 & 28 & 29 & 11 & 39 & 10 & $\mathbf{1 0 1}$ \\
\hline
\end{tabular}

(e) J48Classifier

\begin{tabular}{|c|c|c|c|c|c|c|c|c|c|}
\hline Classified & undecided & media & neutral & snafu & watching & response & complaint & refuse to rate & happy \\
\hline undecided & $\mathbf{0}$ & 0 & 0 & 0 & 0 & 0 & 0 & 0 & 0 \\
\hline media & 0 & $\mathbf{5 6}$ & 0 & 0 & 1 & 2 & 0 & 2 & 0 \\
\hline neutral & 0 & 0 & $\mathbf{3}$ & 0 & 10 & 4 & 4 & 0 & 3 \\
\hline snafu & 0 & 0 & 2 & $\mathbf{4 5}$ & 0 & 4 & 36 & 0 & 1 \\
\hline watching & 0 & 1 & 7 & 0 & $\mathbf{7 3}$ & 5 & 2 & 1 & 10 \\
\hline response & 0 & 2 & 4 & 4 & 4 & $\mathbf{0}$ & 0 & 1 & 5 \\
\hline complaint & 0 & 0 & 5 & 59 & 5 & 1 & $\mathbf{6 1}$ & 0 & 5 \\
\hline refuse to rate & 0 & 0 & 0 & 0 & 0 & 1 & 0 & 4 & 0 \\
\hline happy & 0 & 44 & 45 & 50 & 42 & 13 & 43 & 40 & $\mathbf{1 2 3}$ \\
\hline
\end{tabular}

(f) KNNClassifier

\begin{tabular}{|c|c|c|c|c|c|c|c|c|c|}
\hline Actual & undecided & media & neutral & snafu & watching & response & complaint & refuse to rate & happy \\
\hline undecided & $\mathbf{0}$ & 0 & 0 & 0 & 0 & 0 & 0 & 0 & 0 \\
\hline media & 0 & $\mathbf{9 8}$ & 3 & 0 & 2 & 2 & 2 & 9 & 1 \\
\hline neutral & 0 & 0 & $\mathbf{1 1}$ & 1 & 12 & 5 & 5 & 1 & 9 \\
\hline snafu & 0 & 0 & 2 & $\mathbf{8 7}$ & 3 & 4 & 40 & 1 & 3 \\
\hline watching & 0 & 2 & 16 & 4 & $\mathbf{8 8}$ & 7 & 4 & 1 & 13 \\
\hline response & 0 & 2 & 7 & 4 & 5 & $\mathbf{0}$ & 1 & 1 & 6 \\
\hline complaint & 0 & 0 & 7 & 41 & 4 & 3 & $\mathbf{6 8}$ & 0 & 8 \\
\hline refuse to rate & 0 & 0 & 0 & 0 & 0 & 1 & 1 & $\mathbf{1 8}$ & 0 \\
\hline happy & 0 & 1 & 20 & 21 & 21 & 8 & 25 & 17 & $\mathbf{1 0 7}$ \\
\hline
\end{tabular}

(g) SMOClassifier

\begin{tabular}{|c|c|c|c|c|c|c|c|c|c|}
\hline Actual & undecided & media & neutral & snafu & watching & response & complaint & refuse to rate & happy \\
\hline undecided & $\mathbf{0}$ & 0 & 0 & 0 & 0 & 0 & 0 & 0 & 0 \\
\hline media & 0 & $\mathbf{9 8}$ & 1 & 0 & 0 & 2 & 1 & 13 & 1 \\
\hline neutral & 0 & 1 & $\mathbf{2 3}$ & 6 & 21 & 14 & 11 & 4 & 17 \\
\hline snafu & 0 & 0 & 2 & $\mathbf{9 7}$ & 2 & 3 & 45 & 1 & 17 \\
\hline watching & 0 & 2 & 12 & 7 & $\mathbf{9 2}$ & 6 & 10 & 0 & 19 \\
\hline response & 0 & 2 & 13 & 7 & 5 & $\mathbf{3}$ & 8 & 3 & 6 \\
\hline complaint & 0 & 0 & 4 & 25 & 2 & 0 & $\mathbf{5 3}$ & 1 & 9 \\
\hline refuse to rate & 0 & 0 & 0 & 1 & 0 & 0 & 0 & $\mathbf{2 3}$ & 0 \\
\hline happy & 0 & 0 & 11 & 15 & 13 & 2 & 18 & 3 & $\mathbf{7 8}$ \\
\hline
\end{tabular}

(h) BinaryNaiveBayesClassifier

Table B.0: Uncompressed, None Filter Classification Confusion Matrices Cont. 


\begin{tabular}{|c|c|c|c|c|c|c|c|c|c|}
\hline Classified & undecided & media & neutral & snafu & watching & response & complaint & refuse to rate & happy \\
\hline undecided & $\mathbf{0}$ & 0 & 0 & 0 & 0 & 0 & 0 & 0 & 0 \\
\hline media & 0 & $\mathbf{9 7}$ & 2 & 0 & 1 & 2 & 2 & 14 & 1 \\
\hline neutral & 0 & 0 & $\mathbf{4}$ & 0 & 4 & 2 & 1 & 0 & 3 \\
\hline snafu & 0 & 2 & 10 & $\mathbf{6 1}$ & 6 & 4 & 30 & 2 & 20 \\
\hline watching & 0 & 0 & 10 & 6 & $\mathbf{8 0}$ & 4 & 5 & 1 & 11 \\
\hline response & 0 & 0 & 0 & 0 & 0 & $\mathbf{0}$ & 0 & 0 & 0 \\
\hline complaint & 0 & 1 & 3 & 26 & 13 & 1 & $\mathbf{4 1}$ & 5 & 24 \\
\hline refuse to rate & 0 & 2 & 1 & 0 & 0 & 0 & 0 & $\mathbf{5}$ & 0 \\
\hline happy & 0 & 1 & 36 & 65 & 31 & 17 & 67 & 21 & $\mathbf{8 8}$ \\
\hline
\end{tabular}

(i) BinaryJ48Classifier

\begin{tabular}{|c|c|c|c|c|c|c|c|c|c|}
\hline Actual & undecided & media & neutral & snafu & watching & response & complaint & refuse to rate & happy \\
\hline undecided & $\mathbf{0}$ & 0 & 0 & 0 & 0 & 0 & 0 & 0 & 0 \\
\hline media & 0 & $\mathbf{9 0}$ & 2 & 0 & 0 & 2 & 1 & 10 & 1 \\
\hline neutral & 0 & 10 & $\mathbf{2}$ & 1 & 1 & 1 & 2 & 2 & 2 \\
\hline snafu & 0 & 0 & 9 & $\mathbf{8 5}$ & 4 & 4 & 33 & 2 & 26 \\
\hline watching & 0 & 2 & 18 & 6 & $\mathbf{1 0 7}$ & 9 & 10 & 0 & 19 \\
\hline response & 0 & 0 & 0 & 0 & 0 & $\mathbf{0}$ & 0 & 0 & 0 \\
\hline complaint & 0 & 0 & 32 & 64 & 20 & 14 & $\mathbf{9 9}$ & 12 & 91 \\
\hline refuse to rate & 0 & 1 & 0 & 2 & 0 & 0 & 0 & $\mathbf{2 2}$ & 0 \\
\hline happy & 0 & 0 & 3 & 0 & 3 & 0 & 1 & 0 & $\mathbf{8}$ \\
\hline
\end{tabular}

(j) BinaryBayesNetClassifier

\begin{tabular}{|c|c|c|c|c|c|c|c|c|c|}
\hline Actual & undecided & media & neutral & snafu & watching & response & complaint & refuse to rate & happy \\
\hline undecided & $\mathbf{0}$ & 0 & 0 & 0 & 0 & 0 & 0 & 0 & 0 \\
\hline media & 0 & $\mathbf{5 7}$ & 0 & 0 & 1 & 2 & 0 & 2 & 0 \\
\hline neutral & 0 & 0 & $\mathbf{3}$ & 0 & 10 & 5 & 4 & 0 & 3 \\
\hline snafu & 0 & 0 & 1 & $\mathbf{4 5}$ & 0 & 4 & 36 & 0 & 1 \\
\hline watching & 0 & 1 & 8 & 0 & $\mathbf{7 4}$ & 5 & 2 & 1 & 10 \\
\hline response & 0 & 2 & 4 & 4 & 4 & $\mathbf{0}$ & 0 & 1 & 5 \\
\hline complaint & 0 & 0 & 5 & 59 & 5 & 0 & $\mathbf{6 1}$ & 0 & 5 \\
\hline refuse to rate & 0 & 0 & 0 & 0 & 0 & 1 & 0 & 4 & 0 \\
\hline happy & 0 & 43 & 45 & 50 & 41 & 13 & 43 & 40 & $\mathbf{1 2 3}$ \\
\hline
\end{tabular}

(k) BinaryKNNClassifier

\begin{tabular}{|c|c|c|c|c|c|c|c|c|c|}
\hline Actual & undecided & media & neutral & snafu & watching & response & complaint & refuse to rate & happy \\
\hline undecided & $\mathbf{0}$ & 0 & 0 & 0 & 0 & 0 & 0 & 0 & 0 \\
\hline media & 0 & $\mathbf{9 2}$ & 1 & 0 & 2 & 2 & 1 & 9 & 1 \\
\hline neutral & 0 & 7 & $\mathbf{3 3}$ & 32 & 28 & 11 & 38 & 13 & 50 \\
\hline snafu & 0 & 0 & 3 & $\mathbf{6 9}$ & 2 & 4 & 37 & 1 & 1 \\
\hline watching & 0 & 1 & 10 & 2 & $\mathbf{8 4}$ & 5 & 3 & 0 & 13 \\
\hline response & 0 & 2 & 4 & 4 & 4 & $\mathbf{0}$ & 0 & 1 & 5 \\
\hline complaint & 0 & 1 & 3 & 43 & 2 & 0 & $\mathbf{5 5}$ & 1 & 2 \\
\hline refuse to rate & 0 & 0 & 0 & 0 & 0 & 1 & 0 & $\mathbf{1 9}$ & 0 \\
\hline happy & 0 & 0 & 12 & 8 & 13 & 7 & 12 & 4 & $\mathbf{7 5}$ \\
\hline
\end{tabular}

(l) BinarySMOClassifier

Table B.-1: Uncompressed, None Filter Classification Confusion Matrices Cont. 


\begin{tabular}{|c|c|c|c|c|c|c|c|c|c|}
\hline Classified & undecided & media & neutral & snafu & watching & response & complaint & refuse to rate & happy \\
\hline undecided & $\mathbf{0}$ & 0 & 0 & 0 & 0 & 0 & 0 & 0 & 0 \\
\hline media & 0 & $\mathbf{5 9}$ & 1 & 0 & 2 & 2 & 1 & 2 & 2 \\
\hline neutral & 0 & 36 & $\mathbf{3 9}$ & 42 & 64 & 11 & 39 & 17 & 39 \\
\hline snafu & 0 & 0 & 4 & $\mathbf{6 9}$ & 5 & 4 & 51 & 4 & 13 \\
\hline watching & 0 & 2 & 9 & 5 & $\mathbf{4 5}$ & 5 & 4 & 2 & 21 \\
\hline response & 0 & 3 & 6 & 5 & 5 & 1 & 1 & 2 & 5 \\
\hline complaint & 0 & 3 & 3 & 36 & 4 & 1 & $\mathbf{4 7}$ & 0 & 7 \\
\hline refuse to rate & 0 & 0 & 0 & 0 & 0 & 1 & 0 & $\mathbf{2 1}$ & 1 \\
\hline happy & 0 & 0 & 4 & 1 & 10 & 5 & 3 & 0 & $\mathbf{5 9}$ \\
\hline
\end{tabular}

(a) Non-Weka NaiveBayesClassifier

\begin{tabular}{|c|c|c|c|c|c|c|c|c|c|}
\hline Actual & undecided & media & neutral & snafu & watching & response & complaint & refuse to rate & happy \\
\hline undecided & $\mathbf{0}$ & 0 & 0 & 0 & 0 & 0 & 0 & 0 & 0 \\
\hline media & 0 & $\mathbf{8 8}$ & 9 & 1 & 3 & 3 & 7 & 7 & 4 \\
\hline neutral & 0 & 2 & $\mathbf{9}$ & 2 & 19 & 5 & 4 & 1 & 11 \\
\hline snafu & 0 & 4 & 5 & $\mathbf{1 0 0}$ & 8 & 4 & 66 & 4 & 18 \\
\hline watching & 0 & 2 & 19 & 9 & $\mathbf{8 2}$ & 9 & 5 & 5 & 27 \\
\hline response & 0 & 3 & 7 & 5 & 5 & $\mathbf{1}$ & 2 & 2 & 5 \\
\hline complaint & 0 & 3 & 8 & 39 & 2 & 1 & $\mathbf{5 7}$ & 1 & 10 \\
\hline refuse to rate & 0 & 1 & 0 & 0 & 1 & 1 & 0 & $\mathbf{2 6}$ & 1 \\
\hline happy & 0 & 0 & 9 & 2 & 15 & 6 & 5 & 2 & $\mathbf{7 1}$ \\
\hline
\end{tabular}

(b) Non-Weka BPNBClassifier

\begin{tabular}{|c|c|c|c|c|c|c|c|c|c|}
\hline Actual & undecided & media & neutral & snafu & watching & response & complaint & refuse to rate & happy \\
\hline undecided & $\mathbf{0}$ & 0 & 0 & 0 & 0 & 0 & 0 & 0 & 0 \\
\hline media & 0 & $\mathbf{8 1}$ & 0 & 0 & 0 & 2 & 1 & 5 & 0 \\
\hline neutral & 0 & 3 & $\mathbf{2 3}$ & 7 & 26 & 12 & 9 & 4 & 18 \\
\hline snafu & 0 & 0 & 3 & $\mathbf{8 1}$ & 0 & 3 & 36 & 1 & 5 \\
\hline watching & 0 & 1 & 14 & 8 & $\mathbf{8 2}$ & 6 & 9 & 2 & 22 \\
\hline response & 0 & 3 & 11 & 4 & 5 & $\mathbf{3}$ & 8 & 4 & 5 \\
\hline complaint & 0 & 0 & 11 & 55 & 12 & 1 & $\mathbf{7 7}$ & 9 & 31 \\
\hline refuse to rate & 0 & 14 & 1 & 2 & 0 & 0 & 3 & $\mathbf{2 1}$ & 1 \\
\hline happy & 0 & 1 & 3 & 1 & 10 & 3 & 3 & 2 & $\mathbf{6 5}$ \\
\hline
\end{tabular}

(c) NaiveBayesClassifier

\begin{tabular}{|c|c|c|c|c|c|c|c|c|c|}
\hline Actual & undecided & media & neutral & snafu & watching & response & complaint & refuse to rate & happy \\
\hline undecided & $\mathbf{0}$ & 0 & 0 & 0 & 0 & 0 & 0 & 0 & 0 \\
\hline media & 0 & $\mathbf{9 4}$ & 3 & 0 & 0 & 2 & 2 & 14 & 1 \\
\hline neutral & 0 & 1 & $\mathbf{0}$ & 0 & 3 & 0 & 0 & 0 & 0 \\
\hline snafu & 0 & 0 & 8 & $\mathbf{4 8}$ & 5 & 8 & 20 & 5 & 11 \\
\hline watching & 0 & 0 & 3 & 1 & $\mathbf{7 9}$ & 4 & 2 & 0 & 10 \\
\hline response & 0 & 0 & 1 & 2 & 2 & $\mathbf{2}$ & 4 & 0 & 2 \\
\hline complaint & 0 & 1 & 49 & 103 & 39 & 9 & $\mathbf{1 1 2}$ & 20 & 79 \\
\hline refuse to rate & 0 & 5 & 0 & 0 & 0 & 1 & 0 & $\mathbf{8}$ & 0 \\
\hline happy & 0 & 2 & 2 & 4 & 7 & 4 & 6 & 1 & $\mathbf{4 4}$ \\
\hline
\end{tabular}

(d) BayesNetClassifier

Table B.0: Uncompressed, Full Filter Classification Confusion Matrices 


\begin{tabular}{|c|c|c|c|c|c|c|c|c|c|}
\hline Classified & undecided & media & neutral & snafu & watching & response & complaint & refuse to rate & happy \\
\hline undecided & $\mathbf{0}$ & 0 & 0 & 0 & 0 & 0 & 0 & 0 & 0 \\
\hline media & 0 & $\mathbf{9 9}$ & 4 & 0 & 1 & 2 & 2 & 13 & 1 \\
\hline neutral & 0 & 0 & $\mathbf{1 0}$ & 5 & 7 & 5 & 7 & 2 & 11 \\
\hline snafu & 0 & 0 & 2 & $\mathbf{7 1}$ & 2 & 2 & 35 & 1 & 4 \\
\hline watching & 0 & 0 & 8 & 4 & $\mathbf{9 0}$ & 8 & 4 & 3 & 14 \\
\hline response & 0 & 0 & 3 & 3 & 2 & $\mathbf{0}$ & 1 & 1 & 3 \\
\hline complaint & 0 & 2 & 13 & 50 & 11 & 2 & $\mathbf{7 2}$ & 6 & 15 \\
\hline refuse to rate & 0 & 1 & 1 & 0 & 1 & 0 & 0 & $\mathbf{1 0}$ & 0 \\
\hline happy & 0 & 1 & 25 & 25 & 21 & 11 & 25 & 12 & $\mathbf{9 9}$ \\
\hline
\end{tabular}

(e) J48Classifier

\begin{tabular}{|c|c|c|c|c|c|c|c|c|c|}
\hline Classified & undecided & media & neutral & snafu & watching & response & complaint & refuse to rate & happy \\
\hline undecided & $\mathbf{0}$ & 0 & 0 & 0 & 0 & 0 & 0 & 0 & 0 \\
\hline media & 0 & $\mathbf{5 8}$ & 0 & 0 & 1 & 2 & 0 & 0 & 0 \\
\hline neutral & 0 & 17 & $\mathbf{2 1}$ & 20 & 39 & 5 & 18 & 13 & 36 \\
\hline snafu & 0 & 9 & 13 & $\mathbf{5 7}$ & 12 & 5 & 53 & 9 & 16 \\
\hline watching & 0 & 1 & 8 & 0 & $\mathbf{4 0}$ & 4 & 2 & 2 & 6 \\
\hline response & 0 & 2 & 4 & 4 & 4 & $\mathbf{0}$ & 0 & 1 & 5 \\
\hline complaint & 0 & 7 & 10 & 72 & 11 & 2 & $\mathbf{6 6}$ & 7 & 9 \\
\hline refuse to rate & 0 & 0 & 0 & 0 & 0 & 1 & 0 & $\mathbf{4}$ & 0 \\
\hline happy & 0 & 9 & 10 & 5 & 28 & 11 & 7 & 12 & $\mathbf{7 5}$ \\
\hline
\end{tabular}

(f) KNNClassifier

\begin{tabular}{|c|c|c|c|c|c|c|c|c|c|}
\hline Actual & undecided & media & neutral & snafu & watching & response & complaint & refuse to rate & happy \\
\hline undecided & $\mathbf{0}$ & 0 & 0 & 0 & 0 & 0 & 0 & 0 & 0 \\
\hline media & 0 & $\mathbf{9 8}$ & 4 & 0 & 2 & 2 & 2 & 11 & 1 \\
\hline neutral & 0 & 1 & $\mathbf{7}$ & 3 & 13 & 6 & 4 & 0 & 7 \\
\hline snafu & 0 & 0 & 3 & $\mathbf{8 6}$ & 2 & 4 & 48 & 1 & 6 \\
\hline watching & 0 & 1 & 15 & 2 & $\mathbf{9 2}$ & 5 & 5 & 2 & 16 \\
\hline response & 0 & 2 & 5 & 4 & 5 & $\mathbf{1}$ & 3 & 2 & 5 \\
\hline complaint & 0 & 0 & 6 & 50 & 8 & 1 & $\mathbf{7 4}$ & 6 & 10 \\
\hline refuse to rate & 0 & 1 & 0 & 0 & 0 & 1 & 0 & $\mathbf{8}$ & 0 \\
\hline happy & 0 & 0 & 26 & 13 & 13 & 10 & 10 & 18 & $\mathbf{1 0 2}$ \\
\hline
\end{tabular}

(g) SMOClassifier

\begin{tabular}{|c|c|c|c|c|c|c|c|c|c|}
\hline Actual & undecided & media & neutral & snafu & watching & response & complaint & refuse to rate & happy \\
\hline undecided & $\mathbf{0}$ & 0 & 0 & 0 & 0 & 0 & 0 & 0 & 0 \\
\hline media & 0 & $\mathbf{9 5}$ & 2 & 0 & 0 & 2 & 1 & 9 & 0 \\
\hline neutral & 0 & 2 & $\mathbf{2 3}$ & 7 & 21 & 12 & 10 & 4 & 16 \\
\hline snafu & 0 & 0 & 4 & $\mathbf{8 5}$ & 0 & 3 & 42 & 1 & 6 \\
\hline watching & 0 & 1 & 14 & 8 & $\mathbf{8 7}$ & 6 & 9 & 2 & 21 \\
\hline response & 0 & 2 & 9 & 4 & 5 & $\mathbf{3}$ & 7 & 4 & 5 \\
\hline complaint & 0 & 0 & 11 & 52 & 12 & 1 & $\mathbf{7 3}$ & 9 & 30 \\
\hline refuse to rate & 0 & 2 & 0 & 1 & 0 & 0 & 2 & $\mathbf{1 7}$ & 1 \\
\hline happy & 0 & 1 & 3 & 1 & 10 & 3 & 2 & 2 & $\mathbf{6 8}$ \\
\hline
\end{tabular}

(h) BinaryNaiveBayesClassifier

Table B.-1: Uncompressed, Full Filter Classification Confusion Matrices Cont. 


\begin{tabular}{|c|c|c|c|c|c|c|c|c|c|}
\hline Classified & undecided & media & neutral & snafu & watching & response & complaint & refuse to rate & happy \\
\hline undecided & $\mathbf{0}$ & 0 & 0 & 0 & 0 & 0 & 0 & 0 & 0 \\
\hline media & 0 & $\mathbf{9 9}$ & 4 & 0 & 1 & 2 & 2 & 14 & 0 \\
\hline neutral & 0 & 0 & $\mathbf{0}$ & 0 & 0 & 1 & 0 & 0 & 1 \\
\hline snafu & 0 & 2 & 25 & $\mathbf{9 7}$ & 28 & 2 & 80 & 14 & 58 \\
\hline watching & 0 & 0 & 13 & 6 & $\mathbf{7 8}$ & 5 & 2 & 3 & 10 \\
\hline response & 0 & 0 & 0 & 0 & 0 & $\mathbf{0}$ & 0 & 0 & 0 \\
\hline complaint & 0 & 0 & 22 & 55 & 23 & 18 & $\mathbf{5 9}$ & 12 & 30 \\
\hline refuse to rate & 0 & 0 & 0 & 0 & 0 & 1 & 0 & $\mathbf{4}$ & 0 \\
\hline happy & 0 & 2 & 2 & 0 & 5 & 1 & 3 & 1 & $\mathbf{4 8}$ \\
\hline
\end{tabular}

(i) BinaryJ48Classifier

\begin{tabular}{|c|c|c|c|c|c|c|c|c|c|}
\hline Actual & undecided & media & neutral & snafu & watching & response & complaint & refuse to rate & happy \\
\hline undecided & $\mathbf{0}$ & 0 & 0 & 0 & 0 & 0 & 0 & 0 & 0 \\
\hline media & 0 & $\mathbf{9 5}$ & 5 & 0 & 0 & 2 & 2 & 13 & 0 \\
\hline neutral & 0 & 0 & $\mathbf{1}$ & 1 & 1 & 1 & 1 & 0 & 2 \\
\hline snafu & 0 & 0 & 19 & $\mathbf{7 3}$ & 10 & 6 & 27 & 6 & 18 \\
\hline watching & 0 & 0 & 3 & 1 & $\mathbf{7 5}$ & 4 & 2 & 0 & 9 \\
\hline response & 0 & 4 & 0 & 0 & 0 & $\mathbf{0}$ & 0 & 2 & 0 \\
\hline complaint & 0 & 1 & 34 & 79 & 32 & 11 & $\mathbf{1 0 7}$ & 17 & 63 \\
\hline refuse to rate & 0 & 1 & 0 & 0 & 0 & 0 & 0 & $\mathbf{9}$ & 0 \\
\hline happy & 0 & 2 & 4 & 4 & 17 & 6 & 7 & 1 & $\mathbf{5 5}$ \\
\hline
\end{tabular}

(j) BinaryBayesNetClassifier

\begin{tabular}{|c|c|c|c|c|c|c|c|c|c|}
\hline Actual & undecided & media & neutral & snafu & watching & response & complaint & refuse to rate & happy \\
\hline undecided & $\mathbf{0}$ & 0 & 0 & 0 & 0 & 0 & 0 & 0 & 0 \\
\hline media & 0 & $\mathbf{5 7}$ & 0 & 0 & 1 & 2 & 0 & 0 & 0 \\
\hline neutral & 0 & 19 & $\mathbf{2 3}$ & 22 & 41 & 7 & 18 & 16 & 40 \\
\hline snafu & 0 & 11 & 14 & $\mathbf{5 7}$ & 12 & 7 & 57 & 13 & 17 \\
\hline watching & 0 & 1 & 8 & 0 & $\mathbf{4 2}$ & 4 & 3 & 2 & 6 \\
\hline response & 0 & 2 & 4 & 4 & 4 & $\mathbf{0}$ & 0 & 1 & 5 \\
\hline complaint & 0 & 7 & 10 & 72 & 11 & 1 & $\mathbf{6 6}$ & 7 & 9 \\
\hline refuse to rate & 0 & 0 & 0 & 0 & 0 & 1 & 0 & 4 & 0 \\
\hline happy & 0 & 6 & 7 & 3 & 24 & 8 & 2 & 5 & 70 \\
\hline
\end{tabular}

(k) BinaryKNNClassifier

\begin{tabular}{|c|c|c|c|c|c|c|c|c|c|}
\hline Actual & undecided & media & neutral & snafu & watching & response & complaint & refuse to rate & happy \\
\hline undecided & $\mathbf{0}$ & 0 & 0 & 0 & 0 & 0 & 0 & 0 & 0 \\
\hline media & 0 & $\mathbf{9 6}$ & 4 & 0 & 2 & 2 & 2 & 10 & 1 \\
\hline neutral & 0 & 4 & $\mathbf{3 7}$ & 44 & 32 & 11 & 43 & 24 & 46 \\
\hline snafu & 0 & 0 & 2 & $\mathbf{7 0}$ & 1 & 4 & 38 & 0 & 1 \\
\hline watching & 0 & 1 & 8 & 2 & $\mathbf{8 4}$ & 6 & 4 & 1 & 13 \\
\hline response & 0 & 2 & 4 & 4 & 5 & $\mathbf{0}$ & 0 & 1 & 5 \\
\hline complaint & 0 & 0 & 3 & 37 & 4 & 0 & $\mathbf{5 7}$ & 1 & 3 \\
\hline refuse to rate & 0 & 0 & 0 & 0 & 0 & 1 & 0 & $\mathbf{9}$ & 0 \\
\hline happy & 0 & 0 & 8 & 1 & 7 & 6 & 2 & 2 & $\mathbf{7 8}$ \\
\hline
\end{tabular}

(l) BinarySMOClassifier

Table B.-2: Uncompressed, Full Filter Classification Confusion Matrices Cont. 


\begin{tabular}{|c|c|c|c|}
\hline Classified & media & snafu & other \\
\hline media & 48 & 0 & 9 \\
\hline snafu & 2 & 198 & 58 \\
\hline other & 53 & 106 & 359 \\
\hline
\end{tabular}

(a) Non-Weka NaiveBayesClassifier

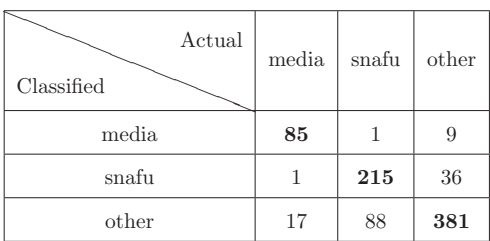

(c) NaiveBayesClassifier

\begin{tabular}{|c|c|c|c|}
\hline Classified & media & snafu & other \\
\hline media & 95 & 2 & 20 \\
\hline snafu & 1 & 211 & 55 \\
\hline other & 7 & 91 & 351 \\
\hline
\end{tabular}

(e) J48Classifier

\begin{tabular}{|c|c|c|c|}
\hline Classified & media & snafu & other \\
\hline media & 98 & 2 & 17 \\
\hline snafu & 0 & 236 & 35 \\
\hline other & 5 & 66 & 374 \\
\hline
\end{tabular}

(g) SMOClassifier

\begin{tabular}{|c|c|c|c|}
\hline Classified & media & snafu & other \\
\hline media & 97 & 2 & 20 \\
\hline snafu & 3 & 158 & 88 \\
\hline other & 3 & 144 & 318 \\
\hline
\end{tabular}

(i) BinaryJ48Classifier

\begin{tabular}{|c|c|c|c|}
\hline Classified & media & snafu & other \\
\hline media & 57 & 0 & 5 \\
\hline snafu & 0 & 201 & 21 \\
\hline other & 46 & 103 & 400 \\
\hline
\end{tabular}

(k) BinaryKNNClassifier

\begin{tabular}{|c|c|c|c|}
\hline Classified Actual & media & snafu & other \\
\hline media & $\mathbf{9 3}$ & 6 & 29 \\
\hline snafu & 4 & $\mathbf{2 6 1}$ & 72 \\
\hline other & 6 & 37 & $\mathbf{3 2 5}$ \\
\hline
\end{tabular}

(b) Non-Weka BPNBClassifier

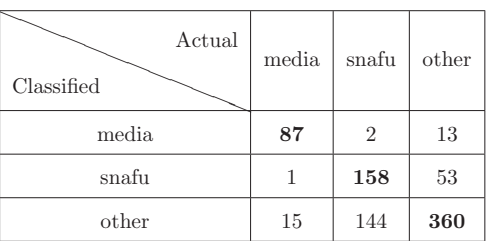

(d) BayesNetClassifier

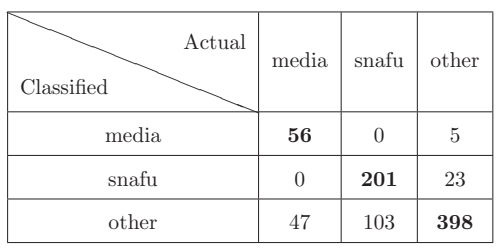

(f) KNNClassifier

\begin{tabular}{|c|c|c|c|}
\hline Classified & media & snafu & other \\
\hline media & 98 & 1 & 17 \\
\hline snafu & 0 & 220 & 41 \\
\hline other & 5 & 83 & 368 \\
\hline
\end{tabular}

(h) BinaryNaiveBayesClassifier

\begin{tabular}{|c|c|c|c|}
\hline Classified & media & snafu & other \\
\hline media & 90 & 1 & 15 \\
\hline snafu & 0 & 281 & 214 \\
\hline other & 13 & 22 & 197 \\
\hline
\end{tabular}

(j) BinaryBayesNetClassifier

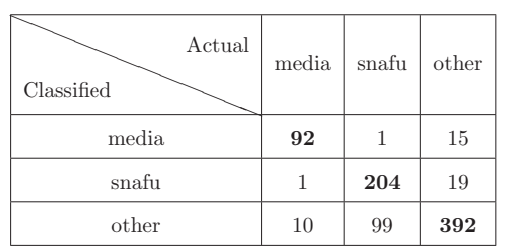

(1) BinarySMOClassifier

Table B.-1: Compressed, None Filter Classification Confusion Matrices 


\begin{tabular}{|c|c|c|c|}
\hline Classified & media & snafu & other \\
\hline media & 59 & 1 & 9 \\
\hline snafu & 3 & 203 & 45 \\
\hline other & 41 & 100 & 372 \\
\hline
\end{tabular}

(a) Non-Weka NaiveBayesClassifier

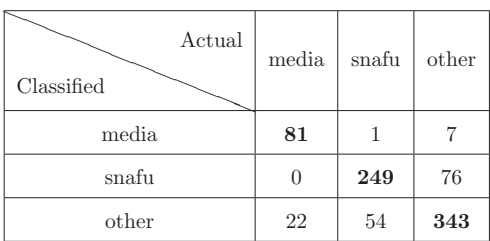

(c) NaiveBayesClassifier

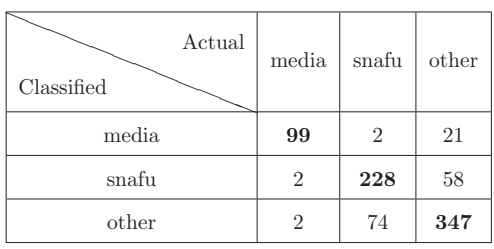

(e) J48Classifier

\begin{tabular}{|c|c|c|c|}
\hline Classified & media & snafu & other \\
\hline media & 98 & 2 & 20 \\
\hline snafu & 0 & 258 & 47 \\
\hline other & 5 & 44 & 359 \\
\hline
\end{tabular}

(g) SMOClassifier

\begin{tabular}{|c|c|c|c|}
\hline Classified & media & snafu & other \\
\hline media & 99 & 2 & 21 \\
\hline snafu & 2 & 291 & 232 \\
\hline other & 2 & 11 & 173 \\
\hline
\end{tabular}

(i) BinaryJ48Classifier

\begin{tabular}{|c|c|c|c|}
\hline Classified & media & snafu & other \\
\hline media & 57 & 0 & 3 \\
\hline snafu & 18 & 252 & 101 \\
\hline other & 28 & 52 & 322 \\
\hline
\end{tabular}

(k) BinaryKNNClassifier

\begin{tabular}{|c|c|c|c|}
\hline Classified Actual & media & snafu & other \\
\hline media & $\mathbf{8 8}$ & 8 & 26 \\
\hline snafu & 7 & $\mathbf{2 6 2}$ & 61 \\
\hline other & 8 & 34 & $\mathbf{3 3 9}$ \\
\hline
\end{tabular}

(b) Non-Weka BPNBClassifier

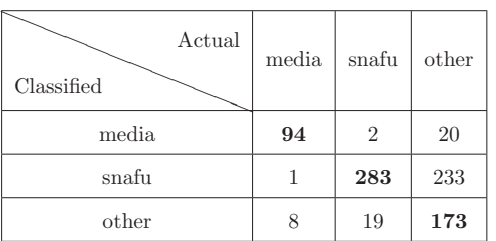

(d) BayesNetClassifier

\begin{tabular}{|c|c|c|c|}
\hline Classified & media & snafu & other \\
\hline media & 58 & 0 & 3 \\
\hline snafu & 16 & 248 & 94 \\
\hline other & 29 & 56 & 329 \\
\hline
\end{tabular}

(f) KNNClassifier

\begin{tabular}{|c|c|c|c|}
\hline Classified & media & snafu & other \\
\hline media & 95 & 1 & 13 \\
\hline snafu & 0 & 252 & 77 \\
\hline other & 8 & 51 & 336 \\
\hline
\end{tabular}

(h) BinaryNaiveBayesClassifier

\begin{tabular}{|c|c|c|c|}
\hline Classified & media & snafu & other \\
\hline media & 95 & 2 & 20 \\
\hline snafu & 1 & 286 & 216 \\
\hline other & 7 & 16 & 190 \\
\hline
\end{tabular}

(j) BinaryBayesNetClassifier

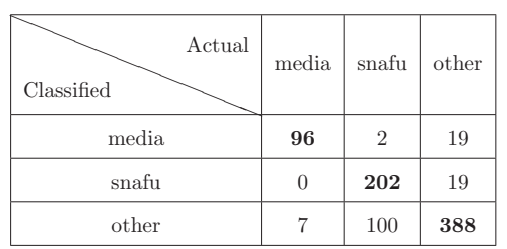

(1) BinarySMOClassifier

Table B.0: Compressed, Full Filter Classification Confusion Matrices 


\section{Appendix $\mathrm{C}$}

\section{Full Outage Detection Evaluation \\ Results}

\begin{tabular}{|c|c|c|c|c|}
\hline Parameter & Value & & & \\
\hline Resistance Method & Fighting & & & \\
\hline Alert Resistance & 10 & \multirow{2}{*}{ Predicted Actual } & \multirow{2}{*}{ True } & \multirow{2}{*}{ False } \\
\hline Recovery Resistance & 9 & & & \\
\hline Smoothing Method & None & True & 92 & 4 \\
\hline Lower Tolerance & 1.0 & False & 112 & $\mathrm{X}$ \\
\hline Upper Tolerance & 6.0 & & & \\
\hline Window Size & 10 & & & \\
\hline
\end{tabular}

Table C.1: The parameters and confusion matrix for SMO (full filtering) using the WeeklyWindowStdDev Monitor. 


\begin{tabular}{|c|c|c|c|c|}
\hline Parameter & Value & \multirow{4}{*}{ Predicted Actual } & \multirow[b]{4}{*}{ True } & \multirow[b]{4}{*}{ False } \\
\hline Resistance Method & Continuious & & & \\
\hline Alert Resistance & 9 & & & \\
\hline Recovery Resistance & 9 & & & \\
\hline Smoothing Method & Moving Mean & True & 92 & 12 \\
\hline Smoothing Window Size & 90 & False & \multirow{2}{*}{112} & \multirow{2}{*}{$\mathrm{X}$} \\
\hline Lower Tolerance & 2.0 & I dase & & \\
\hline Upper Tolerance & 4.0 & & & \\
\hline Window Size & 10 & & & \\
\hline
\end{tabular}

Table C.2: The parameters and confusion matrix for BinaryNaiveBayes (full filtering) using the WeeklyWindowStdDev Monitor.

\begin{tabular}{|c|c|c|c|c|}
\hline Parameter & Value & \multirow{4}{*}{ Predicted Actual } & \multirow{4}{*}{ True } & \multirow{4}{*}{ False } \\
\hline Resistance Method & Fighting & & & \\
\hline Alert Resistance & 10 & & & \\
\hline Recovery Resistance & 6 & & & \\
\hline Smoothing Method & None & True & 84 & 4 \\
\hline Lower Tolerance & 1.0 & False & 120 & $\mathrm{X}$ \\
\hline Upper Tolerance & 6.0 & & & \\
\hline Window Size & 10 & & & \\
\hline
\end{tabular}

Table C.3: The parameters and confusion matrix for J48 (full filtering) using the WeeklyWindowStdDev Monitor. 


\begin{tabular}{|c|c|c|c|c|}
\hline Parameter & Value & \multirow{4}{*}{ Predicted Actual } & \multirow{4}{*}{ True } & \multirow{4}{*}{ False } \\
\hline Resistance Method & \multirow{2}{*}{$\begin{array}{c}\text { Fighting } \\
10\end{array}$} & & & \\
\hline Alert Resistance & & & & \\
\hline Recovery Resistance & 7 & & & \\
\hline Smoothing Method & None & True & 87 & 3 \\
\hline Lower Tolerance & 1.0 & False & 117 & $\mathrm{X}$ \\
\hline Upper Tolerance & 5.0 & & & \\
\hline Window Size & 20 & & & \\
\hline
\end{tabular}

Table C.4: The parameters and confusion matrix for BPNB (no filtering) using the WeeklyWindowStdDev Monitor.

\begin{tabular}{|c|c|c|c|c|}
\hline Parameter & Value & \multirow{4}{*}{ Predicted Actual } & \multirow{4}{*}{ True } & \multirow{4}{*}{ False } \\
\hline Resistance Method & Fighting & & & \\
\hline Alert Resistance & 8 & & & \\
\hline Recovery Resistance & 8 & & & \\
\hline Smoothing Method & None & True & 93 & 4 \\
\hline Lower Tolerance & 1.0 & False & 111 & $\mathrm{X}$ \\
\hline Upper Tolerance & 4.0 & & & \\
\hline Window Size & 10 & & & \\
\hline
\end{tabular}

Table C.5: The parameters and confusion matrix for BinarySMO (no filtering) using the WeeklyWindowStdDev Monitor. 


\begin{tabular}{|c|c|c|c|c|}
\hline Parameter & Value & \multirow{4}{*}{ Predicted Actual } & \multirow{4}{*}{ True } & \multirow{4}{*}{ False } \\
\hline Resistance Method & \multirow{2}{*}{$\begin{array}{c}\text { Fighting } \\
10\end{array}$} & & & \\
\hline Alert Resistance & & & & \\
\hline Recovery Resistance & 10 & & & \\
\hline Smoothing Method & None & True & 85 & 3 \\
\hline Lower Tolerance & 1.0 & False & 119 & $\mathrm{X}$ \\
\hline Upper Tolerance & 5.0 & & & \\
\hline Window Size & 10 & & & \\
\hline
\end{tabular}

Table C.6: The parameters and confusion matrix for SMO (no filtering) using the WeeklyWindowStdDev Monitor.

\begin{tabular}{|c|c|c|c|c|}
\hline Parameter & Value & \multirow{4}{*}{ Predicted Actual } & \multirow{4}{*}{ True } & \multirow{4}{*}{ False } \\
\hline Resistance Method & Fighting & & & \\
\hline Alert Resistance & 10 & & & \\
\hline Recovery Resistance & 8 & & & \\
\hline Smoothing Method & None & True & 77 & 3 \\
\hline Lower Tolerance & 1.0 & False & 127 & $\mathrm{X}$ \\
\hline Upper Tolerance & 6.0 & & & \\
\hline Window Size & 20 & & & \\
\hline
\end{tabular}

Table C.7: The parameters and confusion matrix for BinaryNaiveBayes (no filtering) using the WeeklyWindowStdDev Monitor. 


\begin{tabular}{|c|c|c|c|c|}
\hline Parameter & Value & \multirow{4}{*}{ Predicted Actual } & \multirow{4}{*}{ True } & \multirow{4}{*}{ False } \\
\hline Resistance Method & \multirow{2}{*}{$\begin{array}{c}\text { Fighting } \\
10\end{array}$} & & & \\
\hline Alert Resistance & & & & \\
\hline Recovery Resistance & 5 & & & \\
\hline Smoothing Method & None & True & 81 & 7 \\
\hline Lower Tolerance & 1.0 & False & 123 & $\mathrm{X}$ \\
\hline Upper Tolerance & 9.0 & & & \\
\hline Window Size & 10 & & & \\
\hline
\end{tabular}

Table C.8: The parameters and confusion matrix for NaiveBayes (no filtering) using the WeeklyWindowStdDev Monitor.

\begin{tabular}{|c|c|c|c|c|}
\hline Parameter & Value & \multirow{4}{*}{ Predicted Actual } & \multirow[b]{4}{*}{ True } & \multirow[b]{4}{*}{ False } \\
\hline Resistance Method & Continuious & & & \\
\hline Alert Resistance & 6 & & & \\
\hline Recovery Resistance & 10 & & & \\
\hline Smoothing Method & Moving Mean & True & 67 & 12 \\
\hline Smoothing Window Size & 80 & False & 137 & $\mathrm{X}$ \\
\hline Lower Tolerance & 3.0 & & & \\
\hline Upper Tolerance & 4.0 & & & \\
\hline Window Size & 15 & & & \\
\hline
\end{tabular}

Table C.9: The parameters and confusion matrix for BinarySMO (full filtering) using the WeeklyWindowStdDev Monitor. 


\begin{tabular}{|c|c|c|c|c|}
\hline Parameter & Value & \multirow{4}{*}{ Predicted Actual } & \multirow{4}{*}{ True } & \multirow{4}{*}{ False } \\
\hline Resistance Method & \multirow{2}{*}{$\begin{array}{c}\text { Fighting } \\
10\end{array}$} & & & \\
\hline Alert Resistance & & & & \\
\hline Recovery Resistance & 5 & & & \\
\hline Smoothing Method & None & True & 101 & 8 \\
\hline Lower Tolerance & 1.0 & False & 103 & $\mathrm{X}$ \\
\hline Upper Tolerance & 6.0 & & & \\
\hline Window Size & 10 & & & \\
\hline
\end{tabular}

Table C.10: The parameters and confusion matrix for BPNB (full filtering) using the WeeklyWindowStdDev Monitor.

\begin{tabular}{|c|c|c|c|c|}
\hline Parameter & Value & \multirow{3}{*}{ Predicted Actual } & \multirow[b]{3}{*}{ True } & \multirow[b]{3}{*}{ False } \\
\hline Resistance Method & Continuious & & & \\
\hline Alert Resistance & 2 & & & \\
\hline Recovery Resistance & 8 & \multirow{2}{*}{ True } & \multirow{2}{*}{68} & \multirow{2}{*}{8} \\
\hline Smoothing Method & Moving Mean & & & \\
\hline Smoothing Window Size & 50 & \multirow{2}{*}{ False } & \multirow{2}{*}{136} & \multirow{2}{*}{$\mathrm{X}$} \\
\hline Baseline & 50.0 & & & \\
\hline
\end{tabular}

Table C.11: The parameters and confusion matrix for BinarySMO (full filtering) using the Baseline Monitor.

\begin{tabular}{|c|c|c|c|c|}
\hline Parameter & Value & & & \\
\hline Resistance Method & Continuious & \multirow{2}{*}{ Predicted Actual } & \multirow{2}{*}{ True } & \multirow{2}{*}{ False } \\
\hline Alert Resistance & 10 & & & \\
\hline Recovery Resistance & 10 & True & 84 & 5 \\
\hline Tolerance & 0.8500 & False & 120 & $\mathrm{X}$ \\
\hline Smoothing Method & None & & & \\
\hline
\end{tabular}

Table C.12: The parameters and confusion matrix for J48 (full filtering) using the Ratio Monitor. 


\begin{tabular}{|c|c|c|c|c|}
\hline Parameter & Value & \multirow{4}{*}{ Predicted Actual } & \multirow{4}{*}{ True } & \multirow{4}{*}{ False } \\
\hline Resistance Method & \multirow{2}{*}{$\begin{array}{c}\text { Continuious } \\
1 \\
\end{array}$} & & & \\
\hline Alert Resistance & & & & \\
\hline Recovery Resistance & 9 & & & \\
\hline Tolerance & 1900.0000 & True & 70 & 2 \\
\hline Smoothing Method & Moving Mean & False & 134 & $\mathrm{X}$ \\
\hline Smoothing Window Size & 90 & & & \\
\hline Window Size & 40 & & & \\
\hline
\end{tabular}

Table C.13: The parameters and confusion matrix for BinaryNaiveBayes (no filtering) using the MSE Monitor.

\begin{tabular}{|l|c|c|c|c|}
\hline Parameter & Value \\
\hline Resistance Method & Fighting & \multicolumn{3}{|c|}{} \\
\hline Alert Resistance & 8 & Actual & True & False \\
\hline Recovery Resistance & 10 & True & 83 & 13 \\
\hline Tolerance & 1800.0000 & False & 121 & X \\
\hline Smoothing Method & Moving Mean & & & \\
\hline Smoothing Window Size & 30 & & & \\
\hline Window Size & 20 & & & \\
\hline
\end{tabular}

Table C.14: The parameters and confusion matrix for NaiveBayes (no filtering) using the MSE Monitor. 


\begin{tabular}{|c|c|c|c|c|}
\hline Parameter & Value & & & \multirow[b]{3}{*}{ False } \\
\hline Resistance Method & Fighting & \multirow{3}{*}{ Predicted } & \multirow[b]{2}{*}{ True } & \\
\hline Alert Resistance & 2 & & & \\
\hline Recovery Resistance & 1 & & \multirow{2}{*}{58} & \multirow{2}{*}{3} \\
\hline Smoothing Method & Moving Mean & True & & \\
\hline Smoothing Window Size & 50 & \multirow{2}{*}{ False } & \multirow{2}{*}{146} & \multirow{2}{*}{$\mathrm{X}$} \\
\hline Baseline & 80.0 & & & \\
\hline
\end{tabular}

Table C.15: The parameters and confusion matrix for J48 (full filtering) using the Baseline Monitor.

\begin{tabular}{|c|c|c|c|c|}
\hline Parameter & Value & \multirow{4}{*}{ Predicted Actual } & \multirow[b]{3}{*}{ True } & \multirow[b]{3}{*}{ False } \\
\hline Resistance Method & Continuious & & & \\
\hline Alert Resistance & 1 & & & \\
\hline Recovery Resistance & 3 & & \multirow{2}{*}{64} & \multirow{2}{*}{9} \\
\hline Smoothing Method & Moving Mean & True & & \\
\hline Smoothing Window Size & 50 & \multirow{2}{*}{ False } & \multirow{2}{*}{140} & \multirow{2}{*}{$\mathrm{X}$} \\
\hline Baseline & 60.0 & & & \\
\hline
\end{tabular}

Table C.16: The parameters and confusion matrix for SMO (no filtering) using the Baseline Monitor.

\begin{tabular}{|c|c|c|c|c|}
\hline Parameter & Value & \multirow{3}{*}{ Predicted Actual } & \multirow[b]{3}{*}{ True } & \multirow[b]{3}{*}{ False } \\
\hline Resistance Method & Continuious & & & \\
\hline Alert Resistance & 2 & & & \\
\hline Recovery Resistance & 8 & \multirow{2}{*}{ True } & \multirow{2}{*}{68} & \multirow{2}{*}{7} \\
\hline \multirow{2}{*}{ Smoothing Method } & Moving Mean & & & \\
\hline & & \multirow[t]{3}{*}{ False } & \multirow[t]{3}{*}{136} & $\mathrm{X}$ \\
\hline smootning winaow size & 50 & & & \\
\hline Baseline & 90.0 & & & \\
\hline
\end{tabular}

Table C.17: The parameters and confusion matrix for BinaryNaiveBayes (full filtering) using the Baseline Monitor. 


\begin{tabular}{|c|c|c|c|c|}
\hline Parameter & Value & \multirow{3}{*}{ Predicted Actual } & \multirow[b]{3}{*}{ True } & \multirow[b]{3}{*}{ False } \\
\hline Resistance Method & Continuious & & & \\
\hline Alert Resistance & 1 & & & \\
\hline Recovery Resistance & 7 & True & 87 & 16 \\
\hline Smoothing Method & Moving Mean & \multirow{3}{*}{ False } & \multirow{3}{*}{117} & \multirow{3}{*}{$\mathrm{X}$} \\
\hline Smoothing Window Size & 50 & & & \\
\hline Baseline & 40.0 & & & \\
\hline
\end{tabular}

Table C.18: The parameters and confusion matrix for NaiveBayes (no filtering) using the Baseline Monitor.

\begin{tabular}{|c|c|c|c|c|}
\hline Parameter & Value & \multirow{3}{*}{ Predicted Actual } & \multirow[b]{3}{*}{ True } & \multirow[b]{3}{*}{ False } \\
\hline Resistance Method & Fighting & & & \\
\hline Alert Resistance & 1 & & & \\
\hline Recovery Resistance & 6 & \multirow{2}{*}{ True } & \multirow{2}{*}{81} & \multirow{2}{*}{9} \\
\hline Smoothing Method & Moving Mean & & & \\
\hline Smoothing Window Size & & \multirow{2}{*}{ False } & \multirow{2}{*}{123} & \multirow{2}{*}{$\mathrm{X}$} \\
\hline Baseline & 40.0 & & & \\
\hline
\end{tabular}

Table C.19: The parameters and confusion matrix for BinaryNaiveBayes (no filtering) using the Baseline Monitor.

\begin{tabular}{|c|c|c|c|c|}
\hline Parameter & Value & \multirow{3}{*}{ Predicted Actual } & \multirow[b]{3}{*}{ True } & \multirow[b]{3}{*}{ False } \\
\hline Resistance Method & Window & & & \\
\hline Window Size & 6 & & & \\
\hline Control & 2 & \multirow{2}{*}{ True } & \multirow{2}{*}{91} & \multirow{2}{*}{23} \\
\hline Tolerance & 1900.0000 & & & \\
\hline Smoothing Method & None & \multirow{2}{*}{ False } & \multirow{2}{*}{113} & \multirow{2}{*}{$\mathrm{X}$} \\
\hline Window Size & 40 & & & \\
\hline
\end{tabular}

Table C.20: The parameters and confusion matrix for BinarySMO (no filtering) using the MSE Monitor. 


\begin{tabular}{|c|c|c|c|c|}
\hline Parameter & Value & \multirow{4}{*}{ Predicted Actual } & \multirow{4}{*}{ True } & \multirow{4}{*}{ False } \\
\hline Resistance Method & Fighting & & & \\
\hline Alert Resistance & 10 & & & \\
\hline Recovery Resistance & 10 & & & \\
\hline Tolerance & 1800.0000 & True & 82 & 16 \\
\hline Smoothing Method & Moving Mean & False & 122 & $\mathrm{X}$ \\
\hline Smoothing Window Size & 10 & & & \\
\hline Window Size & 30 & & & \\
\hline
\end{tabular}

Table C.21: The parameters and confusion matrix for BinarySMO (full filtering) using the MSE Monitor.

\begin{tabular}{|c|c|c|c|c|}
\hline Parameter & Value & \multirow{3}{*}{ Predicted Actual } & \multirow[b]{3}{*}{ True } & \multirow[b]{3}{*}{ False } \\
\hline Resistance Method & Continuious & & & \\
\hline Alert Resistance & 1 & & & \\
\hline Recovery Resistance & 1 & \multirow{2}{*}{ True } & \multirow{2}{*}{79} & \multirow{2}{*}{26} \\
\hline Smoothing Method & Moving Mean & & & \\
\hline Smoothing Window Size & 60 & \multirow{2}{*}{ False } & \multirow{2}{*}{125} & \multirow{2}{*}{$\mathrm{X}$} \\
\hline Baseline & 40.0 & & & \\
\hline
\end{tabular}

Table C.22: The parameters and confusion matrix for BinarySMO (no filtering) using the Baseline Monitor. 


\begin{tabular}{|c|c|c|c|c|}
\hline Parameter & Value & \multirow{4}{*}{ Predicted Actual } & \multirow{4}{*}{ True } & \multirow{4}{*}{ False } \\
\hline Resistance Method & Fighting & & & \\
\hline Alert Resistance & 9 & & & \\
\hline Recovery Resistance & 10 & & & \\
\hline Tolerance & 1900.0000 & True & 88 & 12 \\
\hline Smoothing Method & Moving Mean & False & 116 & $\mathrm{X}$ \\
\hline Smoothing Window Size & 40 & & & \\
\hline Window Size & 10 & & & \\
\hline
\end{tabular}

Table C.23: The parameters and confusion matrix for SMO (no filtering) using the MSE Monitor.

\begin{tabular}{|c|c|c|c|c|}
\hline Parameter & Value & \multirow{3}{*}{ Predicted Actual } & \multirow[b]{3}{*}{ True } & \multirow[b]{3}{*}{ False } \\
\hline Resistance Method & Continuious & & & \\
\hline Alert Resistance & 1 & & & \\
\hline Recovery Resistance & 8 & \multirow{2}{*}{ True } & \multirow{2}{*}{66} & \multirow{2}{*}{7} \\
\hline Smoothing Method & Moving Mean & & & \\
\hline Smoothing Window Size & 100 & \multirow{2}{*}{ False } & \multirow{2}{*}{138} & \multirow{2}{*}{$\mathrm{X}$} \\
\hline Baseline & 100.0 & & & \\
\hline
\end{tabular}

Table C.24: The parameters and confusion matrix for BPNB (full filtering) using the Baseline Monitor. 


\begin{tabular}{|c|c|c|c|c|}
\hline Parameter & Value & \multirow{3}{*}{ Predicted Actual } & \multirow[b]{3}{*}{ True } & \multirow[b]{3}{*}{ False } \\
\hline Resistance Method & Window & & & \\
\hline Window Size & 15 & & & \\
\hline Control & 2 & \multirow{2}{*}{ True } & \multirow{2}{*}{68} & \multirow{2}{*}{8} \\
\hline Tolerance & 0.7500 & & & \\
\hline Smoothing Method & Moving Mean & \multirow{2}{*}{ False } & \multirow{2}{*}{136} & \multirow{2}{*}{$\mathrm{X}$} \\
\hline Smoothing Window Size & 100 & & & \\
\hline
\end{tabular}

Table C.25: The parameters and confusion matrix for BPNB (full filtering) using the Ratio Monitor.

\begin{tabular}{|c|c|c|c|c|}
\hline Parameter & Value & \multirow{3}{*}{ Predicted Actual } & \multirow[b]{3}{*}{ True } & \multirow[b]{3}{*}{ False } \\
\hline Resistance Method & Continuious & & & \\
\hline Alert Resistance & 2 & & & \\
\hline Recovery Resistance & 2 & \multirow{2}{*}{ True } & 81 & 14 \\
\hline Smoothing Method & Moving Mean & & \multirow{3}{*}{123} & \multirow{3}{*}{$\mathrm{X}$} \\
\hline Smoothing Window Size & 50 & \multirow{2}{*}{ False } & & \\
\hline Baseline & 80.0 & & & \\
\hline
\end{tabular}

Table C.26: The parameters and confusion matrix for SMO (full filtering) using the Baseline Monitor.

\begin{tabular}{|c|c|c|c|c|}
\hline Parameter & Value & \multirow{3}{*}{ Predicted Actual } & \multirow{3}{*}{ True } & \multirow{3}{*}{ False } \\
\hline Resistance Method & Continuious & & & \\
\hline Alert Resistance & 3 & & & \\
\hline Recovery Resistance & 9 & True & 66 & 34 \\
\hline Tolerance & 0.7500 & False & 138 & $\mathrm{X}$ \\
\hline Smoothing Method & None & & & \\
\hline
\end{tabular}

Table C.27: The parameters and confusion matrix for SMO (full filtering) using the Ratio Monitor. 


\begin{tabular}{|c|c|c|c|c|}
\hline Parameter & Value & & & \\
\hline Resistance Method & Fighting & & & \\
\hline Alert Resistance & 9 & Predicted Actual & True & False \\
\hline Recovery Resistance & 9 & \multirow{2}{*}{\begin{tabular}{|r} 
Predicted \\
True
\end{tabular}} & 80 & 0 \\
\hline Smoothing Method & Moving Mean & & & \multirow{3}{*}{$\mathrm{X}$} \\
\hline Smoothing Window Size & 40 & \multirow{2}{*}{ False } & \multirow{2}{*}{124} & \\
\hline Baseline & 60.0 & & & \\
\hline
\end{tabular}

Table C.28: The parameters and confusion matrix for BPNB (no filtering) using the Baseline Monitor.

\begin{tabular}{|c|c|c|c|c|}
\hline Parameter & Value & & & \\
\hline Resistance Method & Continuious & \multirow{2}{*}{ Predicted Actual } & \multirow{2}{*}{ True } & \multirow{2}{*}{ False } \\
\hline Alert Resistance & 3 & & & \\
\hline Recovery Resistance & 10 & True & 58 & 22 \\
\hline Tolerance & 0.8500 & False & 146 & $\mathrm{X}$ \\
\hline Smoothing Method & None & & & \\
\hline
\end{tabular}

Table C.29: The parameters and confusion matrix for SMO (no filtering) using the Ratio Monitor.

\begin{tabular}{|c|c|c|c|c|}
\hline Parameter & Value & & & \\
\hline Resistance Method & Continuious & \multirow{2}{*}{ Predicted Actual } & \multirow{2}{*}{ True } & \multirow{2}{*}{ False } \\
\hline Alert Resistance & 1 & & & \\
\hline Recovery Resistance & 10 & True & 61 & 42 \\
\hline Tolerance & 0.8000 & False & 143 & $\mathrm{X}$ \\
\hline Smoothing Method & None & & & \\
\hline
\end{tabular}

Table C.30: The parameters and confusion matrix for BinaryNaiveBayes (no filtering) using the Ratio Monitor. 


\begin{tabular}{|c|c|c|c|c|}
\hline Parameter & Value & \multirow{3}{*}{ Predicted Actual } & \multirow{3}{*}{ True } & \multirow{3}{*}{ False } \\
\hline Resistance Method & Continuious & & & \\
\hline Alert Resistance & 3 & & & \\
\hline Recovery Resistance & 10 & True & 46 & 12 \\
\hline Tolerance & 0.8500 & False & 158 & $\mathrm{X}$ \\
\hline Smoothing Method & None & & & \\
\hline
\end{tabular}

Table C.31: The parameters and confusion matrix for NaiveBayes (no filtering) using the Ratio Monitor.

\begin{tabular}{|c|c|c|c|c|}
\hline Parameter & Value & & & \\
\hline Resistance Method & Window & \multirow{2}{*}{ Predicted Actual } & \multirow{2}{*}{ True } & \multirow{2}{*}{ False } \\
\hline Window Size & 15 & & & \\
\hline Control & 2 & True & 61 & 41 \\
\hline Tolerance & 0.8500 & False & 143 & $\mathrm{X}$ \\
\hline Smoothing Method & None & & & \\
\hline
\end{tabular}

Table C.32: The parameters and confusion matrix for BinarySMO (full filtering) using the Ratio Monitor.

\begin{tabular}{|c|c|c|c|c|}
\hline Parameter & Value & \multirow{4}{*}{ Predicted Actual } & \multirow{4}{*}{ True } & \multirow{4}{*}{ False } \\
\hline Resistance Method & Continuious & & & \\
\hline Alert Resistance & 1 & & & \\
\hline Recovery Resistance & 10 & & & \\
\hline Tolerance & 0.8500 & True & 62 & 48 \\
\hline Smoothing Method & Moving Mean & False & 142 & $\mathrm{X}$ \\
\hline Smoothing Window Size & 90 & & & \\
\hline Window Size & 10 & & & \\
\hline
\end{tabular}

Table C.33: The parameters and confusion matrix for SMO (full filtering) using the Correlation Monitor. 


\begin{tabular}{|c|c|c|c|c|}
\hline Parameter & Value & & & \\
\hline Resistance Method & Continuious & \multirow{2}{*}{ Predicted Actual } & \multirow{2}{*}{ True } & \multirow{2}{*}{ False } \\
\hline Alert Resistance & 2 & & & \\
\hline Recovery Resistance & 10 & True & 44 & 16 \\
\hline Tolerance & 0.8500 & False & 160 & $\mathrm{X}$ \\
\hline Smoothing Method & None & & & \\
\hline
\end{tabular}

Table C.34: The parameters and confusion matrix for BinarySMO (no filtering) using the Ratio Monitor.

\begin{tabular}{|c|c|c|c|c|}
\hline Parameter & Value & & & \\
\hline Resistance Method & Continuious & \multirow{2}{*}{ Predicted Actual } & \multirow{2}{*}{ True } & \multirow{2}{*}{ False } \\
\hline Alert Resistance & 1 & & & \\
\hline Recovery Resistance & 10 & True & 62 & 59 \\
\hline Tolerance & 0.7500 & False & 142 & $\mathrm{X}$ \\
\hline Smoothing Method & None & & & \\
\hline
\end{tabular}

Table C.35: The parameters and confusion matrix for BPNB (no filtering) using the Ratio Monitor.

\begin{tabular}{|c|c|c|c|c|}
\hline Parameter & Value & \multirow{4}{*}{ Predicted Actual } & \multirow{4}{*}{ True } & \multirow{4}{*}{ False } \\
\hline Resistance Method & Continuious & & & \\
\hline Alert Resistance & 1 & & & \\
\hline Recovery Resistance & 10 & & & \\
\hline Tolerance & 0.8000 & True & 55 & 45 \\
\hline Smoothing Method & Moving Mean & False & 149 & $\mathrm{X}$ \\
\hline Smoothing Window Size & 90 & & & \\
\hline Window Size & 10 & & & \\
\hline
\end{tabular}

Table C.36: The parameters and confusion matrix for BPNB (full filtering) using the Correlation Monitor. 


\begin{tabular}{|c|c|c|c|c|}
\hline Parameter & Value & & & \\
\hline Resistance Method & Fighting & \multirow{2}{*}{ Predicted Actual } & \multirow{2}{*}{ True } & \multirow{2}{*}{ False } \\
\hline Alert Resistance & 10 & & & \\
\hline Recovery Resistance & 9 & True & 84 & 13 \\
\hline Tolerance & 0.8000 & False & 120 & $\mathrm{X}$ \\
\hline Smoothing Method & None & & & \\
\hline
\end{tabular}

Table C.37: The parameters and confusion matrix for BinaryNaiveBayes (full filtering) using the Ratio Monitor.

\begin{tabular}{|l|c|c|c|c|}
\hline Parameter & Value \\
\hline Resistance Method & Continuious & \multicolumn{3}{|c|}{} \\
\hline Alert Resistance & 1 & Actual & True & False \\
\hline Recovery Resistance & 10 & True & 47 & 35 \\
\hline Tolerance & 0.8500 & False & 157 & X \\
\hline Smoothing Method & Moving Mean & & & \\
\hline Smoothing Window Size & 90 & & & \\
\hline Window Size & 10 & & & \\
\end{tabular}

Table C.38: The parameters and confusion matrix for BPNB (no filtering) using the Correlation Monitor. 


\begin{tabular}{|c|c|c|c|c|}
\hline Parameter & Value & \multirow{4}{*}{ Predicted Actual } & \multirow{4}{*}{ True } & \multirow{4}{*}{ False } \\
\hline Resistance Method & Continuious & & & \\
\hline Alert Resistance & 1 & & & \\
\hline Recovery Resistance & 10 & & & \\
\hline Tolerance & 0.8500 & True & 51 & 45 \\
\hline Smoothing Method & Moving Mean & False & 153 & $\mathrm{X}$ \\
\hline Smoothing Window Size & 80 & & & \\
\hline Window Size & 10 & & & \\
\hline
\end{tabular}

Table C.39: The parameters and confusion matrix for BinaryNaiveBayes (full filtering) using the Correlation Monitor.

\begin{tabular}{|c|c|c|c|c|}
\hline Parameter & Value & & & \\
\hline Resistance Method & Continuious & & & \\
\hline Alert Resistance & 1 & \multirow{2}{*}{ Predicted Actual } & \multirow{2}{*}{ True } & \multirow{2}{*}{ False } \\
\hline Recovery Resistance & 9 & & & \\
\hline Tolerance & 0.8500 & True & 38 & 24 \\
\hline Smoothing Method & Moving Mean & False & 166 & $\mathrm{X}$ \\
\hline Smoothing Window Size & 90 & & & \\
\hline Window Size & 10 & & & \\
\hline
\end{tabular}

Table C.40: The parameters and confusion matrix for J48 (full filtering) using the Correlation Monitor. 


\begin{tabular}{|l|c|c|c|c|}
\hline Parameter & Value \\
\hline Resistance Method & Continuious & \multicolumn{3}{|c|}{} \\
\hline Alert Resistance & 1 & Actual & True & False \\
\hline Recovery Resistance & 9 & True & 32 & 19 \\
\hline Tolerance & 0.8500 & False & 172 & X \\
\hline Smoothing Method & Moving Mean & & & \\
\hline Smoothing Window Size & 90 & & & \\
\hline Window Size & 10 & & & \\
\cline { 1 - 2 } & &
\end{tabular}

Table C.41: The parameters and confusion matrix for SMO (no filtering) using the Correlation Monitor.

\begin{tabular}{|c|c|c|c|c|}
\hline Parameter & Value & \multirow{3}{*}{ Predicted Actual } & \multirow[b]{3}{*}{ True } & \multirow[b]{3}{*}{ False } \\
\hline Resistance Method & Continuious & & & \\
\hline Alert Resistance & 10 & & & \\
\hline Recovery Resistance & 1 & \multirow{2}{*}{ True } & \multirow{2}{*}{132} & \multirow{2}{*}{165} \\
\hline Tolerance & 1900.0000 & & & \\
\hline Smoothing Method & None & \multirow{2}{*}{ False } & \multirow{2}{*}{72} & \multirow{2}{*}{$\mathrm{X}$} \\
\hline Window Size & 10 & & & \\
\hline
\end{tabular}

Table C.42: The parameters and confusion matrix for J48 (full filtering) using the MSE Monitor. 


\begin{tabular}{|c|c|c|c|c|}
\hline Parameter & Value & \multirow{3}{*}{ Predicted Actual } & \multirow[b]{3}{*}{ True } & \multirow[b]{3}{*}{ False } \\
\hline Resistance Method & Continuious & & & \\
\hline Alert Resistance & 9 & & & \\
\hline Recovery Resistance & 1 & \multirow{2}{*}{ True } & \multirow{2}{*}{136} & \multirow{2}{*}{165} \\
\hline Tolerance & 1900.0000 & & & \\
\hline Smoothing Method & None & \multirow{2}{*}{ False } & \multirow{2}{*}{68} & \multirow{2}{*}{$\mathrm{X}$} \\
\hline Window Size & 10 & & & \\
\hline
\end{tabular}

Table C.43: The parameters and confusion matrix for BPNB (no filtering) using the MSE Monitor.

\begin{tabular}{|l|c||c|c|c|}
\hline Parameter & Value \\
\hline Resistance Method & Continuious & \multicolumn{3}{|l|}{} \\
\hline Alert Resistance & 1 & Actual & True & False \\
\hline Recovery Resistance & 10 & True & 29 & 26 \\
\hline Tolerance & 0.8500 & False & 175 & X \\
\hline Smoothing Method & Moving Mean & & & \\
\hline Smoothing Window Size & 90 & & & \\
\hline Window Size & 10 & & & \\
\cline { 1 - 2 } &
\end{tabular}

Table C.44: The parameters and confusion matrix for BinaryNaiveBayes (no filtering) using the Correlation Monitor. 


\begin{tabular}{|c|c|c|c|c|}
\hline Parameter & Value & \multirow{4}{*}{ Predicted Actual } & \multirow{4}{*}{ True } & \multirow{4}{*}{ False } \\
\hline Resistance Method & Continuious & & & \\
\hline Alert Resistance & 1 & & & \\
\hline Recovery Resistance & 10 & & & \\
\hline Tolerance & 0.8500 & True & 32 & 39 \\
\hline Smoothing Method & Moving Mean & False & 172 & $\mathrm{X}$ \\
\hline Smoothing Window Size & 50 & & & \\
\hline Window Size & 10 & & & \\
\hline
\end{tabular}

Table C.45: The parameters and confusion matrix for BinarySMO (full filtering) using the Correlation Monitor.

\begin{tabular}{|l|c|c|c|c|}
\hline Parameter & Value \\
\hline Resistance Method & Continuious & \multicolumn{3}{|c|}{} \\
\hline Alert Resistance & 1 & Actual & True & False \\
\hline Recovery Resistance & 10 & True & 32 & 41 \\
\hline Tolerance & 0.8500 & False & 172 & X \\
\hline Smoothing Method & Moving Mean & & & \\
\hline Smoothing Window Size & 50 & & & \\
\hline Window Size & 10 & & & \\
\hline
\end{tabular}

Table C.46: The parameters and confusion matrix for BinarySMO (no filtering) using the Correlation Monitor. 


\begin{tabular}{|c|c|c|c|c|}
\hline Parameter & Value & \multirow{4}{*}{ Predicted Actual } & \multirow{4}{*}{ True } & \multirow{4}{*}{ False } \\
\hline Resistance Method & Continuious & & & \\
\hline Alert Resistance & 1 & & & \\
\hline Recovery Resistance & 8 & & & \\
\hline Tolerance & 0.8500 & True & 22 & 12 \\
\hline Smoothing Method & Moving Mean & False & 182 & $\mathrm{X}$ \\
\hline Smoothing Window Size & 10 & & & \\
\hline Window Size & 10 & & & \\
\hline
\end{tabular}

Table C.47: The parameters and confusion matrix for NaiveBayes (no filtering) using the Correlation Monitor.

\begin{tabular}{|c|c|c|c|c|}
\hline Parameter & Value & \multirow{3}{*}{ Predicted Actual } & \multirow[b]{3}{*}{ True } & \multirow[b]{3}{*}{ False } \\
\hline Resistance Method & Continuious & & & \\
\hline Alert Resistance & 10 & & & \\
\hline Recovery Resistance & 1 & \multirow{2}{*}{ True } & \multirow{2}{*}{162} & \multirow{2}{*}{146} \\
\hline Tolerance & 1900.0000 & & & \\
\hline Smoothing Method & None & \multirow{2}{*}{ False } & \multirow{2}{*}{42} & \multirow{2}{*}{$\mathrm{X}$} \\
\hline Window Size & 10 & & & \\
\hline
\end{tabular}

Table C.48: The parameters and confusion matrix for SMO (full filtering) using the MSE Monitor. 


\begin{tabular}{|c|c|c|c|c|}
\hline Parameter & Value & \multirow{3}{*}{ Predicted Actual } & \multirow[b]{3}{*}{ True } & \multirow[b]{3}{*}{ False } \\
\hline Resistance Method & Continuious & & & \\
\hline Alert Resistance & 10 & & & \\
\hline Recovery Resistance & 1 & True & 153 & 153 \\
\hline Tolerance & 1900.0000 & \multirow{3}{*}{ False } & \multirow{3}{*}{51} & \multirow{2}{*}{$\mathrm{X}$} \\
\hline Smoothing Method & None & & & \\
\hline Window Size & 10 & & & \\
\hline
\end{tabular}

Table C.49: The parameters and confusion matrix for BinaryNaiveBayes (full filtering) using the MSE Monitor.

\begin{tabular}{|c|c|c|c|c|}
\hline Parameter & Value & \multirow{3}{*}{ Predicted Actual } & \multirow[b]{3}{*}{ True } & \multirow[b]{3}{*}{ False } \\
\hline Resistance Method & Continuious & & & \\
\hline Alert Resistance & 10 & & & \\
\hline Recovery Resistance & 1 & True & 163 & 143 \\
\hline Tolerance & 1900.0000 & \multirow{3}{*}{ False } & \multirow{3}{*}{41} & \multirow{3}{*}{$\mathrm{X}$} \\
\hline Smoothing Method & None & & & \\
\hline Window Size & 10 & & & \\
\hline
\end{tabular}

Table C.50: The parameters and confusion matrix for BPNB (full filtering) using the MSE Monitor. 


\begin{tabular}{|c|c|c|c|c|}
\hline Parameter & Value & \multirow{4}{*}{ Predicted Actual } & \multirow{4}{*}{ True } & \multirow{4}{*}{ False } \\
\hline Resistance Method & Continuious & & & \\
\hline Alert Resistance & 10 & & & \\
\hline Recovery Resistance & 1 & & & \\
\hline Smoothing Method & None & True & 194 & 82 \\
\hline Lower Tolerance & 3.0 & False & 10 & $\mathrm{X}$ \\
\hline Upper Tolerance & 3.0 & & & \\
\hline Window Size & 20 & & & \\
\hline
\end{tabular}

Table C.51: The parameters and confusion matrix for BinarySMO (no filtering) using the WindowStdDev Monitor.

\begin{tabular}{|c|c|c|c|c|}
\hline Parameter & Value & \multirow{4}{*}{ Predicted Actual } & \multirow{4}{*}{ True } & \multirow{4}{*}{ False } \\
\hline Resistance Method & Continuious & & & \\
\hline Alert Resistance & 10 & & & \\
\hline Recovery Resistance & 1 & & & \\
\hline Smoothing Method & None & True & 194 & 82 \\
\hline Lower Tolerance & 3.0 & False & 10 & $\mathrm{X}$ \\
\hline Upper Tolerance & 3.0 & & & \\
\hline Window Size & 20 & & & \\
\hline
\end{tabular}

Table C.52: The parameters and confusion matrix for NaiveBayes (no filtering) using the WindowStdDev Monitor. 


\begin{tabular}{|c|c|c|c|c|}
\hline Parameter & Value & \multirow{4}{*}{ Predicted Actual } & \multirow{4}{*}{ True } & \multirow{4}{*}{ False } \\
\hline Resistance Method & Continuious & & & \\
\hline Alert Resistance & 10 & & & \\
\hline Recovery Resistance & 1 & & & \\
\hline Smoothing Method & None & True & 194 & 81 \\
\hline Lower Tolerance & 3.0 & False & 10 & $\mathrm{X}$ \\
\hline Upper Tolerance & 3.0 & & & \\
\hline Window Size & 20 & & & \\
\hline
\end{tabular}

Table C.53: The parameters and confusion matrix for BinaryNaiveBayes (no filtering) using the WindowStdDev Monitor.

\begin{tabular}{|c|c|c|c|c|}
\hline Parameter & Value & & & \\
\hline Resistance Method & Continuious & & & \\
\hline Alert Resistance & 10 & \multirow{2}{*}{ Predicted Actual } & \multirow{2}{*}{ True } & \multirow{2}{*}{ False } \\
\hline Recovery Resistance & 1 & & & \\
\hline Smoothing Method & None & True & 194 & 82 \\
\hline Lower Tolerance & 3.0 & False & 10 & $\mathrm{X}$ \\
\hline Upper Tolerance & 3.0 & & & \\
\hline Window Size & 20 & & & \\
\hline
\end{tabular}

Table C.54: The parameters and confusion matrix for BinarySMO (full filtering) using the WindowStdDev Monitor. 


\begin{tabular}{|c|c|c|c|c|}
\hline Parameter & Value & \multirow{4}{*}{ Predicted Actual } & \multirow{4}{*}{ True } & \multirow{4}{*}{ False } \\
\hline Resistance Method & Continuious & & & \\
\hline Alert Resistance & 10 & & & \\
\hline Recovery Resistance & 1 & & & \\
\hline Smoothing Method & None & True & 194 & 81 \\
\hline Lower Tolerance & 3.0 & False & 10 & $\mathrm{X}$ \\
\hline Upper Tolerance & 3.0 & & & \\
\hline Window Size & 20 & & & \\
\hline
\end{tabular}

Table C.55: The parameters and confusion matrix for SMO (no filtering) using the WindowStdDev Monitor.

\begin{tabular}{|c|c|c|c|c|}
\hline Parameter & Value & \multirow{4}{*}{ Predicted Actual } & \multirow{4}{*}{ True } & \multirow{4}{*}{ False } \\
\hline Resistance Method & Continuious & & & \\
\hline Alert Resistance & 10 & & & \\
\hline Recovery Resistance & 1 & & & \\
\hline Smoothing Method & None & True & 194 & 79 \\
\hline Lower Tolerance & 3.0 & False & 10 & $\mathrm{X}$ \\
\hline Upper Tolerance & 3.0 & & & \\
\hline Window Size & 20 & & & \\
\hline
\end{tabular}

Table C.56: The parameters and confusion matrix for J48 (full filtering) using the WindowStdDev Monitor. 


\begin{tabular}{|c|c|c|c|c|}
\hline Parameter & Value & \multirow{4}{*}{ Predicted Actual } & \multirow{4}{*}{ True } & \multirow{4}{*}{ False } \\
\hline Resistance Method & Continuious & & & \\
\hline Alert Resistance & 10 & & & \\
\hline Recovery Resistance & 1 & & & \\
\hline Smoothing Method & None & True & 194 & 81 \\
\hline Lower Tolerance & 3.0 & False & 10 & $\mathrm{X}$ \\
\hline Upper Tolerance & 3.0 & & & \\
\hline Window Size & 20 & & & \\
\hline
\end{tabular}

Table C.57: The parameters and confusion matrix for BPNB (no filtering) using the WindowStdDev Monitor.

\begin{tabular}{|c|c|c|c|c|}
\hline Parameter & Value & & & \\
\hline Resistance Method & Continuious & & & \\
\hline Alert Resistance & 10 & \multirow{2}{*}{ Predicted Actual } & \multirow{2}{*}{ True } & \multirow{2}{*}{ False } \\
\hline Recovery Resistance & 1 & & & \\
\hline Smoothing Method & None & True & 195 & 80 \\
\hline Lower Tolerance & 3.0 & False & 9 & $\mathrm{X}$ \\
\hline Upper Tolerance & 3.0 & & & \\
\hline Window Size & 20 & & & \\
\hline
\end{tabular}

Table C.58: The parameters and confusion matrix for BPNB (full filtering) using the WindowStdDev Monitor. 


\begin{tabular}{|c|c|c|c|c|}
\hline Parameter & Value & \multirow{4}{*}{ Predicted Actual } & \multirow{4}{*}{ True } & \multirow{4}{*}{ False } \\
\hline Resistance Method & Continuious & & & \\
\hline Alert Resistance & 10 & & & \\
\hline Recovery Resistance & 1 & & & \\
\hline Smoothing Method & None & True & 194 & 77 \\
\hline Lower Tolerance & 3.0 & False & 10 & $\mathrm{X}$ \\
\hline Upper Tolerance & 3.0 & & & \\
\hline Window Size & 20 & & & \\
\hline
\end{tabular}

Table C.59: The parameters and confusion matrix for BinaryNaiveBayes (full filtering) using the WindowStdDev Monitor.

\begin{tabular}{|c|c|c|c|c|}
\hline Parameter & Value & & & \\
\hline Resistance Method & Continuious & & & \\
\hline Alert Resistance & 10 & \multirow{2}{*}{ Predicted Actual } & \multirow{2}{*}{ True } & \multirow{2}{*}{ False } \\
\hline Recovery Resistance & 1 & & & \\
\hline Smoothing Method & None & True & 194 & 78 \\
\hline Lower Tolerance & 3.0 & False & 10 & $\mathrm{X}$ \\
\hline Upper Tolerance & 3.0 & & & \\
\hline Window Size & 20 & & & \\
\hline
\end{tabular}

Table C.60: The parameters and confusion matrix for SMO (full filtering) using the WindowStdDev Monitor. 


\section{Appendix D}

\section{Full Training Set}

\begin{tabular}{|l|l|}
\hline complaint & I hate netflix sometimes \\
\hline complaint & Fuck netflix \\
\hline complaint & Wat the fuck is a fuck n netflix ? \\
\hline watching & Netflix movies all dayyy \\
\hline neutral & !!!! THEY HAVE AAAHH! REAL MONSTERS ON NETFLIX! FUCK YEAH. \\
\hline complaint & NETFLIX Y U HATE ME? \\
\hline complaint & Netflix is so trash wtf. \\
\hline neutral & Netflix stoped messing up \#hypetweet \\
\hline complaint & ugh...netflix is THEE worse \\
\hline complaint & I hate when netflix fucks up! \\
\hline snafu & I hate when netflix fucks up! \\
\hline snafu & Netflix is down. EVERYBODY PANIC \\
\hline snafu & Netflix and Xbox not getting along. \\
\hline response & RT @Nat3Th3Gr3at: myy netflix isn't workin.. : ( \\
\hline snafu & RT @Nat3Th3Gr3at: myy netflix isn't workin.. : ( \\
\hline snafu & Netflix isn’t working!o:i \\
\hline snafu & $\begin{array}{l}\text { Netflix... can access movie but can't play.... what an evil game they r playing. Anyone else having this } \\
\text { problem? \#netflix }\end{array}$ \\
\hline
\end{tabular}




\begin{tabular}{|c|c|}
\hline snafu & $\begin{array}{l}\text { Dear @netflix and @Xbox and @XboxSupport please fix yoru Netflix crap.... I was in the middle of } \\
\text { finishing up season } 4 \text { of My Name is Earl! }\end{array}$ \\
\hline snafu & Netflix, Y U NO WORK? \\
\hline complaint & $\begin{array}{l}\text { @sh33k360 Yeah. I can access my queue, but when I hit play, it goes back to the list after about } 10 \\
\text { seconds of loading. \#netflix }\end{array}$ \\
\hline snafu & $\begin{array}{l}\text { @sh33k360 Yeah. I can access my queue, but when I hit play, it goes back to the list after about } 10 \\
\text { seconds of loading. \#netflix }\end{array}$ \\
\hline snafu & Netflix won't stream WTF Mavs dont play for another 40 mins \\
\hline snafu & @xboxsupport please fix netflix ASAP! K, thnks! \\
\hline snafu & $\begin{array}{l}\text { @ VballChickPMS I've got a few pings via Xbox Xperts asking the same thing. Seems Netflix is having } \\
\text { problems. }\end{array}$ \\
\hline snafu & $\begin{array}{l}\text { @XboxSupport any reports of Netflix issues tonight in Canada?I try to start a movie, get } 4 \text { "quality" } \\
\text { bars, then back to menu screen. }\end{array}$ \\
\hline snafu & Anybody else having trouble with Netflix right now? \\
\hline snafu & Is anyone else having issues with Netflix via Xbox? iPhone \& PC work fine. \\
\hline snafu & Why the Eff is my Netflix acting stupid \\
\hline snafu & Damn NetFlix via Xbox 360 DOWN! (Bbm Sad Face) \\
\hline snafu & @netflix not loading vids on xbox \\
\hline response & $\begin{array}{l}\text { @thornlordslady apparently it's just xbox } 360 \text { and netflix is working on a fix.saw the tweet from them } \\
\text { about } 5 \text { mins ago. }\end{array}$ \\
\hline snafu & $\begin{array}{l}\text { @thornlordslady apparently it's just xbox } 360 \text { and netflix is working on a fix.saw the tweet from them } \\
\text { about } 5 \text { mins ago. }\end{array}$ \\
\hline snafu & $\begin{array}{l}\text { Hmm...been having issues myself lately.Just blamed TWC for it :-/RT @TheSocialGamer: Is something } \\
\text { going on with \#Netflix for \#Xbox360? }\end{array}$ \\
\hline snafu & Grrr xbox is not playing nice with netflix right now, yet my ps3 works just fine. Buuuhh \\
\hline snafu & Is Netflix on 360 not working for anyone else right now? \\
\hline complaint & netflix is screwing up...\#fml \\
\hline snafu & netflix is screwing up...\#fml \\
\hline snafu & Looks like it is not just me, Netflix is not working on my Xbox. \\
\hline snafu & Why isn't my netflix working? \\
\hline
\end{tabular}




\begin{tabular}{|c|c|}
\hline snafu & \#netflix fix your shit! \\
\hline snafu & Netflix isn’t working $=($ \\
\hline snafu & Is \#netflix on Xbox down for anyone else? \\
\hline snafu & @XboxSupportWhere is netflix on the dashboard?? \\
\hline snafu & Y Netflix actn all dumb nd shit! \\
\hline snafu & $\begin{array}{l}\text { Dear @netflix, I've been using the service on my Xbox for a few hours now. All of a sudden I can't play } \\
\text { anything. Please fix. Thank you. }\end{array}$ \\
\hline snafu & $\begin{array}{l}\text { @xboxsupport i cant get my netflix to work. When i start a movie it starts normal like its loadibg then } \\
\text { goes back to the main screen }\end{array}$ \\
\hline snafu & $\begin{array}{l}\text { Anyone else having trouble getting netflix work on the xbox? Mine kicks out after determining my picture } \\
\text { quality to be HD. }\end{array}$ \\
\hline snafu & why isn't my netflix working :( \\
\hline snafu & $\begin{array}{l}\text { NO NETFLIX UGH NOW HOW ARE WE GOIN TO WATCH MOVIES.....jacked up stuff yo gosh smh } \\
\text { **folds arms and pouts** }\end{array}$ \\
\hline snafu & @XboxSupportYo where can i find netflix? \\
\hline snafu & $\begin{array}{l}\text { @Netflix I can't watch anything on my } 360 \text { says it can't contact, my friend are having the same problem, } \\
\text { what's up? }\end{array}$ \\
\hline snafu & I'm really mad Netflix on xbox isn't working $¿: O$ \\
\hline snafu & myy netflix isn’t workin.. : ( \\
\hline snafu & Netflix on Xbox Live having issues streaming tonight. Boo! \\
\hline snafu & OMFG FUCK YOU NETFLIX! Being hella laggy and shit. ugh!! \\
\hline complaint & Holy fucking slow \#netflix \\
\hline snafu & Holy fucking slow \#netflix \\
\hline snafu & What's wrong with \#Netflix \\
\hline snafu & need help my netflix is not working on my xbox 360 \\
\hline snafu & Dammit \#Xbox and/or @netflix! Why did you suddenly decide to stop working?!?! \\
\hline response & @evilly_innocent i am going to figure out what the heck happened to my netflix $i \cdot i$ \\
\hline snafu & @evilly_innocent i am going to figure out what the heck happened to my netflix $i \cdot i$ \\
\hline watching & \#netflix chainsaw massacre \\
\hline watching & Kangaroo Jack on Netflix - sick daughter's choice \\
\hline
\end{tabular}




\begin{tabular}{|c|c|}
\hline watching & First day of dead week- watching Criminal Minds... :) Yay for netflix! \\
\hline complaint & Bumass netflix -_-- \\
\hline watching & Death at a funeral \#netflix \#ps3 \\
\hline response & RT @SamuelDeLaGetto: Watchu watchin? Im watchin "Donnie Darko" RT @MarcusGdaCeleb: \#Netflix \\
\hline watching & RT @SamuelDeLaGetto: Watchu watchin? Im watchin "Donnie Darko" RT @MarcusGdaCeleb: \#Netflix \\
\hline snafu & 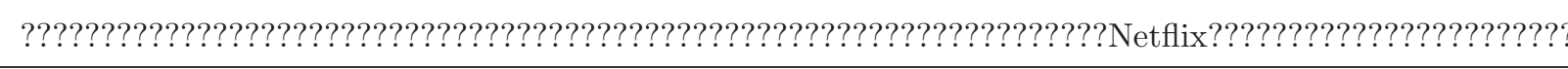 \\
\hline watching & Coughing up hairballs (not literally) and watching netflix on XBL *sighs* I hate being sick! \\
\hline happy & Netflix;3 \\
\hline watching & Watching \#aaahhrealmonsters on \#netflix! Hell yessss \\
\hline complaint & Stupid fucking PS3 and stupid fucking Netflix. \#mallorieproofed \\
\hline snafu & Stupid fucking PS3 and stupid fucking Netflix. \#mallorieproofed \\
\hline complaint & Fuck netflix \\
\hline complaint & Stupid, stupid Netflix. \\
\hline watching & Watchin netflix \\
\hline complaint & Netflix suxs \\
\hline complaint & NetFlix Bitch! \\
\hline complaint & Ugh, fucking Netflix fucking up. \\
\hline snafu & Ugh, fucking Netflix fucking up. \\
\hline complaint & ughh stupid netflix \\
\hline complaint & \#stupid netflix \\
\hline complaint & sorry, i hate netflix. \\
\hline complaint & \#netflix wtf \\
\hline complaint & WTF \#netflix!? \\
\hline complaint & Ugh I hate netflix. \\
\hline complaint & WTF Netflix \\
\hline complaint & OMFG WTF NETFLIX \\
\hline refuse to rate & en serio, QUIERO pagar por algo como Netflix, DEJARME pagar \\
\hline refuse to rate & Contratar Netflix o no contratar Netflix, ese es el dilema... \\
\hline complaint & Ughh wtf netflix!!!! \\
\hline
\end{tabular}




\begin{tabular}{|c|c|}
\hline response & $\begin{array}{l}\text { Also @cbye is it you or one of your other Netflix friends watching Party Down? If it isn't you I suggest } \\
\text { you start immediately! }\end{array}$ \\
\hline watching & $\begin{array}{l}\text { Also @cbye is it you or one of your other Netflix friends watching Party Down? If it isn't you I suggest } \\
\text { you start immediately! }\end{array}$ \\
\hline media & $\begin{array}{l}\text { http://bit.ly/92a4un i- Online Petition to STOP Internet Bandwidth Overage Charges in Shaw's response } \\
\text { to Netflix vs Video On Demand }\end{array}$ \\
\hline happy & off to bed netflix to lull me to sleep ¡3 \\
\hline neutral & Chillin at home with Netflix and a home cooked meal! \\
\hline neutral & $\begin{array}{l}\text { "The Wild and Wonderful Whites of West Virginia" kept Karly up well past } 11 \text { on a weeknight. Netflix } \\
\text { it to see life lived without a tomorrow. }\end{array}$ \\
\hline response & @DrumMajorsATL What it do my nigga i'm just cooling it watching netflix,lol what u up too \\
\hline watching & @DrumMajorsATL What it do my nigga i'm just cooling it watching netflix,lol what u up too \\
\hline refuse to rate & No es jevy eso?RT @BackHand29: @BaBy_FrEsH103 oye chekeate SPARTICUS en Netflix pa k te cure \\
\hline media & $\begin{array}{l}\text { The Mobile Tsunami is Near: Blame Netflix and Apple: Cisco anticipates that mobile network-connected } \\
\text { tablets wil... http://bit.ly/i8Fvmv }\end{array}$ \\
\hline complaint & @enriqueztwb the real question is when is CSI getting to Netflix Streaming? that is the REAL question. \\
\hline neutral & @enriqueztwb the real question is when is CSI getting to Netflix Streaming? that is the REAL question. \\
\hline response & @enriqueztwb the real question is when is CSI getting to Netflix Streaming? that is the REAL question. \\
\hline neutral & $\begin{array}{l}\text { @friedgreenhalos yep. netflix instant streaming. I love Christopher Lloyd and Michael Jeter (RIP Michael } \\
\text { Jeter). }\end{array}$ \\
\hline response & $\begin{array}{l}\text { @friedgreenhalos yep. netflix instant streaming. I love Christopher Lloyd and Michael Jeter (RIP Michael } \\
\text { Jeter). }\end{array}$ \\
\hline refuse to rate & \#nw waterworld with kev cosner....ill shit \#netflix \\
\hline complaint & netflix keeps taking little things i like about the site away...Why? \\
\hline complaint & And netflix is broken. Why is this happening to me. \\
\hline snafu & And netflix is broken. Why is this happening to me. \\
\hline media & $\begin{array}{l}\text { it's like the netflix challenge but with lives instead of movies!RT @timoreilly: } \$ 3 \text { Million Prize Offered... } \\
\text { http://bit.ly/hyRzHn }\end{array}$ \\
\hline response & $\begin{array}{l}\text { it's like the netflix challenge but with lives instead of movies!RT @timoreilly: } \$ 3 \text { Million Prize Offered... } \\
\text { http://bit.ly/hyRzHn }\end{array}$ \\
\hline
\end{tabular}




\begin{tabular}{|c|c|}
\hline media & The Mobile Tsunami Is Near: Blame Netflix and Apple http://feeds.nytimes.com/click.phdo?i=c959df9634 \\
\hline media & The Mobile Tsunami Is Near: Blame Netflix and Apple http://feeds.nytimes.com/click.phdo?i=c959df9634 \\
\hline watching & Bouts to browse through my \#Netflix on my 360 see wht I wanna watch \\
\hline neutral & @sarahsteinmeier I got an email from netflix alerting me that it will be arriving tomorrow! I am excite. \\
\hline response & @ sarahsteinmeier I got an email from netflix alerting me that it will be arriving tomorrow! I am excite. \\
\hline media & Thanks to the recommendation of @superbetch I watch Netflix on my phone almost every day :) \\
\hline watching & Thanks to the recommendation of @superbetch I watch Netflix on my phone almost every day :) \\
\hline neutral & Boondocks Saints 2. Netflix \#chillmode \\
\hline watching & Boondocks Saints 2. Netflix \#chillmode \\
\hline media & $\begin{array}{l}\text { Last minute again RT @boxee: Netflix App works, but is still "coming soon" due to strict security } \\
\text { requirements - http://bit.ly/dRIoJv }\end{array}$ \\
\hline response & $\begin{array}{l}\text { Last minute again RT @boxee: Netflix App works, but is still "coming soon" due to strict security } \\
\text { requirements - http://bit.ly/dRIoJv }\end{array}$ \\
\hline neutral & What you missed on Netflix Movie Night: http://www.youtube.com/watch?v=X7UQyerHXB0 \\
\hline response & @DstEeZNLikeWOAH netflix just sent me that shit! \\
\hline neutral & $\begin{array}{l}\text { @DirectTV - yes RED was great. I got it from @Netflix. So STOP blasting out the AD at such a high } \\
\text { vol my NEIGHBORS know U have it! \#juststop }\end{array}$ \\
\hline refuse to rate & Netflix. Nigga.((: \\
\hline watching & Let's see what's on Netflix .. \\
\hline neutral & $\begin{array}{l}\text { Perusing Netflix titles on Apple TV sucks unless you have perfect vision or sit } 2 \text { inches from the TV. } \\
\text { Roku gets it right. }\end{array}$ \\
\hline neutral & Netflix streaming a history of the Monarchy, a nice whiskey glow and still, I have writers block... \\
\hline neutral & How do you get Netflix ?? \\
\hline complaint & Netflix is being so slow \#hurryup \\
\hline happy & @zachgalifianakis ur comedy on netflix is \#bomb \\
\hline response & @zachgalifianakis ur comedy on netflix is \#bomb \\
\hline neutral & What did I watch to make Netflix think I like foreign buddy flicks? \\
\hline media & The Mobile Tsunami Is Near: Blame Netflix and Apple http://nyti.ms/i3Wgv3 \\
\hline happy & Netflix might be the best invention \\
\hline watching & multitaskin lol doin work $\mathrm{n}$ watchin Exam on netflix \\
\hline
\end{tabular}




\begin{tabular}{|c|c|}
\hline neutral & $\begin{array}{l}\text { anyone know of a really good, really happy fluffy movie that might be on netflix... ? at all? or anything } \\
\text { at all? i'm so boowooored! }\end{array}$ \\
\hline watching & I'm not watching Secret Life of American Teenager on netflix o_O \#lies \\
\hline happy & UK Skins on Netflix. Yes. \\
\hline happy & I need NetFlix in my life asap \\
\hline media & $\begin{array}{l}\text { Amazon Prime members to get unlimited video streaming, a la Netflix? http://t.co/Ox2NZtn via @dig- } \\
\text { italtrends }\end{array}$ \\
\hline media & $\begin{array}{l}\text { So excited! RT @boxee: Netflix App works, but is still "coming soon" due to strict security requirements } \\
\text { - http://bit.ly/dRIoJv }\end{array}$ \\
\hline complaint & NETFLIX pisses me off when it doesnt work correctly \\
\hline snafu & NETFLIX pisses me off when it doesnt work correctly \\
\hline neutral & Its Netflix tiiiiiime! What film should I watch? I need some suggestions. . . \\
\hline complaint & @Morena485 I'm not terribly impressed with Netflix and their selection of movies. :/ \\
\hline complaint & man, this Shit sucks on tha computer. \#netflix \\
\hline watching & $\begin{array}{l}\text { watchinggg avatar, the last air bender with my sleeping brother on netflix! haha soo muchh for watching } \\
\text { tv together \#sleepyhead }\end{array}$ \\
\hline complaint & Why is netflix online mad limited though? \\
\hline happy & thank goodness for netflix... bones marathon by maseff! \\
\hline response & $\begin{array}{l}\text { @ joshuabier Netflix queue: Day After Tomorrow, 2012, The Shining, Alive, Fargo, and Snow White and } \\
\text { the } 7 \text { Dwarfs. }\end{array}$ \\
\hline neutral & I don't think I'd ever leave the house if I got Netflix. \\
\hline watching & Everyone's sleep. Just wanted to watch @netflix \& its down...so now im officially bored. \\
\hline happy & Damn 2mor is a PERFECT day 4 layin up \& watch netflix smh \\
\hline neutral & @PaprChasNMahni lol I'm watching it now on netflix \\
\hline watching & @PaprChasNMahni lol I'm watching it now on netflix \\
\hline response & @riskyrae get netflix for free for a month. That's how I got Josh addicted. \\
\hline watching & Watching Gamer on Netflix. \\
\hline neutral & If you haven't watched Spartacus on @netflix you're LOSING majorly. \\
\hline happy & So @netflix is amazing \\
\hline
\end{tabular}




\begin{tabular}{|c|c|}
\hline media & $\begin{array}{l}\text { Netflix shares jolted: In addition to streaming movies, Amazon may look to strike a partnership with } \\
\text { Coinstar, w... http://bit.ly/fW12pq }\end{array}$ \\
\hline watching & Going to watch a movie on netflix, "Julie \& Julia". Can't sleep now after that fat nap. \\
\hline watching & $\begin{array}{l}\text { i need to watch something semi-boring instead of party movies on netflix, party related anything makes } \\
\text { me wanna stay up all night! }\end{array}$ \\
\hline watching & Just tried out Netflix and watched Broken Embraces by Pedro Almodovar, Loved it! \\
\hline neutral & $\begin{array}{l}\text { This history paper is going a lot slower than I first imagined, thanks to discovering Desperate Housewives } \\
\text { on Netflix stream-on-demand. }\end{array}$ \\
\hline response & $\begin{array}{l}\text { RT @Lupintheone2011@RonGreco it can snow all it wants as long as it don't mess with my netflix } \\
\text { streaming }\end{array}$ \\
\hline neutral & found harry and the hendersons on netflix \\
\hline watching & $\begin{array}{l}\text { I haven't watched a movie on \#Netflix in awhile now... think i will check it out right now } n \text { find something } \\
\text { to watch. }\end{array}$ \\
\hline neutral & Thinkin bout gettin a xbox just so I could get Netflix on my tv lol \\
\hline neutral & netflix changed their instant playing set up. \\
\hline media & $\begin{array}{l}\text { Safeway Inc : Option Skews - Relatively Heavy Put Activity on Netflix Inc., Costco Wholesale ... - } \\
\text { Schaeffers http://uxp.in/27814953 }\end{array}$ \\
\hline happy & @netflix the craft. Girlfriend loves the crap out of that movie \\
\hline neutral & @ netflix the craft. Girlfriend loves the crap out of that movie \\
\hline neutral & I imagine Netflix streaming is getting a workout these days... \\
\hline neutral & I need to get netflix again! Idk what happened in bones, dexter, weeds, and nip/tuck! \\
\hline complaint & $\begin{array}{l}\text { So wanted to watch wizards of waverly place the movie So I turned it on on Netflix and Wendy wu } \\
\text { homecoming warrior came on. What the heck?! }\end{array}$ \\
\hline neutral & @BeehiveBlogBoth good movies.I think I'll put on the netflix list. \\
\hline response & @BeehiveBlogBoth good movies.I think I'll put on the netflix list. \\
\hline response & $\begin{array}{l}\text { I've been watching a lot of @ABCFgreek on Netflix, and the more I do, the more I realize I need a } \\
\text { @scottmfoster (Cappie) for myself! Haha. }\end{array}$ \\
\hline complaint & Hey Netflix! Just mail me my dang movie already! Geeesh! \\
\hline neutral & $\begin{array}{l}\text { There'll be time enough for all the movies in my NetFlix queue, right now I want keep Fringe-ing by } \\
\text { starting season } 2 .\end{array}$ \\
\hline
\end{tabular}




\begin{tabular}{|c|c|}
\hline neutral & Watching a random movie on netflix and eating some food(: \\
\hline watching & Watching a random movie on netflix and eating some food(: \\
\hline happy & Finally watching Ponyo. So happy to have netflix again. \\
\hline watching & Finally watching Ponyo. So happy to have netflix again. \\
\hline happy & Netflix in class best class ever \\
\hline media & $\begin{array}{l}\text { Confirmed: Netflix App Available Exclusively On Future Qualcomm Smartphones, Starting With The } \\
\text { LG Revolution http://bit.ly/i4tKyO }\end{array}$ \\
\hline watching & Watchin the A-Team....shit is hilarious \#Netflix \\
\hline happy & $\begin{array}{l}\text { Awesome! @netflix finally got my Valentine's Day gift from @netflix. They started streaming all } 9 \text { seasons } \\
\text { of \#Scrubs }\end{array}$ \\
\hline happy & so happy i spent my day watching documentaries on Netflix \\
\hline media & $\begin{array}{l}\text { Qualcomm promises Netflix streaming support on 'future Android d... [Engadget Mobile] } \\
\text { http://bzbx.us/PAs \#Netflix \#Qualcomm via buzzbox.com }\end{array}$ \\
\hline media & $\begin{array}{l}\text { Confirmed: Netflix } \\
\text { http://f.ast.ly/VyuxF }\end{array}$ \\
\hline media & Video: The Next Snapdragons Can Stream Netflix on Android Phones [Video] http://goo.gl/fb/6vAol \\
\hline media & Video: The Next Snapdragons Can Stream Netflix on Android Phones [Video] http://goo.gl/fb/1Ii3g \\
\hline happy & $\begin{array}{l}\text { I \#love my \#netflix for the \#wii :) its perfect for lazy ppl like me who hate getting up to change my } \\
\text { \#familyguy dvd }\end{array}$ \\
\hline media & Video: The Next Snapdragons Can Stream Netflix on Android Phones [Video]: ... http://bit.ly/fsmPsN \\
\hline media & Video: The Next Snapdragons Can Stream Netflix on Android Phones [Video] http://dlvr.it/Gk8jh \\
\hline media & $\begin{array}{l}\text { \#Tech \#News: Video: The Next Snapdragons Can Stream Netflix on Android Phones [Video] } \\
\text { http://dlvr.it/Gk8X3 (via Gizmodo) }\end{array}$ \\
\hline neutral & @Karlemilymr nothin home relaxinb, eatin \& about 2 watch a movie on netflix hbu? \\
\hline media & Video: The Next Snapdragons Can Stream Netflix on Android Phones [Video] http://goo.gl/fb/J5eXR \\
\hline neutral & I got half a mind to take another vacation say stay home and watch netflix all day \\
\hline watching & $\begin{array}{l}\text { Still up watching "The Decent 2" on Netflix. I don't mind this rain though cause my stupid car needed } \\
\text { a washing. }\end{array}$ \\
\hline media & R11 Boxee Box gains Netflix support http://nxy.in/tqhav \#techworld \\
\hline happy & Online netflix brings me so much happiness. \\
\hline
\end{tabular}




\begin{tabular}{|c|c|}
\hline media & $\begin{array}{l}\text { Video: The Next Snapdragons Can Stream Netflix on Android Phones [Video]: [GIZ]Those upcoming } \\
\text { Snap... http://tinyurl.com/4vnrw5u }\end{array}$ \\
\hline media & $\begin{array}{l}\text { Video: The Next Snapdragons Can Stream Netflix on Android Phones [Video] (Gizmodo) } \\
\text { http://goo.gl/fb/sESlM }\end{array}$ \\
\hline media & $\begin{array}{l}\text { Apple will take } 30 \% \text { http://tonight.newestheadlines.com/apple-will-take-30-of-subscription-fees-from- } \\
\text { content-based-apps-like-hulu-netflix-2/ }\end{array}$ \\
\hline media & Video: The Next Snapdragons Can Stream Netflix on Android Phones [Video] http://sns.ly/kdbEy1 \\
\hline media & Netflix Now Available Through Facebook - http://bit.ly/ffpBHH - [Geeky Gadgets] \\
\hline media & Netflix Now Available Through Facebook http://goo.gl/fb/gXSiQ \\
\hline media & Netflix Now Available Through Facebook http://bit.ly/eoobW1 \#tech \#apple \#web \\
\hline media & $\begin{array}{l}\text { Netflix was established in 1997, and they offer the ability to rent DVDs online via the Internet. I initially } \\
\text { joined... http://dlvr.it/GkCrb }\end{array}$ \\
\hline media & $\begin{array}{l}\text { Morgan Stanley Downgraded Netflix To EW From OW, Maintained } \$ 245 \text { PT (NFLX): Feb 15, } 2011 \\
\text { (SmarTrend News Watch v... http://bit.ly/hZQyE6 }\end{array}$ \\
\hline media & $\begin{array}{l}\text { Netflix the most engaging online video destination in US: Last month saw the arrival of newcomers to } \\
\text { the top ten... http://bit.ly/dUW } 4 \mathrm{vY}\end{array}$ \\
\hline media & $\begin{array}{l}\text { Netflix arrives on the Boxee Box: Boxee's Avner Ronen has announced that TV shows and movies } \\
\text { streaming instantly... http://bit.ly/getN8z }\end{array}$ \\
\hline happy & Netflix :) \\
\hline media & Video: The Next Snapdragons Can Stream Netflix on Android Phones [Video] http://dld.bz/NhT6 \\
\hline complaint & Y these netflix movies keep stopin an sayin retrieving \#ntheway \\
\hline snafu & Y these netflix movies keep stopin an sayin retrieving \#ntheway \\
\hline happy & NETFLIX;3 \\
\hline happy & @SUGARHEEL Hell Yea!! Shit I'm in Flint!! Andddd I got Netflix! Lmao! \\
\hline watching & @alLOVEson_ im watchn it on netflix loll \\
\hline complaint & Ugh Fuck you Netflix. \\
\hline happy & netflix works yay!!! \\
\hline watching & layin down watchin movies on netflix, bored ass hell \\
\hline snafu & .....is netflix fuckin up? \\
\hline media & Is Facebook Sounding the Netflix Death Knell? http://bit.ly/fsm7ZV \\
\hline
\end{tabular}




\begin{tabular}{|c|c|}
\hline happy & Damn I luv Netflix \\
\hline happy & Netflix gettin hip \\
\hline watching & Watching this documentary about war in Afganistan on Netflix. Scary \& sad... :/ \\
\hline complaint & Tryna get dis netflix on my damn ps3 \\
\hline complaint & netflix always fucks me in the ass \\
\hline happy & \#netflix Yeah! \\
\hline happy & Netflix rules. \\
\hline happy & NetFlix FTW \\
\hline watching & Watchin kevin hart on netflix...shit never gets old haha \\
\hline happy & Netflix $=)$ \\
\hline watching & watching heroes on netflix.....\#addicted \\
\hline complaint & @netflix \#netflix :( \\
\hline snafu & Netflix is fucking up WTF? \\
\hline watching & @nicoleka8701 just watchin netflix and being bored lol \\
\hline complaint & Netflix wtf \\
\hline complaint & Netflix is Soooo stupid \\
\hline watching & watchin more hey arnold on netflix lmao \\
\hline watching & Watching anger management on netflix cause I need dis shit lol \\
\hline snafu & yooooo netflix is playing games right now ughhh \\
\hline complaint & $@$ BrentButt Netflix sux! \\
\hline watching & arrested development streaming netflix. yah. \\
\hline happy & netflix;3 \\
\hline happy & @netflix YAYYYY \\
\hline happy & Netflix is my bestfriend!!!!! Lol \\
\hline happy & \#Netflix :) \\
\hline happy & OHHHHHHHHH!!!!!! BLUESTREAK IS ON NETFLIX!!!!!!!! HELL YEAH!!! \\
\hline happy & Netflix!;3 \\
\hline complaint & Netflix is honestly PISSING me off. \\
\hline happy & Netflix FTW \\
\hline watching & I'm watching Monsters on NetFlix, so pumped \#aliens \#monsters \#scifi \#horror \\
\hline
\end{tabular}




\begin{tabular}{|c|c|}
\hline happy & OMFG, Netflix has Invader Zim! \\
\hline watching & \#NowWatching Black Snake Moan \#Netflix \\
\hline happy & Wooooo we got netflix!! \\
\hline happy & Addicted to Netflix! \#lol \\
\hline snafu & smh sad as hel...l netflixnot working \\
\hline snafu & Tryna fix dis netflix is pissin me off \\
\hline snafu & ugh. NETFLIX WHY ARE YOU FUCKING UP. \\
\hline watching & Watching "Death at a Funeral". \#Netflix \\
\hline complaint & okay netflix kinda sucks \\
\hline complaint & Netflix instant queue movies suck... \\
\hline happy & Netflix is a hell of a drug \\
\hline happy & Netflix ... WOO! \\
\hline complaint & Netflix sucks \\
\hline happy & Wheeee Downton Abbey on Netflix! \\
\hline watching & Watchin Death At A Funeral on Netflix lmfao. \\
\hline happy & Netflix da shit \\
\hline happy & @netflix is the shizz!! \\
\hline snafu & slow azz internet, cant even watch netflix \\
\hline snafu & \#netflix wont work!!! UGH!!!!!! \\
\hline happy & I'm addicted to netflix. \\
\hline happy & Netflix is bomb \\
\hline happy & $\begin{array}{l}\text { @ danieltosh haha dude is cracking me up watching his shit on netflix I'm like pissing my pants about to } \\
\text { run outa underwear }\end{array}$ \\
\hline watching & \#Watchin \#Reaper on NetFlix \\
\hline complaint & this Netflix shit suck \\
\hline happy & Netflix is fucking awesome. \\
\hline happy & netflix timee! \\
\hline happy & I loveeee Netflix. \\
\hline happy & Hell yeahhh got connection again! \#Netflix ; 33 \\
\hline snafu & maaaan ma netflix is effin up \\
\hline
\end{tabular}




\begin{tabular}{|c|c|}
\hline happy & $\begin{array}{l}\text { AWWWWWWW SHIT LATE NIGHT RAGE AGAINST THE MACHINE =)AND THE NETFLIX } \\
\text { HAS DEXTER PLAYING!!!!!!!!! MOSH PIT N E ONE??? }\end{array}$ \\
\hline happy & addicted to netflix... \\
\hline happy & Just got netflix :) hell yeaaaa \\
\hline complaint & Netflix sucks \\
\hline watching & Watching Prison Break in school on \#Netflix? \#addicted? I think not \\
\hline complaint & Damnit! Season 2 of Californication isn't on Netflix instant :( \\
\hline complaint & RT @EXOTIC1988: Netflix kinda suxx's \\
\hline complaint & Netflix kinda suxx's \\
\hline happy & Sesame street on netflix.Yay... something different to watch.I've had my fill of Bob the Builder.;) \\
\hline happy & Omg "Dorian Gray" is on instant on Netflix. Yesssss! Ben Barnes is so, so very attractive. \\
\hline happy & $\begin{array}{l}\text { Just discovered Netflix has added CLEOPATRA to its library. SOOOOO excited!!! How FURIOUS } \\
\text { LOVE all began.... }\end{array}$ \\
\hline watching & Watchin netflix \\
\hline snafu & Streaming Netflix is being stupid again \\
\hline snafu & widespread panic!Netflix streaming is down!\#Netflix \#Panic \\
\hline complaint & RT @joemsf: widespread panic!Netflix streaming is down!\#Netflix \#Panic \\
\hline complaint & No,Netflix is not working for me on Android. \\
\hline complaint & Netflix turned the app off. Assholes. \\
\hline snafu & Damn Netflix is acting stupid right now \#Pissed \\
\hline snafu & Netflix is down. WUT!? :[ \\
\hline snafu & fucking netflix is taking forever to load. i just wanna watch dexter. darn. \\
\hline snafu & $\mathrm{Y}$ is Netflix tripping? Grrrr! \\
\hline complaint & $\begin{array}{l}\text { Dude. Seriously, what happened to the @Netflix app. It freakin’ blows now. @instant_netflix @netflix- } \\
\text { helps }\end{array}$ \\
\hline complaint & is anybody else's netflix being really slow? \\
\hline snafu & is anybody else's netflix being really slow? \\
\hline response & @geezymcgee naw just Netflix. \\
\hline snafu & @geezymcgee naw just Netflix. \\
\hline snafu & WTF? Netflix just crapper out on me? \\
\hline
\end{tabular}




\begin{tabular}{|c|c|}
\hline complaint & Fucking netflix!!! \\
\hline snafu & Pissed my fucking \#netflix not working \\
\hline snafu & @Netflix seems to be having trouble loading tonight. \\
\hline complaint & Netflix is giving me a fucking headache \\
\hline snafu & So netflix isnt workin? \\
\hline snafu & Why is Netflix on xbox fucked up? \\
\hline snafu & Having problems getting on @netflix sigh \\
\hline snafu & ahhh i hate you crack whore Netflix, once again ur down for the count \\
\hline complaint & \#netflix is buggin' \\
\hline snafu & \#netflix is buggin' \\
\hline snafu & Wth my netflix just broke \\
\hline complaint & Wtf my netflix is malfunctioning \\
\hline snafu & Wtf my netflix is malfunctioning \\
\hline snafu & @jujukoo NETFLIX??!?!? \\
\hline snafu & Damn Netflix. work!!! \\
\hline snafu & Netflix is down...smh... \\
\hline snafu & Netflix isn't working \#firstworldproblems \\
\hline snafu & Problems with Netflix instant on wii. So slow tonight. \\
\hline complaint & Dammit netflix \\
\hline snafu & Is \#Netflix down? \\
\hline snafu & about to get super \#pissed at fuckin Netflix \\
\hline complaint & @netflix @Netflixhelps Useless CUNTS! \\
\hline snafu & \#netflix is down :-( \\
\hline snafu & Fucking netflix isn’t working \\
\hline snafu & NETFLIX WHAT THE FUCK IS YOUR MAJOR MALFUNCTION? HUH?? \\
\hline happy & netflix is tha shittt \\
\hline complaint & Wtf \#Netflix, down again ?? \\
\hline snafu & Wtf \#Netflix, down again ?? \\
\hline snafu & Is @Netflix down? \\
\hline complaint & Sigh@netflix \\
\hline
\end{tabular}




\begin{tabular}{|c|c|}
\hline snafu & is anyone elses netflix actin up? \\
\hline snafu & Wtf y isn't netflix working \\
\hline snafu & NetFlix down on playstation BUST \\
\hline complaint & Man y the fuck aint my netflix workin smdh \\
\hline snafu & Man y the fuck aint my netflix workin smdh \\
\hline complaint & Netflix is pissin me off tonight \\
\hline complaint & Netflix down........bummer \\
\hline snafu & Netflix down........bummer \\
\hline complaint & Is \#Netflix broken? \\
\hline snafu & Is \#Netflix broken? \\
\hline complaint & \#NETFLIX WII FAIL!!! \\
\hline snafu & \#NETFLIX WII FAIL!!! \\
\hline complaint & Got damn netflix is fuckin up smh \\
\hline snafu & Got damn netflix is fuckin up smh \\
\hline complaint & Right! fucking pieces of shit, did this happen last sunday same time too @Goddard23 Fuck you netflix \\
\hline snafu & Right! fucking pieces of shit, did this happen last sunday same time too @Goddard23 Fuck you netflix \\
\hline snafu & @pitaman @netflix Yep. Netflix is messing up. \\
\hline complaint & @mommywantsvodka We're having probs too. Boo you Netflix. Boo. \\
\hline snafu & @mommywantsvodka We're having probs too. Boo you Netflix. Boo. \\
\hline snafu & Meh, come on, apparently \#netflix is having instant streaming outages. \\
\hline snafu & Netflix is fucking up tryna watch god damn prison break \\
\hline snafu & Fucking Netflix, fucking work!!!!! \\
\hline snafu & Damn it.......Netflix Streaming is down AGAIN!!!! \\
\hline snafu & Hey netflix quit fucking around I wanna watch a movie \\
\hline snafu & Sad thing though, @netflix is having troubles connecting. \\
\hline snafu & This fuckin netflix actin up \\
\hline complaint & WTF Netflix \#smh \\
\hline snafu & @L4Lyfe yeah.. netflix is just being gay \\
\hline snafu & Netflix is down. I'm hella pissed \\
\hline complaint & Grrrr @Netflix you're broken AGAIN! Wtf?? \\
\hline
\end{tabular}




\begin{tabular}{|c|c|}
\hline snafu & Grrrr @Netflix you're broken AGAIN! Wtf?? \\
\hline happy & Netflix + me $=$ happiness $; 3$ \\
\hline snafu & Grumble. Netflix on the xbox is down. Boo cloud :( \\
\hline snafu & This weather fucking up my netflix \\
\hline complaint & WTF, Netflix? AGAIN? \\
\hline snafu & WTF, Netflix? AGAIN? \\
\hline complaint & FUCK WHERE'D NETFLIX GO \\
\hline snafu & FUCK WHERE'D NETFLIX GO \\
\hline snafu & Netflix won't open. \#WhatTheFuck \#IMightAsWellBeDead \\
\hline complaint & Our neighbors WIFI is fuckin up. Im gettin pissed off. Niggas tryin to watch Netflix -_- \\
\hline complaint & Netflix is down. Dang. \\
\hline snafu & Netflix is down. Dang. \\
\hline complaint & Why netflix why \\
\hline snafu & Why netflix why \\
\hline snafu & @Netflix is down on my xbox smh. \\
\hline snafu & @netflix is dead on the roku, works on the laptop... \\
\hline snafu & Ugh, @Netflix is turning into such a fail. \\
\hline snafu & Netflix is acting stupid there goes my night -_-- \\
\hline snafu & "Netflix is currently unavailable. try again later." \#NetFlix \#FML \#DrWho \#WhyMe \#Sad @Netflix \\
\hline snafu & Netflix ROKU \#FAIL \\
\hline snafu & RT @Vann_Rich: Netflix temporarily down WTH :( / WTF?! \\
\hline complaint & noting pisses me off like slow internet connections messing up my netflix experience \\
\hline complaint & Netflix fuck \\
\hline complaint & These stupid ass netflix dvds don't let u skip past the previews smdh \#bullshit \\
\hline watching & This week on the \#Netflix line-up: Fair Game and I Love You Phillip Morris. \\
\hline happy & \#Netflix you've been gone for too long! \#welcome back \\
\hline neutral & shitty part about this last breakup: i got the cat, she got the netflix account. \#whattodonow.. \\
\hline watching & Watching Fullmetal Alchemist: Brotherhood on Netflix while we wait to get into the production set space \\
\hline happy & Uh oh \#glee is on \#netflix now \\
\hline neutral & Uh oh \#glee is on \#netflix now \\
\hline
\end{tabular}




\begin{tabular}{|c|c|}
\hline happy & $\begin{array}{l}\text { RT @robert3t: @silug @DirecTV_TiVoHave you tried Roku with Netflix/Amazon/Crackle/Hulu Plus - } \\
\text { it's like letting someone else TiVo for you. }\end{array}$ \\
\hline neutral & $\begin{array}{l}\text { RT @robert3t: @silug @DirecTV_TiVoHave you tried Roku with Netflix/Amazon/Crackle/Hulu Plus - } \\
\text { it's like letting someone else TiVo for you. }\end{array}$ \\
\hline watching & Watching 3rd Rock from the Sun marathon on Netflix. \\
\hline media & ok @MissGinaDarling... u need to bring your kinect back to my house now =P http://aol.it/fKVBe9 \\
\hline happy & Dear Netflix, I love you a lot. \\
\hline watching & $\begin{array}{l}\text { Watching @ToddGlass on Netflix. Had to follow him ASAP, never laughed so hard alone in the dark, } \\
\text { stoned, eating cake at } 5 \text { am... This week... }\end{array}$ \\
\hline happy & now time for some netflix $; 3$ \\
\hline watching & \#Watching 3rd Rock from the Sun marathon on Netflix, suck it BlockBuster... yeah I said it. \\
\hline watching & Watching Angus on Netflix instant. Huge h/t to @TedDouglass. Angus!! Love it or quit being my friend. \\
\hline happy & Thank god for netflix! Makes this 12 hour shift go by alot faster... \\
\hline watching & $\begin{array}{l}\text { SMH @ me taking this long to re-activate Netflix on my Xbox. was up all night watching foreign gangster } \\
\text { flicks/anime }\end{array}$ \\
\hline happy & @ YoungMykewut game r u playin ? \& . im watchin season 2 of jersey shore on netflix lol \\
\hline watching & 5am and \#nowwatching Stephen King's The Langoliers on netflix \\
\hline watching & I should be sleeping- not tweeting- but I'm watching Cheers on Netflix and it's addictive. \#ILoveSitcoms \\
\hline watching & I 100\% should go to bed RIGHT NOW. But, I'm up watching even more netflix! oh boy, oh boy. \\
\hline watching & Watching Glee on netflix music is dope story ia gay but chicks are dope \\
\hline happy & So I'm about to hit up netflix while I'm eating pudding out of a cup with merely my tongue ;D \\
\hline watching & So I'm about to hit up netflix while I'm eating pudding out of a cup with merely my tongue ;D \\
\hline happy & Just found the original twilight zone on Netflix. Awesome. \\
\hline watching & Guess I'll watch some Netflix \\
\hline media & $\begin{array}{l}\text { Stock Investing — Can Netflix Push Forward By Benefiting From Online Streaming Video? } \\
\text { http://dlvr.it/Nh7HZ }\end{array}$ \\
\hline media & $\begin{array}{l}\text { Stock Investing — Can Netflix Push Forward By Benefiting From Online Streaming Video? } \\
\text { http://dlvr.it/Nh7HT }\end{array}$ \\
\hline media & $\begin{array}{l}\text { Stock Investing - Can Netflix Push Forward By Benefiting From Online Streaming Video? } \\
\text { http://dlvr.it/Nh7HG }\end{array}$ \\
\hline
\end{tabular}




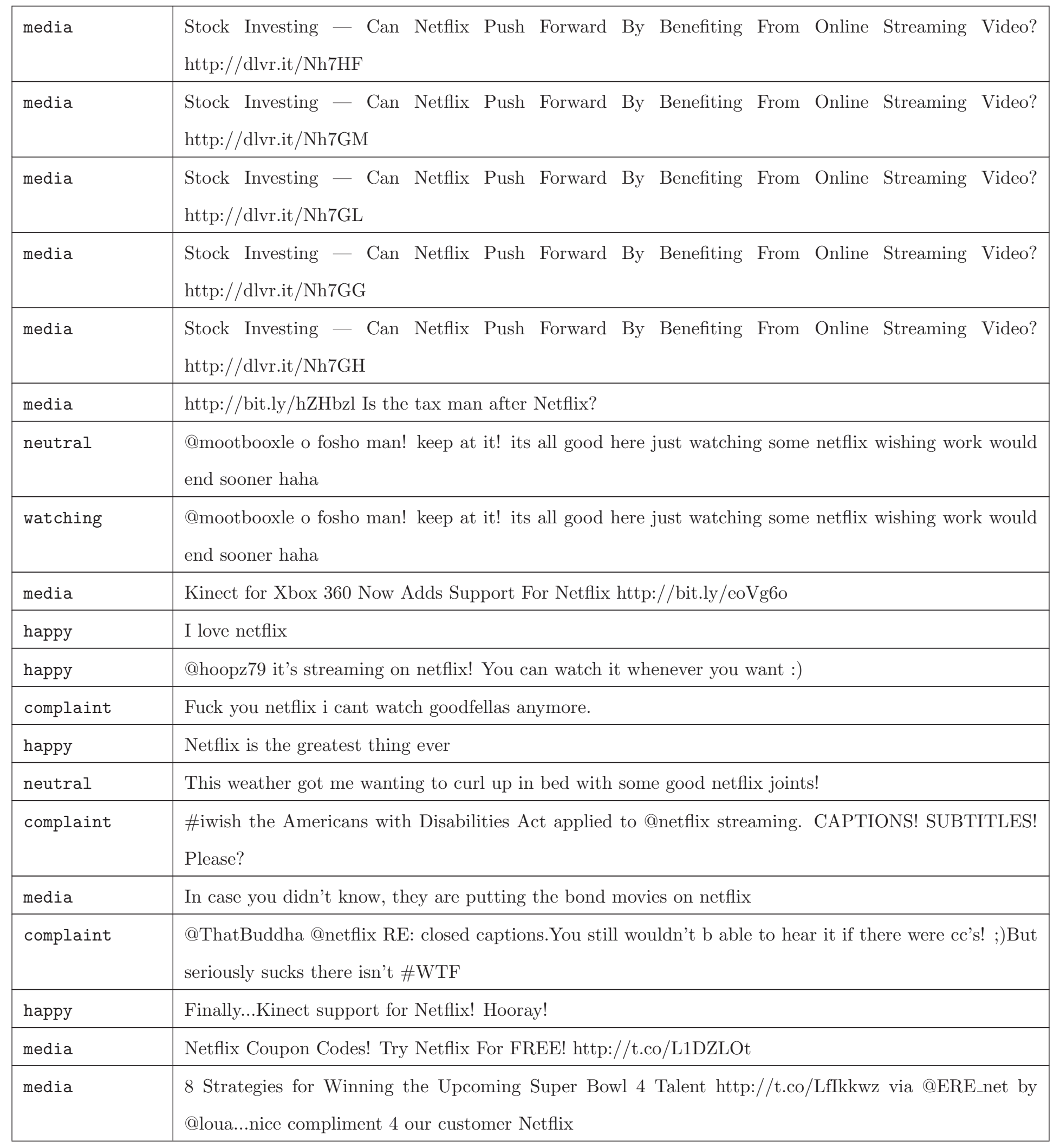




\begin{tabular}{|c|c|}
\hline response & @.JBoyd4 Yeah. Cause then Netflix will suggest some terribly similar movies. \#crap \\
\hline neutral & I need to remember to buy batteries for my wiimote. I haven't watched a Netflix movie in weeks. \\
\hline watching & Type bored watching netflix on my couch. \\
\hline watching & thank you \#netflix !! now watching \#heyarnold :) takes me back to better days lol \\
\hline complaint & $\begin{array}{l}\text { damn you @netflix . why cant you support @linux ? @hulu can. silverlight is garbage and there is no } \\
\text { work around. get with the times!!! }\end{array}$ \\
\hline watching & $\begin{array}{l}\text { Just started watching the devil may cry series on netflix, mad as fuk that I slept on this. You see it? } \\
\text { @Dreadhead1914 }\end{array}$ \\
\hline watching & I've watched almost every documentary on netflix \\
\hline neutral & $\begin{array}{l}\text { so today will consist of Dominos pizza \& netflix, while icing this ankle of mine .. ehh : complaint,RT } \\
\text { @FreshPres: Netflix is disrespectful for not having Coneheads On their site. }\end{array}$ \\
\hline happy & Netflix *thumbs up* \\
\hline watching & $\begin{array}{l}\text { Watching Capitalism: A Love Story on Netflix.....this movie is sooo relevant right now. Check it out if } \\
\text { you haven't seen it. }\end{array}$ \\
\hline complaint & $\begin{array}{l}\text { @ netflix has every old james bond flick but goldfinger, from russian with love and dr. no. ill take that as } \\
\text { a big "fuck you." }\end{array}$ \\
\hline watching & RT @PornStarPaul: netflix type of day* I'm watching nexflix now -_- \\
\hline happy & omg...all seasons of ghost whisperer\& scrubs on netflix...love it! \\
\hline complaint & $\begin{array}{l}\text { My sister must have put a Tyler Perry movie in my Netflix Queue.. Someone done lost they mind I see... } \\
\text { Fuck Tyler Perry }\end{array}$ \\
\hline watching & $\begin{array}{l}\text { My sister must have put a Tyler Perry movie in my Netflix Queue.. Someone done lost they mind I see... } \\
\text { Fuck Tyler Perry }\end{array}$ \\
\hline happy & Netflix to my rescue. ¡3 http://t.co/hrv4sS7 \\
\hline media & Netflix Kinect: Control Movies With Your Hands http://t.co/ILePgA5 Welcome to the future. \\
\hline happy & Still watching \#glee on \#netflix :D \\
\hline watching & Still watching \#glee on \#netflix :D \\
\hline neutral & $\begin{array}{l}\text { I like that they made the netflix trial commercials look like a match.com commercial. \#fillyourloneli- } \\
\text { nesswithmovies }\end{array}$ \\
\hline media & Panasonic DMP-BD85K : Enjoy The Netflix Feature http://is.gd/QQaOp3 \\
\hline
\end{tabular}




\begin{tabular}{|c|c|}
\hline complaint & $\begin{array}{l}\text { Fuck you netflix! You fucken piece of shit! I can’t even fucking sign up for my free month because I'm " } \\
\text { http://tl.gd/9sr2ps }\end{array}$ \\
\hline media & Xbox 360 Update Adds Kinect Support for Netflix - Maximum PC http://sns.mx/Jvc8y8 \#Xbox \\
\hline happy & $\begin{array}{l}\text { God I love \#Netflix. Not only do I get new movies to go with today's perfect movie-watching weather, I } \\
\text { get the bonus of mail presents, too. }\end{array}$ \\
\hline watching & Watching Inheritance on Netflix.. damn \\
\hline watching & Watching the other guys on netflix \\
\hline watching & Watching Family Guy on Netflix \\
\hline watching & Watching marmaduke on netflix \\
\hline watching & Watching the Cosby Show on Netflix with the kids. So funny! \\
\hline watching & watching naruto in japanese with Manik on Netflix. it's like I'm 10 again. with Junior \& Cindy. \\
\hline happy & The twilight zone is on Netflix my life is complete. \\
\hline complaint & $\begin{array}{l}\text { Hey @netflix - streaming is great, but would closed captions for the hearing impaired kill you? Give us } \\
\text { access to the subtitle options? }\end{array}$ \\
\hline complaint & Someone at Netflix is fucking up, who put All About the Benjamins under Suspenseful Movies? \\
\hline complaint & $\begin{array}{l}\text { @FENTONFALLON I like netflix but am sad that not EVERYTHING on there is instant. Sometimes I } \\
\text { don't want to wait for it to come in the mail! }\end{array}$ \\
\hline happy & Cosby Show on @netflix... FTw!!! \\
\hline happy & I love watching Netflix in my living room TV!!! \\
\hline happy & RT @AlexGoral: Netflix is the greatest thing ever \\
\hline happy & i love netflix's romance section \\
\hline happy & RT @AlexGoral: Netflix is the greatest thing ever \\
\hline watching & Watching The Last Song, on Netflix. :) \\
\hline watching & Watching \#stargate atlantis on netflix. \\
\hline watching & is watching The Other Guys\#My6http://vapr.me/2vf7 \\
\hline complaint & $\begin{array}{l}\text { They say in } 5 \text { years we'll b able } 2 \text { replace our own organs w our own cells, but why can't I instant } \\
\text { netflixThe Hand That Rocks the Cradle? }\end{array}$ \\
\hline complaint & $\begin{array}{l}\text { Why doesn't \#Netflix have more social aspects? Isn't going to/watching movies primarily a social } \\
\text { activity? }\end{array}$ \\
\hline media & \#netflix stream from PC http://bit.ly/cAjJ99@GetGlue \#BizarreFoods \\
\hline
\end{tabular}




\begin{tabular}{|c|c|}
\hline complaint & Netflix sucks! \\
\hline neutral & $\begin{array}{l}\text { contemplating what I should do till Portal } 2 \text { drops... 1. a short game } 2 \text {. work on music } 3 \text {. catch up on } \\
\text { netflix... maybe all three }\end{array}$ \\
\hline neutral & $\begin{array}{l}\text { @spookypastor this is going to be a wallet-draining summer at the movies. Thankfully, there's a } 42 \text { " TV } \\
\text { with surround at home...and Netflix. }\end{array}$ \\
\hline response & $\begin{array}{l}\text { @spookypastor this is going to be a wallet-draining summer at the movies. Thankfully, there's a } 42 \text { " TV } \\
\text { with surround at home...and Netflix. }\end{array}$ \\
\hline watching & Such an ugly day. Perfect for a Sons Of Anarchy marathon on Netflix i:) \\
\hline watching & Watching The Office on Netflix, doing Laundry, and slowly cleaning the house. \\
\hline response & $\begin{array}{l}\text { @Br0wn_Suga it's on Netflix. It's called city island. It's so funny. Fat girls cookin butt naked in the } \\
\text { kitchen live on the web cam. Lol }\end{array}$ \\
\hline neutral & $\begin{array}{l}\text { @ natheist Ok, I have a working silverlight install from http://go-mono.com/moonlight/ Can't test it } \\
\text { against netflix, mind trying it? }\end{array}$ \\
\hline happy & Thank God for Netflix! :D \\
\hline neutral & $\begin{array}{l}\text { Got an email from Netflix. Dvd which never arrived and I reported lost has been returned to them. Odd, } \\
\text { that. }\end{array}$ \\
\hline happy & Netflix biotxhh!??? \\
\hline watching & Gone to watch me some Netflix... \\
\hline response & $\begin{array}{l}\text { @DarkEyeSocket I saw it on Netflix "Instant Watch." It's never been officially released on DVD but you } \\
\text { may be able to find a bootleg on ebay }\end{array}$ \\
\hline watching & $\begin{array}{l}\text { @DarkEyeSocket I saw it on Netflix "Instant Watch." It's never been officially released on DVD but you } \\
\text { may be able to find a bootleg on ebay }\end{array}$ \\
\hline complaint & $\begin{array}{l}\text { \#Netflix is more like net flicker lately. Pretty sure it's been down at least half the time I've tried to use } \\
\text { it this month. }\end{array}$ \\
\hline snafu & $\begin{array}{l}\text { \#Netflix is more like net flicker lately. Pretty sure it's been down at least half the time I've tried to use } \\
\text { it this month. }\end{array}$ \\
\hline neutral & $\begin{array}{l}\text { Wwe has recently just posted over } 7 \text { new doucumentaries and wwe studios movies in netflix including } \\
\text { "the john cena experince" watch them now! }\end{array}$ \\
\hline watching & $\begin{array}{l}\text { Wwe has recently just posted over } 7 \text { new doucumentaries and wwe studios movies in netflix including } \\
\text { "the john cena experince" watch them now! }\end{array}$ \\
\hline
\end{tabular}




\begin{tabular}{|c|c|}
\hline neutral & Yay my first Netflix film is on its way; predicted arrival time is Monday. Woot. \\
\hline neutral & about to download this netflix free trial \\
\hline neutral & Just bought one month of Netflix. God help me. \\
\hline watching & I need recommendations for good movies to watch on Netflix!? \\
\hline media & $\begin{array}{l}\text { Netflix is looking for: Senior Software Engineer - Recommendation A... http://jobvite.com/m?3Mny3fwu } \\
\text { \#job }\end{array}$ \\
\hline neutral & Groundbreaking news: I have netflix!! \\
\hline response & $\begin{array}{l}\text { @_shalyn its hilarious i didnt even know what it was til my friend told me to check it out.. netflix has it } \\
\text { so that rocks. i was dying when }\end{array}$ \\
\hline neutral & $\begin{array}{l}\text { Friday night: got to cook } 3 \text { pizzas (food allergies!), figure out what movie to watch on Netflix, and do } \\
\text { some tatting and knitting. }\end{array}$ \\
\hline neutral & I love those random moments of netflix when you get hd for like ten seconds \\
\hline watching & Just turned on Netflix, watching Poetic Justice. I'm on Janet mode right now. \\
\hline neutral & $\begin{array}{l}\# \text { fe3 [Shelton ] Big data means algorithms will find the patterns (so humans don't have to).Amazon rec } \\
\text { and Netflix suggest everywhere. }\end{array}$ \\
\hline complaint & Watching material girls on youtube since ournetflix acting stupid \\
\hline snafu & Watching material girls on youtube since ournetflix acting stupid \\
\hline watching & $\begin{array}{l}\text { Watching Anastasia on @Netflix while baking blueberry oatmeal cookies. It's been a glorious Saturday } \\
\text { morning. }\end{array}$ \\
\hline neutral & $\begin{array}{l}\text { @JackkiJack I haven't watched buffy, but I'm always down for suggestions! I love love love netflix instant } \\
\text { cue! }\end{array}$ \\
\hline complaint & $\begin{array}{l}\text { I was told that all of Star Trek was coming to Netflix Instant. I have yet to see it. Someone needs to } \\
\text { answer some questions. \#khan!! }\end{array}$ \\
\hline complaint & It looks like Netflix took season one of Doctor Who off instant streaming, which is sad :( \\
\hline happy & Where would I be without my netflix!? \\
\hline watching & Watchin the old V miniseries on netflix, love it \\
\hline complaint & Yo Netflix! That's a real bitch move having season 1-2 and none of the rest of it. Fuck is up?! \\
\hline media & Netflix is looking for: Driver 3 - Lansing http://jobvite.com/m?3vny3fwd \#job \\
\hline happy & i have been watching invador zim im sooo excited i llove netflix i love it im start to watch every episode \\
\hline watching & i have been watching invador zim im sooo excited i llove netflix i love it im start to watch every episode \\
\hline
\end{tabular}




\begin{tabular}{|c|c|}
\hline watching & i think im just going to watch netflix movies all day... \\
\hline neutral & $\begin{array}{l}\text { Finished ssn } 1 \text { of Glee...Season two isn't on Netflix...so im prolly screwed for a while since my mom just } \\
\text { bought my the first ssn last night. }\end{array}$ \\
\hline happy & Pizza + Netflix + Photoshop = Saturday fun \\
\hline watching & let me see what I can find on Netflix today... \\
\hline watching & Yes! Thank God for Netflix now I can watch movies on my iPod. \\
\hline neutral & $\begin{array}{l}\text { Toy Story } 3 \text { is available on Netflix today. Our kids HAD to watch it, even though they've been } 100 \% \\
\text { uninterested our DVD copy since day } 1 .\end{array}$ \\
\hline neutral & i find the strangest movies to watch on Netflix. \\
\hline watching & i find the strangest movies to watch on Netflix. \\
\hline watching & Watching Glee season 1, the season I missed. Thank gosh Netflix!! (: \\
\hline media & "Rivals target Netflix"http://bit.ly/dRjwsZ \\
\hline complaint & damn theres nothing to watch on netflix \\
\hline watching & $\begin{array}{l}\text { On a scale of 1-10, if The Wire was a 10, then Sons of Anarchy is a 9.5.Just finished the first season on } \\
\text { Netflix.Holy shit. }\end{array}$ \\
\hline watching & Twin Peaks on Netflix Instant.... Holy shit yes \\
\hline watching & Black Dynamite is on Netflix Instant.For the love of all that is holy, watch it. Thank me later! \\
\hline happy & \#Netflix :) \\
\hline happy & SPRING BREAAKK !! i celebrate by getting a free month of netflix MOVIES!! \\
\hline neutral & $\begin{array}{l}\text { \#Netflix ?20 more people please I need } 20 \text { more people whoever didn't take this survey please do it } \\
\text { http://www.surveymonkey.com/s/MLKZ2BZ }\end{array}$ \\
\hline watching & perhaps it's time to stop watching 'my so called life' on netflix and be productive today. \\
\hline happy & Netflix!! ¡33 \\
\hline media & $\begin{array}{l}\text { Samsung 3D Wi-Fi Blu-ray Disc Player + } 3 \text { Month Netflix Membership! RV \$280! \#Win @savvycoupon- } \\
\text { mom http://bit.ly/fBBNBc \#GIVEAWAY }\end{array}$ \\
\hline watching & Holy crap!! Netflix instant queue has MS3TK!!! \\
\hline watching & Dead Alive is on Netflix Instant? it's the Lord of the Rings of ridiculous zombie movies! \\
\hline complaint & @justinswife40 I Love Lucy is on Netflix, but not for streaming. =( I love I Love Lucy! \\
\hline watching & $\begin{array}{l}\text { Trying to get on the treadmill while the littlest one sleeps and the other } 2 \text { watch \#iCarly.So glad it is } \\
\text { streaming on \#Netflix. }\end{array}$ \\
\hline
\end{tabular}




\begin{tabular}{|c|c|}
\hline media & $\begin{array}{l}\text { Wii } 2 \text { Details, Netflix Gets Kinect, Gears of War } 3 \text { Beta Live Stream Video - IGN } \\
\text { http://go.ign.com/hLL4AR via @IGN }\end{array}$ \\
\hline happy & netflix :) \\
\hline happy & Netflix;3 \\
\hline complaint & Nightmare on Elm Street...oh wait, netflix sux \\
\hline complaint & Damn netflix \\
\hline complaint & Wow Netflix! I hate you. \\
\hline complaint & I hate netflix. \\
\hline happy & NETFLIX \#love \#love \#love \\
\hline complaint & aye fuck netflix right now!!! \\
\hline complaint & $\begin{array}{l}\text { I'm disappointed with Netflix Canada's Matt Damon selection... So Mr Wahlberg will be saving the } \\
\text { night! }\end{array}$ \\
\hline complaint & $*_{\text {fucks wit Netflix* }}^{*}$ \\
\hline neutral & $*_{\text {fucks wit Netflix* }}^{*}$ \\
\hline neutral & Netflix $+?=?$ \\
\hline complaint & $\begin{array}{l}\text { Bummed no new episodes till 2012, but at least "Mad Men Will Be on Netflix Instant in July" } \\
\text { http://t.co/a3r0Rve }\end{array}$ \\
\hline complaint & Netflix's iPhone app won't show you disc only movie details - what kind of idiot UX is that?! \\
\hline complaint & Aughhhh, alright Netflix, you win tonight. I GIVE UPPPP. \\
\hline happy & Netflix :D \\
\hline watching & Watching "the creature from the black lagoon" on Netflix, holllaaa \\
\hline neutral & $\begin{array}{l}\text { Jeff teased me with Arthur on netflix yesterday... He put it on, got me all excited then turned it off. } \\
\text { Basically I need netflix. }\end{array}$ \\
\hline response & @dermhurl I don’t have netflix :( \\
\hline neutral & And on that note I'm going to watch Netflix \\
\hline happy & Netflix Saved My Night \\
\hline happy & Netflix is on point !....where have this been all my life \\
\hline watching & Watching Toy Story 3 on instant netflix. One of my faves! :D \\
\hline neutral & Netflix on PS3 \\
\hline watching & Watching Cheers on Netflix.j3 \\
\hline
\end{tabular}




\begin{tabular}{|c|c|}
\hline happy & Netflix saves Lives \\
\hline media & Control Netflix Movies Using Gestures or Voice With Kinect http://yhoo.it/eTy7rB \\
\hline complaint & I swear netflix be weak as $\mathrm{f}^{*} \mathrm{ck}$ sometimes \\
\hline happy & I love Netflix \\
\hline watching & $\begin{array}{l}\text { Just watched @thewaywegetby on Netflix. Such a moving and inspiring documentary. The best I have } \\
\text { seen in a while. PLEASE WATCH IT! }\end{array}$ \\
\hline complaint & I'm going to resuspend my Netflix account nothing is on here \\
\hline media & $\begin{array}{l}\text { Video killed the radio star, but will streaming TV online lead to death of the big media players? } \\
\text { http://bit.ly/gliaAX (via @guardiantech) }\end{array}$ \\
\hline happy & $\begin{array}{l}\text { I just convinced my brother to drop his DirectTV and get a wireless router, a roku HD and a netflix } \\
\text { subscription. }\end{array}$ \\
\hline happy & $\begin{array}{l}\text { I wanna spend like } 3 \text { weeks of my life sitting on the couch watching netflix and eating hot pockets. } \\
\text { \#Ginaboothe }\end{array}$ \\
\hline happy & So they have Arthur on Netflix now! When I get off work, its a done deal! \\
\hline refuse to rate & Forgot my headphones at home today. That rules out @netflix or my iPod for nap time. \#bummer \\
\hline refuse to rate & $\begin{array}{l}\text { @rannahshell But you watch netflix all the time. You need something fun that you don't normally get } \\
\text { to do. }\end{array}$ \\
\hline response & $\begin{array}{l}\text { @rannahshell But you watch netflix all the time. You need something fun that you don't normally get } \\
\text { to do. }\end{array}$ \\
\hline media & Daily SV News: Earnings train: First stop, Netflix http://om.ly/BSOVA \\
\hline media & Netflix posts 'buy' button but still no transactions http://cnet.co/hlF1GO \\
\hline neutral & Wat movie should I watch on netflix \\
\hline happy & $\begin{array}{l}\text { @ JuliaBlueEyes :( Don't feel that way, friend. Btw I just found happy tree friends on Netflix and it } \\
\text { reminded me of senior year! Lol }\end{array}$ \\
\hline response & $\begin{array}{l}\text { @ JuliaBlueEyes :( Don't feel that way, friend. Btw I just found happy tree friends on Netflix and it } \\
\text { reminded me of senior year! Lol }\end{array}$ \\
\hline media & $\begin{array}{l}\text { Updated: Netflix earns coming out next week. RT @GMSV: Earnings train: First stop, Netflix } \\
\text { http://bit.ly/hmxTQ1 \#tech \#siliconvalley }\end{array}$ \\
\hline happy & The Pixar Story is streaming on netflix :) \\
\hline media & \# Xbox 360 Online - Kinect-controlled Netflix Available Today On Xbox 360 http://bit.ly/hK1gxi \\
\hline
\end{tabular}




\begin{tabular}{|c|c|}
\hline happy & And my Netflix obsession begins... \\
\hline happy & $\begin{array}{l}\text { @kdhnews. Blockbuster Video...Do you go there.... I have Netflix and Vudu,wit those } 2 \text { I'm afraid I don't } \\
\text { go to Blockbuster stores. }\end{array}$ \\
\hline response & $\begin{array}{l}\text { @kdhnews. Blockbuster Video...Do you go there.... I have Netflix and Vudu,wit those } 2 \text { I'm afraid I don't } \\
\text { go to Blockbuster stores. }\end{array}$ \\
\hline complaint & $\begin{array}{l}\text { @netflix idea for the website, make rated movies searchable by genre and rating, for seeking friend } \\
\text { recommends among thousands of ratings }\end{array}$ \\
\hline happy & Why im in class watching netflix hahaha the life \#teamiphone4 \\
\hline watching & Why im in class watching netflix hahaha the life \#teamiphone4 \\
\hline neutral & About to watch a movie on netflix \\
\hline neutral & Netflix \\
\hline refuse to rate & @Str8_No_Chas3r lol true. I say hulu plus for the TV shows. Netflix for the movies \\
\hline happy & Netflix Instant has added all of the Larry Sanders Show- GOOD! \\
\hline happy & $\begin{array}{l}\text { Rocko's Modern Life is but one awesome show I remember growing up and I love how netflix has it to } \\
\text { stream. }\end{array}$ \\
\hline happy & I realize I'm super late on this, but LOST is the shit! Thank you Netflix. \\
\hline happy & I purposely look for the grossest movies on netflix just to get a good laugh \\
\hline happy & @RussianDollFace oh I watched so many of those the other day on netflix! \\
\hline response & @RussianDollFace oh I watched so many of those the other day on netflix! \\
\hline complaint & HOW SAD. REBA ISN'T ON INSTANT NETFLIX. THIS IS A SAD DAY IN BASEBALL. \\
\hline watching & watching a scrubs marathon on Netflix. @zachbraff and @donald_faison you guys are fricken hilarious! \\
\hline neutral & Watching my Netflix til my bae hit me bck - finta ignore \#2mof dumb a** . lol \\
\hline watching & Watching my Netflix til my bae hit me bck - finta ignore \#2mof dumb a**. lol \\
\hline media & Top News- Netflix posts 'buy' button but still no transactions http://adf.ly/1FTkk \\
\hline happy & @kdhnews We haven't rented DVDs in years.Netflix all the way. \\
\hline response & @kdhnews We haven't rented DVDs in years.Netflix all the way. \\
\hline media & $\begin{array}{l}\text { mSpot streams brand-new movies to iOS devices: A video-streaming service is aiming to beat Netflix } \\
\text { and Hulu at t... http://bit.ly/dR22nU }\end{array}$ \\
\hline watching & Watching 'Sneakers' on \#Netflix. This could be a great re-make. \\
\hline
\end{tabular}




\begin{tabular}{|c|c|}
\hline watching & $\begin{array}{l}\text { My room is a shit-show so while I'm cleaning I'm watching Clean House on @netflix which means I'm } \\
\text { just watching other people clean... }\end{array}$ \\
\hline watching & Hey Arnold on Netflix...Oh yeah! \\
\hline watching & Wild Thornberry's S1:E2, "Dinner with Darwin" on Netflix. :) \\
\hline watching & Watching Buffy and not feeling too well. Thank god it's on netflix. \\
\hline media & $\begin{array}{l}\text { MSpot Streams Brand-new Movies to IOS Devices: A video-streaming service is aiming to beat Netflix } \\
\text { and Hulu at t... http://bit.ly/dEzgeS }\end{array}$ \\
\hline media & $\begin{array}{l}\text { MSpot Streams Brand-new Movies to IOS Devices: A video-streaming service is aiming to beat Netflix } \\
\text { a... http://bit.ly/ftU9fs \#technology }\end{array}$ \\
\hline complaint & Biggest bogey in (recent) Television history: Cancelling Life on Mars (US). Thank you Netflix! \\
\hline watching & Biggest bogey in (recent) Television history: Cancelling Life on Mars (US). Thank you Netflix! \\
\hline refuse to rate & $\begin{array}{l}\text { RT @jpyun: ?? App Store? ??????? "?????" ???? "????+??"?? ?? ?? app?? 10-20? ????? ???? ?? 1??, } \\
\text { Netflix, ??? ?? ?? ???. http://goo.gl/htwtS }\end{array}$ \\
\hline happy & Luckily, have Netflix \& video games. That'll be nice. \\
\hline happy & $\begin{array}{l}\text { Watched the first episode of \#TwinPeaks last night with hubby.He was not impressed.But I loved it.Loving } \\
\text { \#Netflix. }\end{array}$ \\
\hline watching & $\begin{array}{l}\text { Watched the first episode of \#TwinPeaks last night with hubby.He was not impressed.But I loved it.Loving } \\
\text { \#Netflix. }\end{array}$ \\
\hline snafu & Netflix shawty \\
\hline happy & Netflix is the fucking BOMB!!!! \\
\hline complaint & Damn netflix \\
\hline media & Netflix to Become Largest Subscription Entertainment Business in U.S. http://j.mp/hdN3IA \\
\hline media & $\begin{array}{l}\text { Netflix to Become Largest Subscription Entertainment Business in U.S. http://j.mp/hdN3IA ????? ???? } \\
\text { ??? ???? ?? ??. ?? ??? 1?? ?? ??! }\end{array}$ \\
\hline complaint & Damn netflix. -.- \\
\hline snafu & Bored and Netflix is being stupid. \\
\hline complaint & OMG I HATE NETFLIX!!! \\
\hline complaint & Netflix \#wtf \\
\hline snafu & Netflix pissin me off!!! \\
\hline snafu & My fucken netflix fucken up. I'm tryna watch \#avatar:thelastairbender \\
\hline
\end{tabular}




\begin{tabular}{|c|c|}
\hline complaint & Fuck Netflix $i:[$ \\
\hline complaint & @reishka stupid netflix! \\
\hline snafu & whyy netflix actin so slowww! \\
\hline complaint & Netflix suck ass \\
\hline snafu & @krisfluck my stupid netflix instand keeps freezing on my tv....grrrrrrrrr \\
\hline snafu & Fukn Netflix just cut off. Lol \\
\hline snafu & Netflix pissin me off \\
\hline complaint & wtf \#netflix ?????? \\
\hline snafu & RIP Netflix.. \\
\hline complaint & Damn Netflix \\
\hline watching & Watching terrible netflix movies with brad. Hahahahahaha. \\
\hline snafu & yo wtf netflix?! \\
\hline snafu & Netflix????????????????????? \\
\hline snafu & Netflix???????????????????????????? \\
\hline watching & Watching Prison Break on Netflix. \\
\hline happy & HELL YES. netflix works again :) \\
\hline complaint & I hate you netflix \\
\hline snafu & NETFLIX WONT FUCKIN WORK . \\
\hline snafu & I'm mad my Netflix is being slow -_- \\
\hline complaint & Netflix=gay \\
\hline snafu & Netflix is being stupid, again \\
\hline snafu & SHIT netflix acting funny \\
\hline snafu & Netflix is \#lame \\
\hline complaint & I want dubbed, Netflix... fucking assholes. \\
\hline snafu & Netflix was being stupid today. Ugh \\
\hline snafu & Grrrrrr stupid PS3 won't let me stream Netflix. \\
\hline happy & ¡3 netflix \#addicted \\
\hline watching & watchin Netflix \\
\hline snafu & I hate when netflix fucks up shit is annoying \\
\hline media & TechCrunch? Netflix?30???????????????????????? http://dlvr.it/PhxTm \\
\hline
\end{tabular}




\begin{tabular}{|c|c|}
\hline snafu & Got my netflix fucked up \\
\hline snafu & Fuck netflix this bullshit \\
\hline complaint & smh, netflix ruins lives. \\
\hline complaint & Fuck \#NetFlix \\
\hline media & How Netflix Stole My Eyepatch \& I Stopped Stealing Movies http://tinyurl.com/3rgoqes \\
\hline refuse to rate & $\begin{array}{l}\text { Netflix (ya tienen casi } 23 \text { millones de usuarios) sube y el uso de Bittorrent baja en EEUU. Pasar lo mismo } \\
\text { por aqu? \#lodudo }\end{array}$ \\
\hline refuse to rate & Subieron la primera temporadade Glee a Netflix pero no me atrevo a verla \\
\hline refuse to rate & @burritosound estaba chebere, graciosa. Pero no pagues por verla maximo NETFLIX \\
\hline watching & \#Nowwatching: Black Snake Moan \#netflix \\
\hline refuse to rate & $\begin{array}{l}\text { @fernanhugo tal vez por eso Netflix vaya a abrir en Latinoamrica donde las derechos de cine se venden } \\
\text { para toda la region }\end{array}$ \\
\hline refuse to rate & Interesante artculo sobre \#Netflix. Muy interesante. http://www.asinorum.com/netflix/2699/ \\
\hline refuse to rate & $\begin{array}{l}\text { @manuxcristobal @fernanhugo existe la posibilidad de adquirir derechos no territorialmente y hacerlo } \\
\text { por lengua. Latinoamerica Netflix? }\end{array}$ \\
\hline refuse to rate & OMGGGGG Entrar a hulu, netflix y tal solo cambiando una DNS y funcionando sin proxis ni nada! \\
\hline complaint & i hate netflix \\
\hline refuse to rate & Cuando el servicio merece la pena se paga y Netflix es un ejemplo: \\
\hline refuse to rate & @Tavoteg nunca he usado netflix... \\
\hline happy & Netflix :) \\
\hline complaint & Fugg I hate how Netflix rewinds!! \\
\hline happy & Netflix :) \\
\hline refuse to rate & $\begin{array}{l}\text { Alguien llego a conocer el sitio argentino DVDinamic.com? Era el mismo modelo de negocio que net- } \\
\text { flix.com }\end{array}$ \\
\hline happy & Netflix ¡3 \\
\hline happy & Netflix ¡3 \\
\hline refuse to rate & uff, ahora xbox live tiene Hulu, sera mejor que Netflix, vamo a ver.... \\
\hline complaint & Netflix is being stupid right \\
\hline snafu & Netflix is being stupid right \\
\hline happy & Netflix :) \\
\hline
\end{tabular}




\begin{tabular}{|c|c|}
\hline refuse to rate & En momentos como este echo en falta Netflix. \\
\hline refuse to rate & Estan llegando buenas pelculas mexicanas a netflix \\
\hline happy & netflix;3 \\
\hline refuse to rate & "@tavoluna: Alguna buena pagina para ver pelis online?, sugieran" / netflix \\
\hline refuse to rate & Esta movie se ve interesante se llama MILF esta en netflix me fui \\
\hline complaint & Netflix movies REALLY fucking suck. \\
\hline happy & Netflix :) \\
\hline happy & \#Netflix :) \\
\hline happy & Netflix $; 3($ : \\
\hline happy & Yay Netflix (: \\
\hline happy & Seriously loveee netflix! (: \\
\hline happy & netflix :) \\
\hline happy & Netflix :) \\
\hline watching & Watching prison break on netflix \\
\hline happy & Netflix \\
\hline watching & watchin netflix \\
\hline watching & Watchin Netflix \\
\hline snafu & \#netflix is broken :( \\
\hline snafu & Netflix broken on TiVo? \\
\hline complaint & Huuhhh Netflix so fuckin slow \\
\hline refuse to rate & $\begin{array}{l}\text { @karito_villamar estoy viendo una en mi cuarto ya me conoces (netflix boy) jajaja la proxima vez si } \\
\text { veremos RIO jajaja }\end{array}$ \\
\hline watching & Watching lost on \#netflix \\
\hline refuse to rate & @jimenabauer / cual es?? dimela y la pido manana por netflix.... \\
\hline happy & Netflix is The Bomb (; \\
\hline refuse to rate & A pesar de no tener sueo me voy...buscare una pelicula mala en el netflix... \#SomniferoDeEmergencia \\
\hline watching & Watching Hey Aronald on Netflix \\
\hline complaint & Pissed netflix is down ... \#iCare \\
\hline snafu & Pissed netflix is down ... \#iCare \\
\hline happy & Netflix timeeee.! \\
\hline
\end{tabular}




\begin{tabular}{|c|c|}
\hline watching & Netflix timeeee.! \\
\hline happy & Netflix timeeee.! \\
\hline watching & Netflix timeeee.! \\
\hline happy & dear @netflix, i love you. i want to make sweet, sweet love to you. shhh, shhh it's ok..it's ok \\
\hline refuse to rate & $\begin{array}{l}\text { Woot Deals: End Of Month Sale! Insignia 1080p HD Blu-Ray Player WiFi Networking for Netflix/Cin- } \\
\text { emaNow/Pandora... f... http://bit.ly/lfPGbT }\end{array}$ \\
\hline watching & Watching Zach Galiafianakis standup on Netflix. Hilarious shit! \\
\hline happy & netflix is amazinggg(: \\
\hline happy & @lopedope yeeeeee netflix! \\
\hline happy & Netflix, I love you, even tho I hardly use you . \\
\hline happy & netflix is awesomeness...\#NW Friday \\
\hline happy & netflix is the shiiiiiiiiit \\
\hline refuse to rate & \#Netflix - Isso vai ser a minha salvao e por 12 dolares ao mes... \\
\hline happy & Man Netflix is awesome. \\
\hline happy & I love netflix.. \\
\hline refuse to rate & $\begin{array}{l}\text { Watch Hulu, Netflix, Comedy Central and More on Your Android Phone.: PlayOn can now stream... } \\
\text { http://goo.gl/fb/8XHG5 }\end{array}$ \\
\hline refuse to rate & $\begin{array}{l}\text { Guy and Madeline on a Park Bench: Black-and-white verit meets the charm of the classic Hollywood } \\
\text { musical in wri... http://bit.ly/ilCSTV }\end{array}$ \\
\hline watching & Watching Easy A on netflix :D \\
\hline refuse to rate & $\begin{array}{l}\text { @phroc Comparto } 100 \% \text { tu punto de vista con Spotify, y si sale el rumor de que pondrn pelculas y sale } \\
\text { antes que Netflix, tambin caer. }\end{array}$ \\
\hline watching & Just watched Joneses on Netflix. Awesome movie. \\
\hline watching & now im watching degrassi on \#netflix \\
\hline complaint & @VIIXXIVXCI Hmmmm I hate NetFlix \\
\hline refuse to rate & "@YungBDaInfamous: About To Watch Some Netflix!! Later \#TweetHeads" me too \\
\hline refuse to rate & $\begin{array}{l}\text { FYI: Netflix Knocks Comcast Off Its Throne - By: Brittney Wilson (Senior Editor)Over } 7 \% \text { of... } \\
\text { http://tinyurl.com/6b5pm5x \#MRT }\end{array}$ \\
\hline refuse to rate & $\begin{array}{l}\text { Y sorprendido al leer que stos mismos que se quejan del cambio en Spotify han bendecido en algn momento } \\
\text { el modelo de Netflix... \#spotify }\end{array}$ \\
\hline
\end{tabular}




\begin{tabular}{|c|c|}
\hline watching & watching this Anime called "Claymore" on Netflix - its really good lol \\
\hline watching & Watching the Pixar Story on Netflix \\
\hline refuse to rate & What the Music Business Can Learn From Netflix's Success http://bit.ly/grqcpL \\
\hline watching & Guess ill watch netflix \\
\hline refuse to rate & @lalai e a tv da sala netflix ready, mas quem diz que eu acho o modem? \\
\hline media & $\begin{array}{l}\text { Terrestrial Radio Needs to Embrace Its Online Future: Just as Netflix has admitted that TV Everywhere } \\
\text { provides a... http://bit.ly/mydSh1 }\end{array}$ \\
\hline media & "we want engineering teams to be used to a constant level of failure in the cloud" $i ¿$ http://bit.ly/mQGZkx \\
\hline media & Lessons Netflix Learned from the AWS Outage http://zite.to/k8ABGX via @Ziteapp \\
\hline media & $\begin{array}{l}\text { Netflix reflects on the AWS outage - http://tinyurl.com/3fl2566 (they are hosted on Amazon but were } \\
\text { not affected) http://bit.ly/k3sc7c }\end{array}$ \\
\hline media & $\begin{array}{l}\text { Terrestrial Radio Needs to Embrace Its Online Future: Just as Netflix has admitted that TV Everywhere } \\
\text { provides a... http://bit.ly/mAnsRS }\end{array}$ \\
\hline media & $\begin{array}{l}\text { Terrestrial Radio Needs to Embrace Its Online Future: Just as Netflix has admitted that TV Everywhere } \\
\text { provides a... http://bit.ly/mAnsRS }\end{array}$ \\
\hline media & Netflix, I love you, but you're big enough to make an iPad app that doesn't suck: http://t.co/sIKwZdT \\
\hline refuse to rate & $\begin{array}{l}\text { RT @mrgelk: ACTUALIZADO: Spotify NO se mete en el negocio de Streaming de Video - Talfin.net } \\
\text { http://t.co/umGks7M via @talfin }\end{array}$ \\
\hline media & $\begin{array}{l}\text { Netflix Said to Spend } \$ 1 \text { Billion in } 2011 \text { on Streaming Content http://t.co/tyDcuss via @technobuffalo } \\
\text { \#TechnoBuffalo }\end{array}$ \\
\hline watching & Watching Flashpoint on @netflix. Pretty much chilling out all day. Don’t have anything to do! :D \\
\hline happy & Yes Netflix thank you for having this. \#deadalive \\
\hline media & Lessons Netflix Learned from the AWS Outage http://j.mp/mQGZkx \\
\hline refuse to rate & $\begin{array}{l}\text { RT @myrealitytech: FYI: Netflix Knocks Comcast Off Its Throne - By: Brittney Wilson (Senior Edi- } \\
\text { tor)Over } 7 \% \text { of... http://tinyurl.com/6b5pm5x \#MRT }\end{array}$ \\
\hline media & $\begin{array}{l}\text { The discussion on the closed caption class action lawsuit against Netflix is still going strong at Roku } \\
\text { forum http://bit.ly/hiMKIy }\end{array}$ \\
\hline media & $\begin{array}{l}\text { Can Netflix Kill Illegal Downloads?: Analysis: TorrentFreak suggests legal movie streaming will reduce } \\
\text { the illeg... http://bit.ly/luY8s1 }\end{array}$ \\
\hline
\end{tabular}




\begin{tabular}{|c|c|}
\hline media & $\begin{array}{l}\text { Music Downloads: Can Netflix Kill Illegal Downloads?: You still can’t get good PC games free on Netflix, } \\
\text { or anyw... http://bit.ly/itg7mC }\end{array}$ \\
\hline complaint & RT @GreysonsGirl: I'm SO MAD A NETFLIX! I HOPE IT GOES BANKRUPT! \\
\hline complaint & I'm SO MAD A NETFLIX! I HOPE IT GOES BANKRUPT! \\
\hline watching & Gonna watch more netflix $\mathrm{x}$ ) \\
\hline media & Pretty interesting read - Lessons Netflix Learned from the AWS Outage http://zite.to/k8ABGX \\
\hline media & Can Netflix Kill Illegal Downloads? http://goo.gl/fb/pO1j0 \\
\hline media & Netflix releases a ton of fine titles to Instant today. Happy Streaming! http://fb.me/QdMat6iy \\
\hline complaint & RT @Lexandrahorn- Dear Netflix Instant Watch, Could you for once work on the weekend? \\
\hline snafu & RT @Lexandrahorn- Dear Netflix Instant Watch, Could you for once work on the weekend? \\
\hline refuse to rate & $\begin{array}{l}\text { this show is SICK! You gotta \#Netflix it and watch season one if you haven't, season two } \\
\text { tonight!"@NoReservations: TREME tonight!" }\end{array}$ \\
\hline media & Can Netflix Kill Illegal Downloads? - PCWorld http://bit.ly/jMeQxK \\
\hline refuse to rate & $\begin{array}{l}\text { Verizon document suggests LG Revolution will have Netflix pre-installed: We didn't exactly need any } \\
\text { more evidenc... http://bit.ly/mvZgXV }\end{array}$ \\
\hline snafu & NETFLIX WTF IS YOUR PROBLEM. STOP RETRIEVING. \\
\hline media & $\begin{array}{l}\text { RT @feedfliks: Netflix releases a ton of fine titles to Instant today. Happy Streaming! } \\
\text { http://fb.me/QdMat6iy }\end{array}$ \\
\hline refuse to rate & RT @ConanObrien: If I'm ever a ghost, I hope the person I haunt has Netflix. \\
\hline media & Can Netflix Kill Illegal Downloads? http://bit.ly/luY8s1 \\
\hline refuse to rate & $\begin{array}{l}\text { roku dlna-Viewsonic NexTV VMP75 1080p Network Media Player - Stream Netflix, Internet ra- } \\
\text { dios/videos \& access to ... http://bit.ly/moPiVn }\end{array}$ \\
\hline refuse to rate & $\begin{array}{l}\text { roku dlna-Viewsonic NexTV VMP75 1080p Network Media Player - Stream Netflix, Internet ra- } \\
\text { dios/videos \& access to ... http://bit.ly/moPiVn }\end{array}$ \\
\hline snafu & my Netflix acct is down... * stabs self in stomach* \#dead \\
\hline media & How to Trade Apple, Baidu, Netflix-and Win Big http://goo.gl/fb/ePPxj \\
\hline media & $\begin{array}{l}\text { RT @feedfliks: Netflix releases a ton of fine titles to Instant today. Happy Streaming! } \\
\text { http://fb.me/QdMat6iy }\end{array}$ \\
\hline media & $\begin{array}{l}\text { Lotsa nothing...zzzz..RT @feedfliks: Netflix releases a ton of fine titles to Instant today. Happy Streaming! } \\
\text { http://fb.me/QdMat6iy }\end{array}$ \\
\hline
\end{tabular}




\begin{tabular}{|c|c|}
\hline watching & Watchin this big foot show on netflix.big foot is real \\
\hline media & $\begin{array}{l}\text { Syndication is dead. NETFLIX is the ultimate re-run channel. Newest additions to Instant View here - } \\
\text { http://fb.me/QdMat6iy (via @ProgGrrl) }\end{array}$ \\
\hline media & $\begin{array}{l}\text { NETFLIX MANAGEMENT SECRETS: CEO Reed Hasting's Presentation On A Culture Of Freedom } \\
\text { And Responsibility http://read.bi/g9yj6D }\end{array}$ \\
\hline happy & netflix netflix netflix netflix ;3 ;D \\
\hline happy & I absolutely love @netflix ... where else can I find so many bad zombie movies to watch? :) \\
\hline media & $\begin{array}{l}\text { RT @feedfliks: Netflix releases a ton of fine titles to Instant today. Happy Streaming! } \\
\text { http://fb.me/QdMat6iy }\end{array}$ \\
\hline happy & @lauramv23 netflix saved the day \\
\hline media & $\begin{array}{l}\text { RT @MarkRaganCEO: How successful companies like@Ford and @Netflix reframe difficult situations } \\
\text { http://bit.ly/mTWQ7e }\end{array}$ \\
\hline happy & @GHFans Netflix! :) \\
\hline refuse to rate & $\begin{array}{l}\text { Photo: The Funhouse, dir. Tobe Hooper (1981) Now Streaming on Netflix..... } \\
\text { http://tumblr.com/xzl2cdnrgi }\end{array}$ \\
\hline refuse to rate & Netflix demuestra la rentabilidad del alquiler de vdeos en Internet http://awe.sm/5IcC8 \\
\hline watching & We're watching it now RT @TheBuie HIGHLANDER ON NETFLIX....! CHEA!! \\
\hline media & $\begin{array}{l}\text { Lots of good stuff! RT @feedfliks: Netflix releases a ton of fine titles to Instant today. Happy Streaming! } \\
\text { http://fb.me/QdMat6iy }\end{array}$ \\
\hline media & $\begin{array}{l}\text { RT @feedfliks: Netflix releases a ton of fine titles to Instant today. Happy Streaming! } \\
\text { http://fb.me/QdMat6iy }\end{array}$ \\
\hline complaint & @evilcrash9@axnollouse Can’t see it, Netflix hates my phone/can’t remember my account info \\
\hline refuse to rate & @settern So that's 2 Sorkin series (also Studio 60). If only West Wing would come to Netflix Instant. \\
\hline media & $\begin{array}{l}\text { www.ArcaVir.asia Can Netflix Kill Illegal Downloads?: Analysis: TorrentFreak suggests legal... } \\
\text { http://bit.ly/j6qLm2 \#security \#antivirus }\end{array}$ \\
\hline media & $\begin{array}{l}\text { RT @feedfliks: Netflix releases a ton of fine titles to Instant today. Happy Streaming! } \\
\text { http://fb.me/QdMat6iy }\end{array}$ \\
\hline media & $\begin{array}{l}\text { Yes! RT @feedfliks: Netflix releases a ton of fine titles to Instant today. Happy Streaming! } \\
\text { http://bit.ly/mEvOlH }\end{array}$ \\
\hline media & Netflix's chief: No plan to create Armageddon with pay TV providers http://bit.ly/loArMh \#netflix \\
\hline
\end{tabular}




\begin{tabular}{|l|l|}
\hline neutral & Netflix till work \\
\hline snafu & Netflix on my phone isn't working.... guess I'm napping in the library 'til 3 "/ \\
\hline watching & Watchibg dragon tiger gate on netflixM \\
\hline happy & Oh and btw I am really diggin' Netflix....love streaming those movies!! \\
\hline complaint & I HATE YOU AGAIN NETFLIX!! \\
\hline refuse to rate & $\begin{array}{l}\text { @parra Bueno... es gratis en algunos casso con publicidad. En otros son suscripcion. Similar a Netflix } \\
\text { (aunque yo prefiero Netflix XD) }\end{array}$ \\
\hline happy & Netflix? :) \\
\hline
\end{tabular}

